\title{
Optimal Location of Advance Warning for Mandatory Lane Change near a Two-Lane Highway Off-ramp
}

\author{
Siyuan Gong ${ }^{1}$, Lili Du
}

\begin{abstract}
Improper mandatory lane change (MLC) maneuvers in the vicinity of highway off-ramp will jeopardize traffic efficiency and safety. Providing an advance warning for lane change necessity is one of the efficient methods to perform systematic lane change management, which encourages smooth MLC maneuvers occurring at proper locations to mitigate the negative effects of MLC maneuvers on traffic flow nearby off-ramp. However, the state of the art indicates the lack of rigorous methods to optimally locate this advance warning so that the maximum benefit can be obtained. This research is motivated to address this gap. Specifically, the proposed approach considers that the area downstream of the advance warning includes two zones: (i) the green zone whose traffic ensures safe and smooth lane changes without speed deceleration (S-MLC); the start point of the green zone corresponding to the location of the advance warning; (ii) the yellow zone whose traffic leads to rush lane change maneuvers with speed deceleration (D-MLC). An optimization model is proposed to search for the optimal green and yellow zones. Traffic flow theory such as Greenshield model and shock wave analysis are used to analyze the impacts of the S-MLC and D-MLC maneuvers on the traffic delay. A grid search algorithm is applied to solve the optimization model. Numerical experiments conducted on the simulation model developed in Paramics 6.9.3 indicate that the proposed optimization model can identify the optimal location to set the advance MLC warning nearby an off-ramp so that the traffic delay resulting from lane change maneuvers is minimized, and the corresponding capacity drop and traffic oscillation can be efficiently mitigated. Moreover, the experiments validated the consistency of the green and yellow zones obtained in the simulation traffic flow and from the optimization model for a given optimally located MLC advance warning under various traffic regimes. The proposed approach can be implemented by roadside mobile warning facility or on-board GPS for human-driven vehicles, or embedded into lane change aid systems to serve connected and automated vehicles. Thus it will greatly contribute to both literature and engineering practice in lane change management.
\end{abstract}

Keywords: Mandatory lane change; Optimal lane change zones; Time delay; Shock wave

\footnotetext{
${ }^{1}$ Department of Civil, Architectural and Environmental Engineering, Illinois Institute of Technology, 3201 South Dearborn Street, Chicago, Illinois 60616 U.S.A., Email: sgong1@ hawk.iit.edu

${ }^{2}$ Corresponding author, Department of Civil, Architectural and Environmental Engineering, Illinois Institute of Technology, 3201 South Dearborn Street, Chicago, Illinois 60616 U.S.A., Email: ldu3@iit.edu
} 


\section{Introduction}

Lane change decision represents the tactical driving decision in a trip. It determines which lane the vehicle will move on in the near future. According to the circumstantial factors that trigger the intention of a lane-change, drivers' lane-change behaviors can be mainly classified into two types. (i) Discretionary lane change (DLC), which intends to improve his/her driving conditions. It is not required and usually happens according to drivers' habits. (ii) Mandatory lane change (MLC), which is forced because of unavoidable conditions, such as the end of a merging lane, and exit of off-ramp (Ahmed 1999; Halati et al 1997; FHWA 1998). Clearly, MLC is more trackable than DLC from a system point of view. This study thus is interested in developing strategic lane change guidance for MLC at off-ramp. The proposed research is motivated by several motivations below.

First of all, many existing studies have indicated that traffic congestion often happens at the diverge bottleneck due to low capacity at off-ramp lane. This congestion will propagate to upstream branch and blocks traffic flow further (Daganzo, 1999; Munoz and Daganzo, 2002; Cassidy et al., 2002; Jin and Zhang, 2005; Jin, 2009; Ahn et al., 2010). Moreover, recent research recognized that lane change maneuver is one of the main triggers of negative effect nearby off-ramp. For example, Leclercq et al. (2007) validated that relaxation phenomenon ${ }^{3}$ resulting from lane change maneuvers nearby on-ramp and off-ramp will cause traffic delay to surrounding vehicles, which will make a driver (a lane-changer or a follower) become more aggressive after this relaxation phenomenon (Zheng et al. 2013). Jin (2010, 2013) captured the capacity drop and jam densities, which are closely related to lane change maneuvers nearby both on-ramp and off-ramp. More generally, both researchers and engineers have noticed that improper lane change maneuvers will disrupt traffic streams, induce traffic oscillation and traffic breakdown, and even cause traffic congestion and accidents (Jula et al., 2000; Mauch and Cassidy, 2004; Laval and Daganzo, 2006; Ahn and Cassidy, 2007, Li and Ouyang, 2011, Zheng, 2014). Therefore, strategic guidance and management for lane change maneuvers around off-ramp are needed to promote both lane change safety and traffic efficiency, which thus motivates the proposed study.

In recent years, companying with the development of advanced sensing and communication techniques, a plenty of on-board lane change assistant systems such as collision avoidance system and automotive lane change aid (Talmadge et al., 2000; Vahidi and Eskandarian, 2003; Schofield, 2005; Ardelt et al., 2012; Park, 2012) have been developed. These systems are able to provide warning information about nearby vehicles' movement or location to improve the safety of a lane change maneuver once it starts. But they still suffer from avoiding rush lane change maneuvers due to two shortcomings. First, they cannot inform vehicles when and where to start the lane change intention, which is one of the key components to affect lane change decision and behavior (Choudhury, 2002). Hurry lane change maneuvers will happen more often if a lane change starts closer to an off-ramp (Hou et al, 2012). The existing on-board lane change aid systems usually rely on individual drivers' experience to decide when and where to start the lane change intention, thus the optimality even the properness cannot be guaranteed since they may not be able to accurately aware the local traffic condition. Second, they cannot sustain traffic efficiency since the effect of a lane change maneuver on traffic flow is not considered in these lane change aid systems. Thus, more comprehensive lane change guidance is needed.

Current lane change warning/management usually applies roadside advance warning. They will provide advance warning for off-ramp, lane merge, and lane division at a fixed point, such as " $1 / 2$ mile for Exit 41A" (FHWA, 2009). It is expected that vehicles will start lane change intention if needed once they see these warnings. Since the fixed signpost does not count traffic condition variation, it is usually unknown/uncertain to both traffic managers and drivers where is the best spot to locate the advance warning, which triggers the lane change intention so that we can obtain the maximum benefit, especially for new drivers or drivers who are not familiar with the trips. As a result, improper lane change behavior happens frequently in the vicinity of an off-ramp.

This negative effect of MLC maneuvers around work zone obtained more attentions than highway off-ramp in literature. Quite a few studies (Tarko et al., 1999; Mccoy and Pesti, 2001; Michigan DOT, 2004; Radwan, Zaidi, Harb 2011) proposed lane change management strategies around work zones, including early merge, late merge, and dynamic merge strategies. Engineering experiments indicated

\footnotetext{
3 Relaxation phenomenon: Lane changers will accept very short spacing as they enter the target lane and then "relax" to a more comfortable spacing (Laval and Leclercq, 2008).
} 
that early merge, which suggests lane merges at a location further away from a work zone, is effective under light traffic condition; late merge, which suggests lane merges at a location closer to a work zone, will minimize the queue length resulting from lane closure at work zone under congested traffic condition; and dynamic merge proposes a technique which alternatively uses early and late merge according to traffic flow condition. However, most of the proposed work applies engineering experiences rather than rigorous methods to determine the location of the advance warning for either early merge or late merge strategy. Thus, the best benefits of the systematic lane change management strategies may not be obtained. In addition, the proposed strategies for work zones cannot be directly used to manage the MLC maneuvers nearby the area of a lane division or off-ramp since they cause traffic flow variations different to the situation around work zones (Jin, 2009; Ahn et al., 2010; Radwan, Zaidi, Harb, 2011). The state of the art indicates that we are still lack of rigorous methods to guide MLC maneuvers nearby highway off-ramp, even though real-time traffic condition can be well detected.

Motivated by the above views, this study focuses on guiding MLC maneuvers in the vicinity of offramp on a two-lane highway segment with one entrance and one exit. All lane change maneuvers discussed hereafter in this paper imply MLC maneuvers without a particular specification. Our simulation experiments (see Figure 8) show that neither the advance warning for the lane change is given too early nor too late can result in minimum time delay, given all the vehicles need to exit at the off-ramp follow the warning to start their lane change intentions. This study thus seeks to locate an optimal spatial lane change zone for vehicles to start and then perform lane change maneuvers upstream to the exit so that enough safe lane change opportunities are sustained for individual vehicles, while traffic delay resulting from lane change maneuvers is minimized.

More exactly, the proposed approach separates the downstream area of the advance warning into two zones: (i) the green zone (immediate downstream of the advance warning) whose traffic ensures safe and smooth lane change without speed deceleration (S-MLC); (ii) The yellow zone (immediate downstream to the end of the green zone) whose traffic leads to rush lane change maneuvers with speed deceleration (D-MLC), which usually happens in an area close to the off-ramp with heavy traffic. The start point of the green zone corresponds to the optimal location to set the advance warning which starts a MLC intention to exit at the off-ramp. Thus, finding the optimal location to set the advance warning is equally to identify the corresponding optimal green and yellow zones downstream of the advance warning. This study proposes an optimization model combined with theoretic traffic flow analyses such as shock wave theories and macroscopic traffic flow models to identify the optimal green and yellow zones with given entering traffic flow data at the entrance of the highway segment. Considering only two variables (the green and yellow zones) are involved, a grid research algorithm is applied to search the optimal solution. The numerical experiments based on a simulation model in Paramics 6.9.3 are conducted to validate the applicability of the proposed approaches under various traffic regimes. The experimental results indicate that the proposed optimization model can successfully find the optimal green and yellow zones, thus optimally locate an advance warning so that the traffic delay resulting from the lane change maneuvers is minimized. Moreover, the experiments validated the consistency of the green and yellow zones obtained in the simulation traffic flow and from the optimization model under various traffic regimes. Our experiments also noticed that placing the advance warning at the optimal location will efficiently mitigate the capacity drop and traffic oscillation even though they are not explicitly involved in the development of our mathematical models.

To the best of our knowledge, this research represents one of the first efforts in literature, which develop rigorous mathematical models to search for the optimal location for putting the advance lane change warning and the corresponding optimal lane change zones. From the practice point of view, the proposed approaches can be implemented by roadside mobile warning facilities, or onboard GPS to provide guidance for human-driven vehicles. Or it can be integrated into lane change aid systems to serve connected and automated vehicles. Thus, the proposed research will greatly contribute to both literature and engineering practice in lane change management.

The proposed work is organized by the following structure. Section 1 introduces research background, motivations and then overviews the proposed research. Section 2 formulates the proposed research problem and defines the green and yellow lane change zones, related variables, parameters, and assumptions. Section 3 proposes the optimization model combined with traffic analyses to find the optimal lane change zones, which minimize the traffic flow time delay and protect enough smooth lane change opportunities. The proposed approaches are validated by numerical experiments given in 
Section 4. Section 5 summarizes the proposed work and proposes the future work rooted from this research.

\section{Problem Formulation}

This paper studies a two-lane highway segment with one entrance and one exit where an off-ramp is located on the right side. Figure 1 below provides an example. There are a number of vehicles on the Lane 2 need to perform MLC to Lane 1 so that they can exit the highway at the off-ramp. An advance warning will be given to start vehicles' lane change intention upstream to the off-ramp so that he/she can have enough time to find a safe spot do the lane change. This study separates the area downstream of the advance warning into two zones. The area with a length $L_{g}$ is referred to as the green zone, if its traffic condition ensures a smooth MLC without significantly interrupting traffic flow. Following the green zone, the area with length $L_{y}$ is referred to as the yellow zone, if its traffic condition leads to a MLC maneuver often with speed deceleration, which may cause significant traffic delay. It is expected that vehicles will start their lane change intentions once they obtain the advance warning, and will most likely complete their lane change maneuvers smoothly in the suggested zone. Correspondingly, the traffic delay resulting from the MLC maneuvers is minimized. It is noticed that the location of the advancing warning is affected by traffic condition as well as the MLC maneuvers in the green and yellow zones. On the other hand, the distribution of the green and yellow zones are affected by the location of the advance warning.

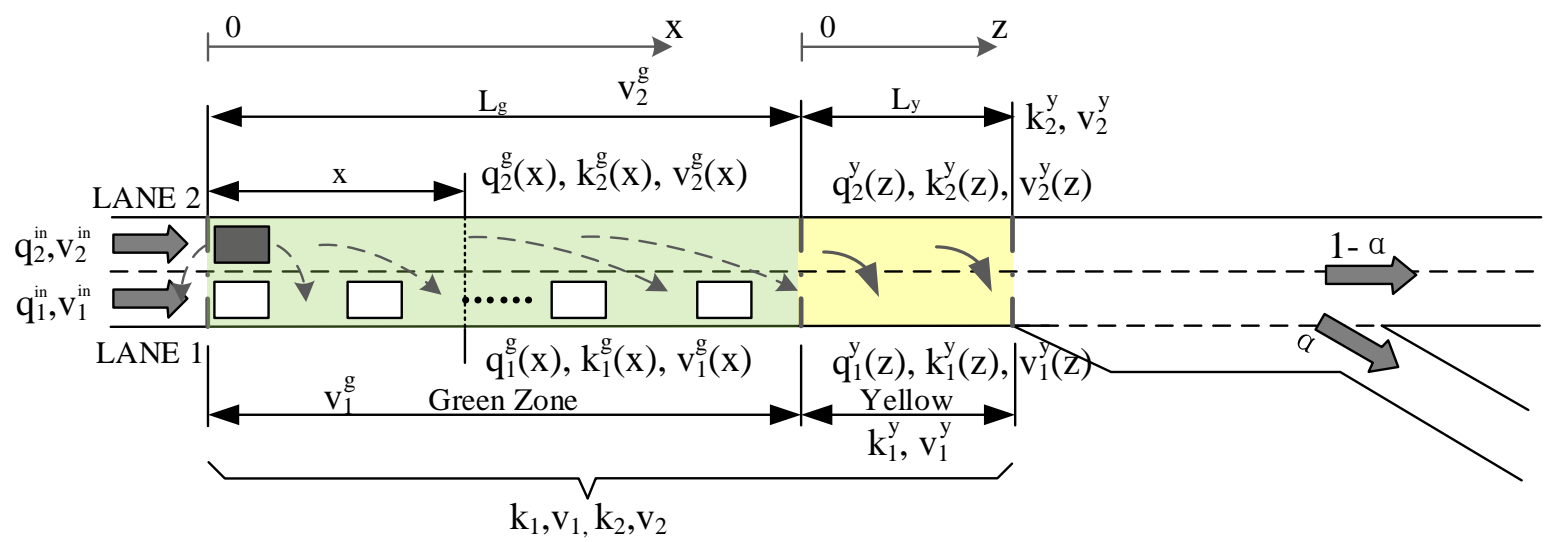

Figure 1. An example of a two-lane highway segment with an off-ramp

To conduct the proposed research, this study considers the traffic data including traffic flow and space mean speed data are available at the entrance of the highway segment, which are denoted as $q_{1}^{\text {in }}$ and $q_{2}^{i n}, v_{1}^{i n}$ and $v_{2}^{i n}$. According to traffic flow identity in traffic flow theory (Gerlough and Huber, 1975), we have the traffic density around the entrance, $k_{1}^{i n}=q_{1}^{i n} / v_{1}^{i n}$, and $k_{2}^{i n}=q_{2}^{i n} / v_{2}^{i n}$. In addition, this study assumes that the average traffic state over the highway segment are available, including the average density and flow on lane 1 and lane 2, which are defined as $k_{1}$ and $k_{2}, v_{1}$ and $v_{2}$ respectively.

To facilitate the mathematical analyses, this research introduces the following variables. We set the start point of the green zone as the origin of this zone (i.e., $x=0$ ) and introduces variables $q_{i}^{g}(x)$, $k_{i}^{g}(x)$ and $v_{i}^{g}(x)$ to denote the traffic flow, traffic density and traffic speed at a location $x$ on Lane $i=\{1,2\}$. Accordingly, we define $v_{i}^{g}$ to represent the average traffic speed of lane $i$ in the green zone. Similarly, we set the start point of the yellow zone as the origin of this zone (i.e. $z=0$ ) and introduce variables $q_{i}^{y}(z), k_{i}^{y}(z)$ and $v_{i}^{y}(z)$ to denote the traffic flow, traffic density and traffic speed at a location $\mathrm{z}$ on lane $i$ in the yellow zone. Accordingly, we define $k_{i}^{y}$ and $v_{i}^{y}$ to represent the average traffic density and speed of lane $i$ in the yellow zone respectively. Figure 1 also illustrates the necessary parameters and variables used for the proposed mathematical analyses.

To develop mathematical analyses and modeling, this study highlights the following assumptions, which will be used throughout the paper.

(I) There are $\alpha$ percentage vehicles on Lane 2 (also referred to as the subject lane) needs to perform MLC to Lane 1 (also referred to as the target lane) so that they can get off the highway at the off-ramp. A vehicle starting its mandatory lane change intention at the beginning of the green zone will complete 
it in the green zone. Otherwise, the vehicle will perform a lane change in the yellow zone.

(II) The discretionary lane change and its effect are not considered. This assumption also means that the lane change maneuvers from Lane 1 to Lane 2 in Figure 1 are not considered in the mathematical analysis and modeling.

(III) A lane change in the green zone will be completed smoothly without reducing driving speed (referred as S-MLC hereafter). A lane change in the yellow zone will be completed with speed deceleration (referred as D-MLC hereafter).

(IV) A vehicle will follow the traffic flow on the target lane immediately after successful perform an S-MLC or D-MLC in green or yellow zones (Daganzo, 2002a, b) ${ }^{4}$.

(V) The time delay resulting from the lane change is measured by the difference of the travel time that a vehicle goes through the road segments with or without MLC maneuvers in the traffic flow happens.

Regarding the assumption (II), the lane change in the opposite direction is ignored for the reasons below. First of all, the lane change maneuvers in the opposite direction usually happen to gain speed benefit. Thus, they are usually discretionary lane changes (DLCs) without speed deceleration. They won't cause significant traffic delay. Instead, they may potentially balance traffic flow on two lanes and benefit traffic delay reduction nearby off-ramp. However, DLC maneuvers usually happen randomly in both location and volume, which lead to significant difficulty to catch the effect in mathematical approach. This study also notices that ignoring the merit of DLC maneuvers may push the optimal advance warning a little bit further from the off-ramp, which is good from the safety point of view. On the other hand, existing research (Laval and Leclercq, 2008; Zheng, 2014) showed that the time delay resulting from DLC maneuvers is mainly caused by the relaxation phenomenon. Our research later will show that the relaxation phenomenon won't be a significant factor to affect the optimal location of the advance warning in the proposed research (see the discussion in Section 3.6 for the details). Furthermore, our numerical results also show that ignoring the DCLs in the opposite direction won't cause significant error for the proposed study.

Regarding the assumption (III), it is possible that the traffic condition changes gradually and may not be uniform in each zone in reality. Namely, S-MLC/D-MLC may happen in the yellow/green zone (our experiments actually observed it). The proposed research tries to approximate the reality while considering the methodical tractability. Thus, we only count the majority of MLC maneuvers around off-ramp. Namely, this study considers that most of MLC maneuvers in the green zone are smooth without speed deceleration and most MLC maneuvers in the yellow zone are rush with certain speed deceleration. These consideration is verified by our experiments in section 4.2.2. Of course, this simplification might cause some errors. Our experiment results indicate that the errors are within the acceptable range.

Relaying on the available data and assumptions, the research below presents our approach to identify the optimal lane change zones through mathematical models.

\section{Methodology}

This study first briefly discusses the effect of the D-MLC and S-MLC maneuvers on traffic flow before we present our methodology in details. According to the definitions of the green and yellow zones, a D-MLC maneuver in yellow zone will apparently slow down the downstream traffic and cause traffic delay on the subject lane. It is also recognized that a volume of S-MLC maneuvers in the green zone will cause time delay since they will gradually increase the traffic density on the target lane, even though a single S-MLC maneuver won't significantly impact traffic immediately. Thus, a vehicle running on the highway segment will be affected by both S-MLC maneuvers in the green zone and DMLC maneuvers in yellow zone. If the advance warning is located very close to the off-ramp, it will lead to many D-MLC maneuvers and cause traffic delay; on the other hand, if the advance warning is located too far away from the off-ramp so that S-MLC maneuvers are promoted, it might cause traffic congestion on the target lane and lead to high traffic delay, too. The simulation results in Figure 8

\footnotetext{
${ }^{4}$ Note that the bounded acceleration (the lane changer needs some time to adjust speed to follow the traffic flow which will affect the following vehicles) discussed in (Laval and Daganzo, 2006) is not considered to reduce the complexity of the time delay computation. Our experiment results indicate this assumption works well in this study.
} 
confirm this thought.

Therefore, it is critical to find an optimal location to give the advance warning, which will sustain enough opportunities for vehicles to perform safe and smooth S-MLC maneuvers, at the meantime, it minimizes the time delay resulting from both S-MLC and D-MLC maneuvers. Since the advance warning is given at the beginning of the green zone in this study. This is equal to find the optimal green zone and yellow zone before the off-ramp, considering the impacts of lane changes on traffic flow. The mathematical model denoted as the OPT-I presents our thoughts to identify the optimal green zone and yellow zone on the highway segment shown in Figure 1.

$$
\begin{array}{ll}
\text { OPT-I } & \min \quad \mathrm{Z}=T_{g}\left(\mathrm{~L}_{g}\right)+T_{y}\left(\mathrm{~L}_{y}\right) \\
\text { s.t. } & 0 \leq \mathrm{L}_{g}+\mathrm{L}_{y} \leq \mathrm{L}_{\text {Max }} \\
& \mathrm{L}_{g}, \mathrm{~L}_{y} \geq 0 \\
& 0 \leq \mathrm{F}_{g}\left(\mathrm{~L}_{g}\right) \leq 1 \\
& 0 \leq \mathrm{F}_{y}\left(\mathrm{~L}_{y}\right) \leq 1 \\
& \mathrm{~F}_{g}\left(\mathrm{~L}_{g}\right)+\left(1-\mathrm{F}_{g}\left(\mathrm{~L}_{g}\right)\right) \mathrm{F}_{y}\left(\mathrm{~L}_{y}\right) \geq \sigma
\end{array}
$$

Where, $L_{g}$ and $L_{y}$ are the decision variables which represent the location of the green zone and yellow zone respectively; $\left(L_{g}+L_{y}\right)$ determines the advance distance to give the advance warning for MLC maneuvers. $\mathrm{L}_{\operatorname{Max}}$ is the maximum system distance, which can be the length of the road. $\mathrm{F}_{g}$ and $\mathrm{F}_{y}$ are the function of $L_{g}$ and $L_{y}$, representing the probabilities that a vehicle may complete a S-MLC or D-MLC in the green zone or yellow zone; $\sigma$ is a pre-determined threshold, representing the acceptable ratio of the successful lane change opportunity as a vehicle runs through highway segment; Both $T_{g}$ and $T_{y}$ are the function of the variables $L_{g}$ and $L_{y}$; they represent the time delays resulting from SMLC or D-MLC maneuvers in the green and yellow zones respectively; $T_{g}$ and $T_{y}$ together demonstrate the impact of the MLC maneuvers occurring on the highway segment. According to assumption $(\mathrm{V}), T_{g}$ and $T_{y}$ are captured through comparing the travel times to go through the highway segment with and without the impact of the MLC (S-MLC and D-MLC) maneuvers. The objective of the OPT-I is to minimize this time delay resulting from these lane change maneuvers. The constraints ensure enough successfully lane change opportunities for individual vehicles. Clearly, the time delay $\left(T_{g}\right.$ and $\left.T_{y}\right)$ and the lane change opportunities $\left(F_{g}\right.$ and $\left.F_{y}\right)$ are related to the location of the green zone and yellow zone (i.e. $L_{g}$ and $L_{y}$ ) as well as traffic flow condition on the highway segment. In addition, as the advance warning is set at different locations, the corresponding green and yellow zones will vary, so do lane change opportunities and time delay. The research below presents our approaches to specify the elements in the OPT-I and the corresponding solution method to find the optimal solution.

\subsection{The opportunity of lane change}

This study first investigates the formulation to calculate the opportunities that a vehicle may successfully complete an S-MLC or D-MLC in the green zone or yellow zone. We first develop the approach for the green zone, and then show its applicability to the yellow zone. It is noticed that many advanced lane change decision models in literature (Ahmed, 1999; Toledo et. al., 2003; Toledo et. al., 2007) are applicable to estimate this probability with different accuracy. For simplicity, this study estimates this probability according to lane change decision model given in (S. Gurupackiam etc. 2010), by which a vehicle will perform a successful safe lane change if the spacing headway on target lane $(h)$ is larger than the accepted gap $(g)$. Considering $g$ and $h$ as two independent random variables with the probability density function (pdf) $\mathrm{v}(\mathrm{h})$ and $\lambda(\mathrm{g})$ respectively, we can find the probability of a successful lane change for a given gap at target lane by Equation (1).

$$
\mathrm{p}=\mathrm{P}(\mathrm{h} \geq g)=\iint_{D} f(h, g) d h d g=\int_{0}^{+\infty} v(h) \int_{0}^{h} \lambda(g) d g d h
$$

Built upon Equation (1), we develop the formulation to capture the opportunity to complete a successful S-MLC in the green zone. It is observed that every time when a vehicle passes a gap in the target lane (Lane 1), it will have a chance. If a successful lane change happens at the $n^{\text {th }}$ gap, then it fails in the first $n-1$ times. Assuming that the traffic flow keeps stable during the course that the 
vehicle searches for a gap and conducts an S-MLC, it is recognized that each attempt of the vehicle will have an identical and independent probability to success. Then, the number of the attempts before a successful S-MLC follows a geometric distribution with the cumulative distribution function given below.

$$
\mathrm{F}(n)=1-(1-\mathrm{p})^{n}
$$

Next, we explore the formulation to estimate the value of $n$. Assuming during that time period that a vehicle searches safe lane change gap, both vehicle $u$ on the subject lane, Lane 2 and vehicle $v$ on the target lane, Lane 1 keep constant speed $v_{u}$ and $v_{v}$, and the average traffic density on Lane 1 is $k_{1}^{g}$, it is observed that the relative movement between vehicle $u$ and vehicle $v$ is measured by $\left|x_{u}-x_{v}\right|$, and vehicle $u$ will only meet the gaps in this range. As a result, we estimate the number of gaps $\left(n_{u}\right)$ that vehicle $u$ will meet by the product of the relative movement and the average traffic density on Lane 1 in the green zone. Equation (3) below presents this idea.

$$
n_{u}=\left|x_{u}-x_{v}\right| k_{1}^{g}=\frac{x_{u}}{v_{u}}\left|v_{u}-v_{v}\right| k_{1}^{g}+1
$$

The above equation estimates the lane change opportunity for a vehicle by capturing its microscopic mobility. This study, then, proposes the formulation in Equation (4), which follows the same logic in Equation (3) but factors macroscopic traffic flow features on Lane 1 and Lane 2.

$$
\mathrm{n}=\frac{x}{v_{2}^{g}}\left|v_{2}^{g}-v_{1}^{g}\right| k_{1}^{g}+1
$$

Where, $x$ represents the distance that a vehicle moves in the green zone before it finds a safe lane change gap; $v_{2}^{g}$ and $v_{1}^{g}$ represent the average traffic speed of Lane 2 and Lane 1 in the green zone.

Plugging Equation (4) into (2), this study estimates the probability of finding a safe lane change spot in the range of $(0, x)$ in the green zone by Equation (5).

$$
\mathrm{F}_{g}(x)=\mathrm{F}_{g}(0 \leq \mathrm{X} \leq x)=1-(1-\mathrm{p})^{\frac{x}{v_{2}^{g}}\left|v_{2}^{g}-v_{1}^{g}\right| k_{1}^{g},+1}
$$

Accordingly, $\mathrm{F}_{g}\left(L_{g}\right)$, in which $x_{g}$ equals to its maximum value $L_{g}$, represents the probability of finding a safe lane change spot if a vehicle goes through the green zone. This method can be adopted to the yellow zone by using corresponding elements in the yellow zone. Equation (6) presents the formulation.

$$
\mathrm{F}_{y}(0 \leq \mathrm{Z} \leq z)=1-(1-\mathrm{p})^{\frac{z}{v_{2}^{y}}\left|v_{2}^{y^{\prime}}-v_{1}^{y}\right| k_{1}^{y}}
$$

Without knowing the location of the green zone, it is hard to estimate $v_{2}^{g}, v_{1}^{g}$ and $k_{1}^{g}$ as well as $v_{2}^{y \prime}$, $v_{1}^{y}$ and $k_{1}^{y}$ accurately in Equations (5) and (6). This study thus will approximate them by the average density and speed over the highway segment. Namely, we make $v_{1}^{g}=v_{1}, v_{2}^{g}=v_{2}, k_{1}^{g}=k_{1}$ as well as $v_{1}^{y}=v_{1}, v_{2}^{y}=v_{2} k_{1}^{y}=k_{1}$ in Equations (5) and (6) to obtain Equations (7) and (8). We denote that the pdf of $\mathrm{F}_{g}(\mathrm{x})$ as $p_{g}(x)$, and the pdf of $\mathrm{F}_{y}(\mathrm{Z})$ as $p_{y}(z)$. This approximation is a compromise. Our experiment results indicate that it won't lead significant error.

$$
\begin{gathered}
\mathrm{F}_{g}(0 \leq \mathrm{X} \leq x)=1-(1-\mathrm{p})^{\frac{x}{v_{2}}\left|v_{2}-v_{1}\right| k_{1},+1} \\
\mathrm{~F}_{y}(0 \leq \mathrm{Z} \leq z)=1-(1-\mathrm{p})^{\frac{z}{v_{2}}\left|v_{2}^{y^{\prime}}-v_{1}\right| k_{1}}
\end{gathered}
$$

According to Equations (7) and (8), this study briefly discusses the lane change maneuvers and the need of the advance warning at different traffic conditions. When the traffic flow is very sparse, $\mathrm{p} \rightarrow 1$ and $\mathrm{F}_{a}(0 \leq \mathrm{X} \leq x) \rightarrow 1$ at any location $x$. It means that vehicles can smoothly complete lane change at any location of the road segment. Then, a later advance warning will work ok. On the other hand, if the traffic flow is very dense, the value of $\mathrm{p}$ becomes very small. The traffic flow on both Lane 1 and Lane 2 will run in a very slow speed, thus $v_{2} \approx v_{1}, \mathrm{~F}_{g}(0 \leq \mathrm{X} \leq x) \rightarrow 0$. It indicates that smooth lane change becomes very difficult due to traffic congestion. An earlier advance warning is needed so that vehicles can start the lane change intention earlier to avoid rush maneuvers. At the extreme situation, vehicles will decelerate and try to find more chance to do a successful lane change even the earlier advance warning is given. Then only yellow zone exists at the downstream of the advance warning exists. Except the extreme traffic condition, the green zone and yellow zone may coexist. Our numerical experiments presented in Section 4 will provide the discussion in details. 
In addition, even though this study applied the average/constant successful probability for a single lane change attempt in the whole road segment. However, Equations (7) and (8) indicate that green zone and yellow zone will grant different probabilities to find acceptable gaps according to their traffic condition and lengths. Accordingly, vehicles will have different probabilities to successfully complete mandatory lane changes in green zone and yellow zones. This makes our model to approach traffic reality as much as possible.

\subsection{Traffic evolution caused by S-MLC in the green zone}

This section studies the traffic flow change and the corresponding traffic delay due to the S-MLC maneuvers in the green zone. According to assumption (III), each S-MLC maneuver won't interrupt/slow down traffic flow on either the subject lane, Lane 1 or the target lane, Lane 2 significantly, but a volume of S-MLC maneuvers may gradually increase traffic flow on Lane 1 and finally cause apparent delay. Along with this recognition, this study will first investigate the gradual traffic state change on both Lane 1 and Lane 2 and then propose the approaches to capture the delay experienced by vehicles in the green zone. To do this, we consider that traffic flow entering the green zone is consistent to the traffic flow entering the road segment, i.e., $q_{i}^{g}(0)=q_{i}^{i n}, k_{i}^{g}(0)=k_{i}^{i n}, v_{i}^{g}(0)=$ $v_{i}^{i n}, i=1,2$. In other words, even though the start point of the green zone is unknown yet, its original traffic states including density, speed, and flow are detectable.

To analyze the gradual change of traffic flow on Lane 1 and Lane 2, this study slices the green zone into a number of small cells and each of such cells with a length $\Delta x$, as shown in Figure 2 . The start point of the green zone is considered as the original point (i.e. $\mathrm{x}=0$ ). For a cell at location $x$ on Lane $i$, its inflow is denoted as $q_{i}^{g}(x-\Delta x)$, and the outflow is denoted as $q_{i}^{g}(x)$. The lane changes from a cell at $x$ on Lane 2 to another cell on Lane 1 leads to traffic flow change, which is calculated by $\alpha p_{g}(x) \Delta x q_{2}^{i n}$. Note that $\left(p_{g}(x) \Delta x\right)$ is the probability of a successful lane change which starts the attempt at the initial point of the green zone and successes in the cell $x$, given $p_{g}(x)$ is calculated by Equation (5); and $q_{2}^{\text {in }}$ represents the flow at the initial point of the green zone in Lane 2.

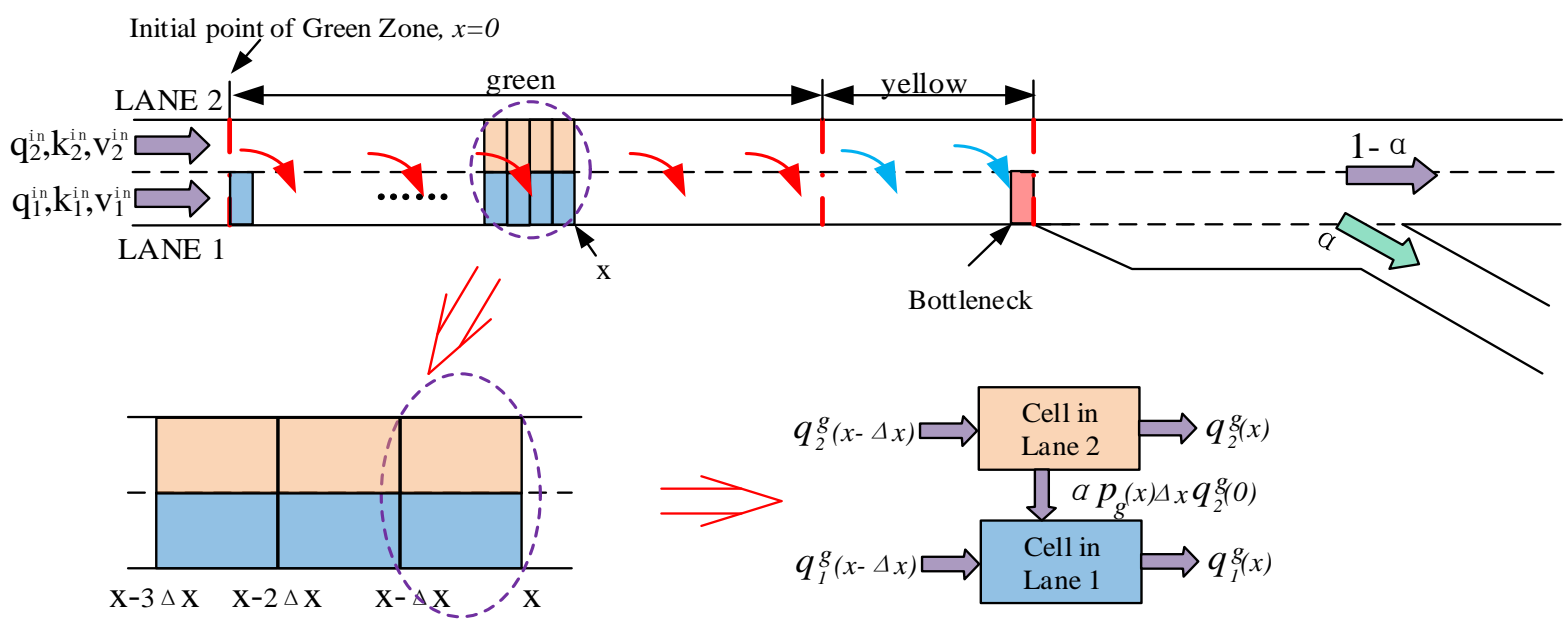

Figure 2. Gradual traffic state evolution analysis for Lane 1

According to flow conservation, we obtain Equations (9) and (10) below.

$$
\begin{aligned}
& q_{1}^{g}(x)=q_{1}^{g}(x-\Delta x)+\alpha\left(p_{g}(x) \Delta x\right) q_{2}^{i n} \\
& q_{2}^{g}(x)=q_{2}^{g}(x-\Delta x)-\alpha\left(p_{g}(x) \Delta x\right) q_{2}^{\text {in }}
\end{aligned}
$$

Next, we develop the formulations to estimate the traffic flow in next cell by use the results in Equations (9) and (10).

$$
\begin{aligned}
& q_{1}^{g}(x+\Delta x)=q_{1}^{g}(x)+\alpha p_{g}(x+\Delta x) q_{2}^{i n}=q_{1}^{g}(x-\Delta x)+\alpha\left[p_{g}(x+\Delta x)+p_{g}(x)\right] \Delta x q_{2}^{i n} \\
& q_{2}^{g}(x+\Delta x)=q_{2}^{g}(x)-\alpha p_{g}(x+\Delta x) q_{2}^{i n}=q_{2}^{g}(x-\Delta x)-\alpha\left[p_{g}(x+\Delta x)+p_{g}(x)\right] \Delta x q_{2}^{i n}
\end{aligned}
$$

After more iterations, it is observed that:

$$
\begin{aligned}
& q_{1}^{g}(x)=q_{1}^{\text {in }}+\alpha\left[p_{g}(x-\Delta x)+p_{g}(x-2 \Delta x)+\cdots+p_{g}(\Delta x)\right] \Delta x q_{2}^{\text {in }} \\
& q_{2}^{g}(x)=q_{2}^{\text {in }}-\alpha\left[p_{g}(x-\Delta x)+p_{g}(x-2 \Delta x)+\cdots+p_{g}(\Delta x)\right] \Delta x q_{2}^{\text {in }}
\end{aligned}
$$


As $\Delta x$ is very small, the sum of $p_{g}(x-n \Delta x) \Delta x$ is the cumulative probability that an S-MLC successes in the range of $[0, \mathrm{x}]$. As $\Delta x \rightarrow 0$, we calculate the cumulative probability in Equation (15).

$$
\mathrm{F}_{g}(x)=\int_{0}^{x} p_{g}(x) d x
$$

Then, the generalized formulations to capture traffic flow at any location $x$ on Lane 1 and Lane 2 are presented in Equations (16) and (17) below.

$$
\begin{aligned}
& q_{1}^{g}(x)=q_{1}^{i n}+\alpha \mathrm{F}_{g}(x) q_{2}^{i n} \\
& q_{2}^{g}(x)=q_{2}^{\text {in }}-\alpha \mathrm{F}_{g}(x) q_{2}^{\text {in }}
\end{aligned}
$$

Equation (16) indicates that the S-MLC maneuvers will increase the traffic flow on Lane 1 and drive it to a peak value around the area of the off-ramp. Thus it is possible to make this area become a bottleneck. If the downstream traffic is not very smooth, it is apt to generate traffic congestion there. On the other hand, the S-MLC maneuvers may lighten the traffic flow on Lane 2 near to the off-ramp. These observations are consistent to traffic reality well documented in literature. Combining equation (5), (16), (17), and Greenshield model, this study provides the formulations (18) and (19) to capture the traffic speed and density at any location $x$ on lane $i$ in the green zone under free flow $\left(k_{i}^{i n}<k_{m}\right)$ and congestion flow $\left(k_{i}^{\text {in }} \geq k_{m}\right)$ respectively.

$$
\begin{array}{ll}
v_{i}^{g}(\mathrm{x})= \begin{cases}\frac{v_{f}}{2}\left[1+\sqrt{1-4 \frac{q_{i}^{g}(x)}{k_{j} v_{f}}}\right] & k_{i}^{i n}<k_{m} \\
\frac{v_{f}}{2}\left[1-\sqrt{1-4 \frac{q_{i}^{g}(x)}{k_{j} v_{f}}}\right] & k_{i}^{i n} \geq k_{m}\end{cases} \\
k_{i}^{g}(\mathrm{x})= \begin{cases}\frac{1}{2}\left[k_{j}-k_{j} \sqrt{1-4 \frac{q_{i}^{g}(x)}{k_{j} v_{f}}}\right] & k_{i}^{i n}<k_{m} \\
\frac{1}{2}\left[k_{j}+k_{j} \sqrt{1-4 \frac{q_{i}^{g}(x)}{k_{j} v_{f}}}\right] & k_{i}^{i n} \geq k_{m}\end{cases}
\end{array}
$$

Where, $v_{f}, k_{j}, k_{m}=k_{j} / 2$ represent the free flow speed, jam density, and optimal density respectively.

Moreover, it is noticed that there are $k_{i}^{g}(\mathrm{x}) \Delta x$ amount of vehicles in the cell of Lane $i$ at location $x$ in the green zone. Each vehicle follows a speed $v_{i}^{g}(\mathrm{x})$ in this cell. Accordingly, there are $\mathrm{N}=\int_{0}^{L g} k_{i}^{g}(\mathrm{x}) d x$ vehicles on Lane $i$ in the green zone. Then, we can find the average speed in each lane by Equation (20).

$$
v_{i}^{g}=\int_{0}^{L_{g}} v_{i}^{g}(\mathrm{x}) k_{i}^{g}(x) d x / \int_{0}^{L_{g}} k_{i}^{g}(\mathrm{x}) d x, i=1,2
$$

Equations (16) - (20) enable us to analyze the gradual traffic state change on both the subject lane and target lane along the green zone, considering the probabilistic S-MLC maneuvers. The same method can also be used to analyze the gradual traffic state change on target lane in yellow zone, which results from the effect of the D-MLC maneuvers, according to our assumption (IV) that vehicles will flow the target lane speed immediately after a successful lane change is conducted.

\subsection{Time delay caused by S-MLC}

It is observed that the S-MLC maneuvers in the green zone will affect the travel time of the vehicles, which follow three different trajectories to go through the road segment. Below develops the formulations to capture their respective delay resulting from the S-MLC maneuvers in the green zone. According to the definition of the time delay given in assumption $(\mathrm{V})$, we capture the time delay by the travel time difference for a vehicle to go through the green zone by speed $v_{i}^{g}(\mathrm{x})$, which counts the effect of the S-MLC maneuvers, and $v_{i}^{i n}$, which is the speed that vehicles entering the highway segment 
without counting the effect of the S-MLC maneuvers.

Type I flow, denoted as $q_{1 \rightarrow 1}$, will stay on Lane 1 and go through the green zone, i.e., $q_{1 \rightarrow 1}=q_{1}^{i n}$. Thus, it will experience a delay $\left(t_{1 \rightarrow 1}^{g}\right)$ due to the gradual traffic variation (i.e., the traffic gradually becomes denser) resulting from the S-MLC maneuvers. Equation (21) calculates the delay, in which $v_{1}^{g}(\mathrm{x})$ is formulated by Equation (18), in which $i=1$.

$$
t_{1 \rightarrow 1}^{g}=\int_{0}^{L_{g}}\left(\frac{d x}{v_{1}^{g}(\mathrm{x})}-\frac{d x}{v_{1}^{g}(0)}\right) \stackrel{v_{1}^{g}(0)=v_{1}^{i n}}{\Longleftrightarrow} \int_{0}^{L_{g}}\left(\frac{d x}{v_{1}^{g}(\mathrm{x})}-\frac{d x}{v_{1}^{i n}}\right)
$$

Type II flow will stay on Lane 2 and go through the green zone. It contains two groups of vehicles: (i) they will go through the green zone and yellow zone without lane change maneuvers, i.e., $q_{2 \rightarrow 2}=$ $(1-\alpha) q_{2}^{\text {in }}+\left(1-F_{g}\left(L_{g}\right)\right)\left(1-F_{y}\left(L_{y}\right)\right) q_{2}^{\text {in }}$; (ii) they cannot complete the S-MLC maneuvers in the green zone but do successful D-MLC maneuvers in the yellow zone, $q_{2 \rightarrow 1}^{y}=F_{y}\left(L_{y}\right)(1-$ $\left.F_{g}\left(L_{g}\right)\right) \alpha q_{2}^{i n}$. Thus, Type II flow will experience a delay $\left(t_{2 \rightarrow 2}^{g}\right)$ due to the gradual traffic evolution on Lane 2 (i.e., the traffic gradually becomes sparser) resulting from the S-MLC maneuvers. Equations (22) below enables the calculation of the delay, in which traffic speed $v_{2}^{g}(x)$ is captured by the formulation given in Equation (18), in which $i=2$.

$$
t_{2 \rightarrow 2}^{g}=\int_{0}^{L_{g}}\left(\frac{d x}{v_{2}^{g}(x)}-\frac{d x}{v_{2}^{g}(0)}\right) \stackrel{v_{2}^{g}(0)=v_{2}^{i n}}{\Longleftrightarrow} \int_{0}^{L_{g}}\left(\frac{d x}{v_{2}^{g}(x)}-\frac{d x}{v_{2}^{\text {in }}}\right)
$$

Type III flow, denoted as $q_{2 \rightarrow 1}^{g}$, includes the vehicles which experience driving on Lane 2 before a successful S-MLC and then on Lane 1 after this maneuver, i.e., $q_{2 \rightarrow 1}^{g}=\alpha \mathrm{F}_{g}\left(L_{g}\right) q_{2}^{i n}$. Assuming a vehicle successfully performs lane change at point $r$, Equation (23) captures the delay.

$$
\begin{aligned}
& T_{2 \rightarrow 1}^{g}(r)=\int_{0}^{r}\left(\frac{d x}{v_{2}^{g}(x)}-\frac{d x}{v_{2}^{g}(0)}\right)+\int_{r}^{L_{g}}\left(\frac{d x}{v_{1}^{g}(x)}-\frac{d x}{v_{2}^{g}(0)}\right) \\
& =\int_{0}^{r} \frac{d x}{v_{2}^{g}(x)}+\int_{r}^{L_{g}} \frac{d x}{v_{1}^{g}(x)}-\int_{0}^{L_{g}} \frac{d x}{v_{2}^{g}(0)} \\
& =\int_{0}^{r} \frac{d x}{v_{2}^{g}(x)}+\int_{r}^{L_{g}} \frac{d x}{v_{1}^{g}(x)}-\frac{L_{g}}{v_{2}^{g}(0)} \\
& \stackrel{v_{2}^{g}(0)=v_{2}^{i n}}{\rightleftharpoons} \int_{0}^{r} \frac{d x}{v_{2}^{g}(x)}+\int_{r}^{L_{g}} \frac{d x}{v_{1}^{g}(x)}-\frac{L_{g}}{v_{2}^{i n}}
\end{aligned}
$$

Equation (23) indicates that the delay depends on the location $\left(r \in\left[0, L_{g}\right]\right)$ where a successful S-MLC happens. Equation (5) demonstrates that a successful lane change that happens in a range $(0, r)$ in the green zone follows a distribution $F_{g}(r)$. (Its pdf is denoted as $p_{g}(r)$ ). Then, $T_{2 \rightarrow 1}^{g}(r)$ is a function of random variable $r$. This study computes the expected delay $\left(t_{2 \rightarrow 1}^{g}\right)$ for a Type III vehicle by Equation (24) below, in which $v_{1}^{g}(\mathrm{x})$ and $v_{2}^{g}(\mathrm{x})$ will be calculated by Equation (18), where $i=1,2$ respectively.

$$
\begin{aligned}
& t_{2 \rightarrow 1}^{g}=E\left(T_{2 \rightarrow 1}^{g}\right)=\int_{0}^{L_{g}} T_{2 \rightarrow 1}^{g} p_{g}(r) d r=\int_{0}^{L_{g}} T_{2 \rightarrow 1}^{g} d F_{g}(r) \\
& =\left.T_{2 \rightarrow 1}^{g} F_{g}(r)\right|_{0} ^{L_{g}}-\int_{0}^{L_{g}} F_{g}(r) d T_{2 \rightarrow 1}^{g} \\
& =\left.T_{2 \rightarrow 1}^{g} F_{g}(r)\right|_{0} ^{L_{g}}-\int_{0}^{L_{g}} F_{g}(r)\left(\frac{1}{v_{2}^{g}(\mathrm{r})}-\frac{1}{v_{1}^{g}(\mathrm{r})}\right) d r
\end{aligned}
$$

It is noticed that the total flow entering the green zone is $\left(q_{1}^{i n}+q_{2}^{i n}\right)$. The three types of vehicles form three types of flows. Then, the average time delay $\left(T_{g}\right)$ experienced by a vehicle in the green zone is given below.

$$
E\left(T_{g}\right)=t_{1 \rightarrow 1}^{g}\left(\frac{q_{1 \rightarrow 1}}{q_{1}^{i n}+q_{2}^{i n}}\right)+t_{2 \rightarrow 2}^{g}\left(\frac{q_{2 \rightarrow 2}+q_{2 \rightarrow 1}^{y}}{q_{1}^{i n}+q_{2}^{\text {in }}}\right)+t_{2 \rightarrow 1}^{g}\left(\frac{q_{2 \rightarrow 1}^{g}}{q_{1}^{\text {in }}+q_{2}^{\text {in }}}\right)
$$

Appendix A presents the closed-form formulations for $t_{1 \rightarrow 1}^{g}, t_{2 \rightarrow 2}^{g}$, and $t_{2 \rightarrow 1}^{g}$. Specifically, $t_{1 \rightarrow 1}^{g}$ is 
calculated in Equations (54) and (55) as Lane 1 is under free flow and congested flow conditions, respectively. $t_{2 \rightarrow 2}^{g}$ is calculated in Equations (56) and (57) as Lane 2 is under free flow and congested flow conditions, respectively. $t_{2 \rightarrow 1}^{g}$ is calculated in Equation (58) under the condition that both Lane 1 and Lane 2 are under free flow. $t_{2 \rightarrow 1}^{g}$ is calculated in Equation (59) under the condition that both Lane 1 and Lane 2 are under congestion flow. $t_{2 \rightarrow 1}^{g}$ is calculated in Equation (60) under the condition that Lane 1 is under free flow and Lane 2 is under congestion flow. $t_{2 \rightarrow 1}^{g}$ is calculated in Equation (61) under the condition that Lane 1 is under congestion flow and Lane 2 is under free flow.

\subsection{Traffic fluctuation caused by D-MLC in yellow zone}

According to assumptions (I) and (III), a vehicle which cannot complete an S-MLC in the green zone will conduct a lane change in yellow zone with speed deceleration (i.e. a D-MLC). The deceleration will generate shock waves and cause traffic delay on the subject lane (Lane 2). On the other hand, according to assumption (IV), a vehicle will follow the speed on the target lane (Lane 1) immediately after it completes a D-MLC. Then, the traffic on Lane 1 in yellow zone will also experience a gradual traffic state evolution. With these views, the presented research below studies traffic flow on Lane 1 and Lane 2 in the yellow zone separately, and then develops the formulations to capture the time delay resulting from D-MLC maneuvers. Before presents our methodology, this study recalls the traffic flow conversation law and recognizes that $q_{i}^{y}(0)=q_{i}^{g}\left(L_{g}\right), k_{i}^{y}(0)=k_{i}^{g}\left(L_{g}\right), v_{i}^{y}(0)=v_{i}^{g}\left(L_{g}\right), i=$ 1,2 , for yellow zone. Namely, the entering flow to the yellow zone is equal to the exit flow at the end of the green zone. $q_{i}^{g}\left(L_{g}\right), k_{i}^{g}\left(L_{g}\right)$, and $v_{i}^{g}\left(L_{g}\right)$ can be calculated by Equations (16) to (19).

\subsubsection{Traffic flow evolution and time delay on Lane 1}

The traffic evolution on the target lane, Lane 1, in yellow zone will be analyzed through the same approaches used to study traffic on Lane 1 in the green zone in Section 3.2. Thus, given $\alpha$ percent of traffic flow $\left(q_{2}^{\text {in }}\right)$ entering Lane 2 needs change to Lane 1, and each of them successes in the green zone with a probability $F_{g}\left(L_{g}\right)$, there are $\left(1-F_{g}\left(L_{g}\right)\right) \alpha q_{2}$ amount of flows which will have to perform the D-MLC maneuvers in the yellow zone. Consequently, the percentage (i.e. probability, $\beta$ ) of those vehicles on Lane 2 which will conduct a D-MLC in yellow zone can be calculated by Equation (26).

$$
\beta=\frac{\alpha\left[1-F_{g}\left(L_{g}\right)\right]}{1-\alpha F_{g}\left(L_{g}\right)}
$$

Following the method in Equation (16), which calculates the traffic flow evolution on Lane 1 due to S-MLC maneuvers in the green zone, Equation (27) below captures the traffic flow at any location $Z$ on Lane 1 in yellow zone.

$$
q_{1}^{y}(z)=q_{1}^{y}(0)+\beta \mathrm{F}_{y}\left(L_{y}\right) q_{2}^{y}(0)
$$

Similarly, Equations (18), (19) and (20) are adoptable to calculate $v_{1}^{y}(\mathrm{z}), k_{i}^{y}(\mathrm{z})$ and average speed $v_{1}^{y}$, using corresponding parameters in the yellow zone. Moreover, the average density on Lane 1 in yellow zone is in Equations (28) below.

$$
k_{1}^{y}=\frac{\int_{0}^{L_{y}} k_{i}^{y}(\mathrm{z}) d z}{L_{y}}
$$

Last, the time delay in the yellow zone experienced by a vehicle always staying on Lane 1 can be calculated by the idea presented in the Equation below.

$$
t_{1 \rightarrow 1}^{y}=\int_{0}^{L_{y}}\left(\frac{d z}{\mathrm{v}_{1}^{y}(\mathrm{z})}-\frac{d z}{\mathrm{v}_{1}^{\text {in }}}\right)
$$

The above methods are not proper to analyze the traffic states and delay on Lane 2 in the yellow zone due to the shock wave caused by the D-MLC maneuvers. This study presents different methods in next several sections.

\subsubsection{D-MLC groups on Lane 2}

It is observed that a vehicle performing a D-MLC will affect its following vehicles, but not its leading vehicle. This effect will stop at another vehicle behind, which is also conducting a D-MLC. Thus, this study separates the vehicle platoon on Lane 2 in the yellow zone into multiple D-MLC groups. 


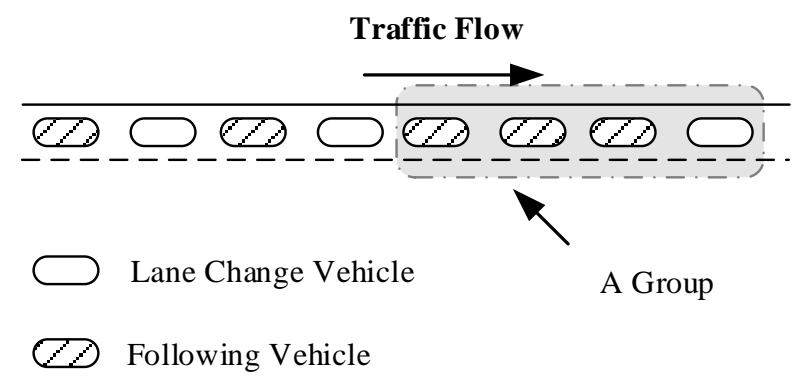

Figure 3. D-MLC Group in the yellow zone

Each of such groups includes $m$ vehicles consisted of a leading vehicle conducting a D-MLC and $m-1$ following vehicles. Figure 3 shows an example. Each vehicle in the yellow zone is either a leading vehicle performing a D-MLC or a following vehicle in a D-MLC group. Considering each vehicle in the platoon might be a leading vehicle in a D-MLC group independently with the probability equals to $\beta$, we study the random variable $m$, which represents the first vehicle conducting a D-MLC in the platoon. It is recognized that $m$ follows a geomantic distribution. Accordingly, there are $m$ vehicles in a D-MLC group can be calculated by Equation (30).

$$
f(m)=(1-\beta)^{m-1} \beta
$$

\subsubsection{Shock waves on Lane 2}

Next, this study investigates the shock wave generated by each D-MLC. Given a vehicle conducting a D-MLC in the yellow zone will decelerate its speed from $v_{2}^{y}$ to $v_{2}^{y^{\prime}}$, the shock wave speed can be calculated by Equation (31) below.

$$
v_{w}=\frac{q_{2}^{y}-q_{2}^{y} \prime}{k_{2}^{y}-k_{2}^{y},}=v_{2}^{y}+v_{2}^{y^{\prime}}-v_{f},
$$

where Greenshield model, $k_{2}^{y^{\prime}}=k_{j}\left(1-\frac{v_{2}^{y \prime}}{v_{f}}\right)$, is used to estimate the traffic density, and the identity $(q=k v)$ is used to derive traffic flow according to density and speed. Apparently, $v_{w} \geq 0$ or $v_{w} \leq 0$ indicates a forward or backward shock wave corresponding different traffic condition.

As $v_{w}<0$, the shock wave propagates backward. According to the traffic speed $v_{1}^{y}$ on Lane 1 and the traffic speed $v_{2}^{y}$ on Lane 2 in the yellow zone, the deceleration of a vehicle aiming to increase the opportunities for a safe D-MLC (i.e. increasing the relative speed between the subject vehicle and the target lane so that the vehicle can meet more gaps) implies two scenarios of the speed relationships: (i) $v_{2}^{y^{\prime}}<v_{2}^{y}<v_{1}^{y}$, given $v_{2}^{y}<v_{1}^{y}$. (ii) $v_{2}^{y}-v_{1}^{y}<v_{1}^{y}-v_{2}^{y \prime}$ and $v_{2}^{y^{\prime}}<v_{1}^{y}$, given $v_{1}^{y}<v_{2}^{y}$. Since a backward shock wave (i.e. $v_{2}^{y}+v_{2}^{y^{\prime}}-v_{f}<0$ ) happens, both Scenarios (i) and (ii) imply $v_{2}^{y_{\prime}}<\frac{v_{f}}{2}$ and $v_{2}^{y}<v_{f}-v_{2}^{y_{\prime}}$. Hence, we conclude that a backward shock wave will be generated by a D-MLC, if the subject lane, Lane 2, in yellow zone is under congestion and a vehicle performing a DMLC runs in a speed less than optimal speed $\left(\frac{v_{f}}{2}\right)$.

As $v_{w}>0$, the shock wave broadcasts forward. Again, the deceleration of a vehicle aiming to increase the opportunities for a safe a D-MLC implies two scenarios of the speed relationships. (i) $v_{2}^{y^{\prime}}<v_{2}^{y}<v_{1}^{y}$, if $v_{2}^{y}<v_{1}^{y}$. (ii) $v_{2}^{y}-v_{1}^{y}<v_{1}^{y}-v_{2}^{y \prime}$ if $v_{2}^{y^{\prime}}<v_{1}^{y}<v_{2}^{y}$. Given a forward shock wave happens (i.e. $v_{2}^{y}+v_{2}^{y^{\prime}}-v_{f}>0$ ), Scenarios (i) and (ii) imply $v_{1}^{y}>v_{2}^{y}>\frac{v_{f}}{2}$ or $v_{2}^{y}>v_{1}^{y}>\frac{v_{f}}{2}$ respectively. They both indicate that a forward shock wave will be generated if both the subject lane, Lane 2 and the target lane, Lane 1 in yellow zone are under a better traffic condition such as traffic speed greater than optimal speed $\left(\frac{v_{f}}{2}\right)$.

To perform the shock analysis, we include two more assumptions: (a) a vehicle without the lane change need (it will be a following vehicle in a D-MLC group) will keep the speed as it enters the yellow zone, i.e., $v_{2}^{y}=v_{2}^{y}(0)=v_{2}^{g}\left(L_{g}\right)$ until it is affected by a shock wave; (b) a vehicle with the lane change need (it will be the leading vehicle in a D-MLC group) will slow down from $v_{2}^{y}$ to $v_{2}^{y \prime}$ at the start point of the yellow zone to search for a gap for lane change. In other words, the shock wave will start at the initial point of the yellow zone which also is the end point of the green zone. Both the 
forward shock wave and backward shock wave will affect the vehicles downstream of the vehicle performing lane change. But forward shock wave moves forward, thus, only affects the vehicles in the yellow zone. Backward shock wave will move backward and possibly affect vehicles on the whole road segment. The follows investigate the time delay corresponding to the forward and backward shock wave.

\subsubsection{Traffic delay resulting from the forward shock wave}

This section studies the effect of the forward shock wave on the traffic flow on Lane 2 in the yellow zone. Figure 4 illustrates the effect of the forward shock waves, where we consider the vehicles need to perform D-MLC maneuvers are randomly distributed in the traffic flow. A D-MLC maneuver starting at $(z=0, t=0)$ generates a shock wave (OB) with speed $v_{w}$, which slows down the following vehicles in a D-MLC group to speed $v_{2}^{y \prime}$. After the vehicle completes the D-MLC and left Lane 2 at point $\mathrm{A}$, the following vehicles will restore to their previous speed $v_{2}^{y}$, thus produce another shock wave AC parallel to the shock wave OB. The shock wave OB stops at point $\mathrm{B}$, where another vehicle already decelerates to speed $v_{2}^{y \prime}$ to conduct a D-MLC. For the same reason, the shock wave AC will stop at point $\mathrm{C}$, where it hits a vehicle which already restore its speed to the previous speed $v_{2}^{y}$. In reality, the following vehicle may restore to a speed which is faster than the previous speed $v_{2}^{y}$ since the traffic on Lane 2 may become sparse due to the lane-change maneuvers. However, it is very hard to determine the restored speed exactly, thus we use its previous speed $v_{2}^{y}$ as an approximation.

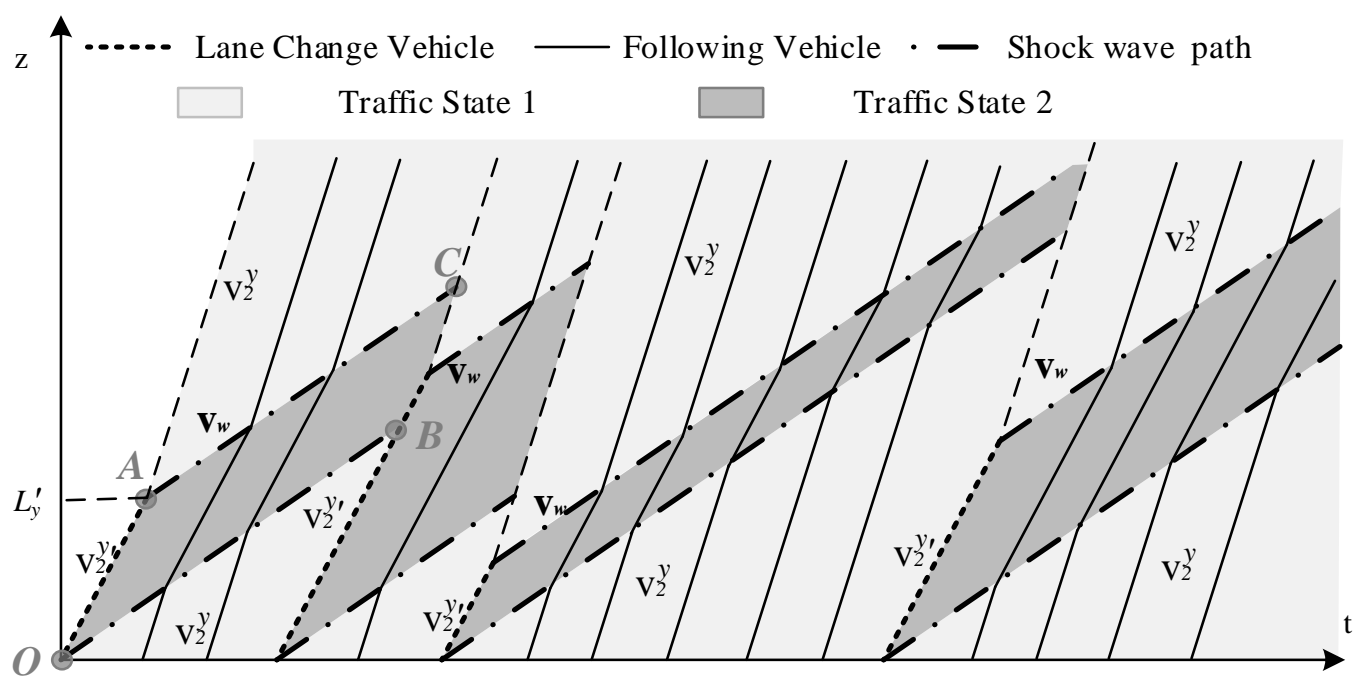

Figure 4. Illustration of forward shock wave

Based on the above analyses, this study develops the formulation to measure the time delay on Lane 2 resulting from a D-MLC. The examples in Figure 4 indicates that a leading vehicle in a D-MLC group will take the time of $\frac{L_{y}^{\prime}}{v_{2}^{y}}$ to go through a distance $L_{y}^{\prime}$, in which it can complete searching and conducting a safe D-MLC. All the affected following vehicles in a D-MLC group will slow down to the speed $v_{2}^{y \prime}$ sequentially, experience the same amount of time period to travel a distance $L_{y}^{\prime}$, and then restore to speed $v_{2}^{y}$. Hence, according to the time delay definition given in assumption $(\mathrm{V})$, the time delay of both the leading vehicle and the affected following vehicles in a D-MLC group is calculated by Equation (32) .

$$
t_{y f}=\frac{L_{y}^{\prime}}{v_{2}^{y_{\prime}}}-\frac{L_{y}^{\prime}}{v_{2}^{i n}}
$$

Next, we develop the formulation to calculate $L_{y}^{\prime}$. In the worst case, a leading vehicle needs to go through the whole range of the yellow zone, i.e., $L_{y}^{\prime}=L_{y}$. If we consider the vehicle keeps constant speed $\left(v_{2}^{y \prime}\right)$, and the traffic flow on target lane keeps stable (constant $v_{1}^{y}$ and uniform density $k_{1}^{y}$ ) during the course of a vehicle searching and conducting a D-MLC, the maximum number of gaps $\left(N^{\prime}\right)$ that a vehicle can meets is estimated by Equation (33) below. 


$$
N^{\prime}=\frac{L_{y}}{v_{2}^{y^{\prime}}}\left(v_{1}^{y}-v_{2}^{y^{\prime}}\right) k_{1}^{y}
$$

Where, traffic flow speed $v_{1}^{y}$ and density $k_{1}^{y}$ can be calculated according to Equations (20) and (28).

In general, a leading vehicle will try $n$ gaps in a length of $L_{y}^{\prime}\left(n \leq N^{\prime}\right.$ and $\left.L_{y}^{\prime} \leq L_{y}\right)$ to complete a successful D-MLC (i.e. the successful lane change happens at the $n^{\text {th }}$ gap). Clearly, the value of $n$ is proportional to $L_{y}^{\prime}$, given the traffic density on the target lane, Lane 1, is uniform. Then $L_{y}^{\prime}$ can be estimated by Equation (34) below.

$$
L_{y}^{\prime}=\frac{n}{N^{\prime}} L_{y}=\frac{n v_{2}^{y \prime}}{\left(v_{1}^{y}-v_{2}^{y \prime}\right) k_{1}^{y}}
$$

It is noticed that the only uncertain element to capture the value of $t_{y f}$ is the value of $n$. Assuming each attempt of a D-MLC maneuver will success with an independent probability $p, n$ follows a geometric distribution in general. However, given a D-MLC maneuver successes in the yellow zone, it is noticed that $n \leq N^{\prime}$. Thus, the expected value of (n|n $\left.\leq \mathrm{N}^{\prime}\right)$ is given in Equation (35) below.

$$
E\left(n \mid n<N^{\prime}\right)=\sum n p^{\prime}(n)=\sum_{n=1}^{N^{\prime}} n \frac{(1-p)^{n-1} p}{\sum_{n=1}^{N^{\prime}}(1-p)^{n-1} p}=\left[\frac{1}{p}-\frac{N^{\prime}(1-p)^{N^{\prime}}}{1-(1-p)^{N^{\prime}}}\right]
$$

Where, $p^{\prime}(n)$ is the condition probability that a vehicle successfully changes lane at the nth gap given $\mathrm{n} \leq \mathrm{N}^{\prime}$, i.e., the vehicle completes the lane change in the yellow zone; recall that $p$ is the probability that a safe lane change successes at each gap given in Equation (1).

Combining Equations (32)-(35), we obtain the expected time delay for the leading vehicle and all the affected following vehicles in a D-MLC group in Equation (36).

$$
E\left(t_{y f}\right)=\Lambda \mathrm{E}\left(n \mid n<N^{\prime}\right), \Lambda=\left(v_{2}^{i n}-v_{2}^{y^{\prime}}\right) L_{y} / N^{\prime} v_{2}^{i n} v_{2}^{y_{\prime}^{\prime}}
$$

Furthermore, it is recognized that not all the following vehicles in a D-MLC group will experience the same time delay to the leading vehicle, resulting from the forward shock wave. See the exception in Figure 5. A forward shock wave may reach the end of the yellow zone before it affects some following vehicles in a group. This situation happens when the distribution of the leading vehicles with D-MLC intention is relatively sparse and the corresponding D-MLC group is very large, including a long queue of the following vehicles in each D-MLC group. Hence, this study considers to scale the expected time delay $E\left(t_{y f}\right)$ in Equation (36) by the proportion of the following vehicles which are actually affected by a forward shock wave.

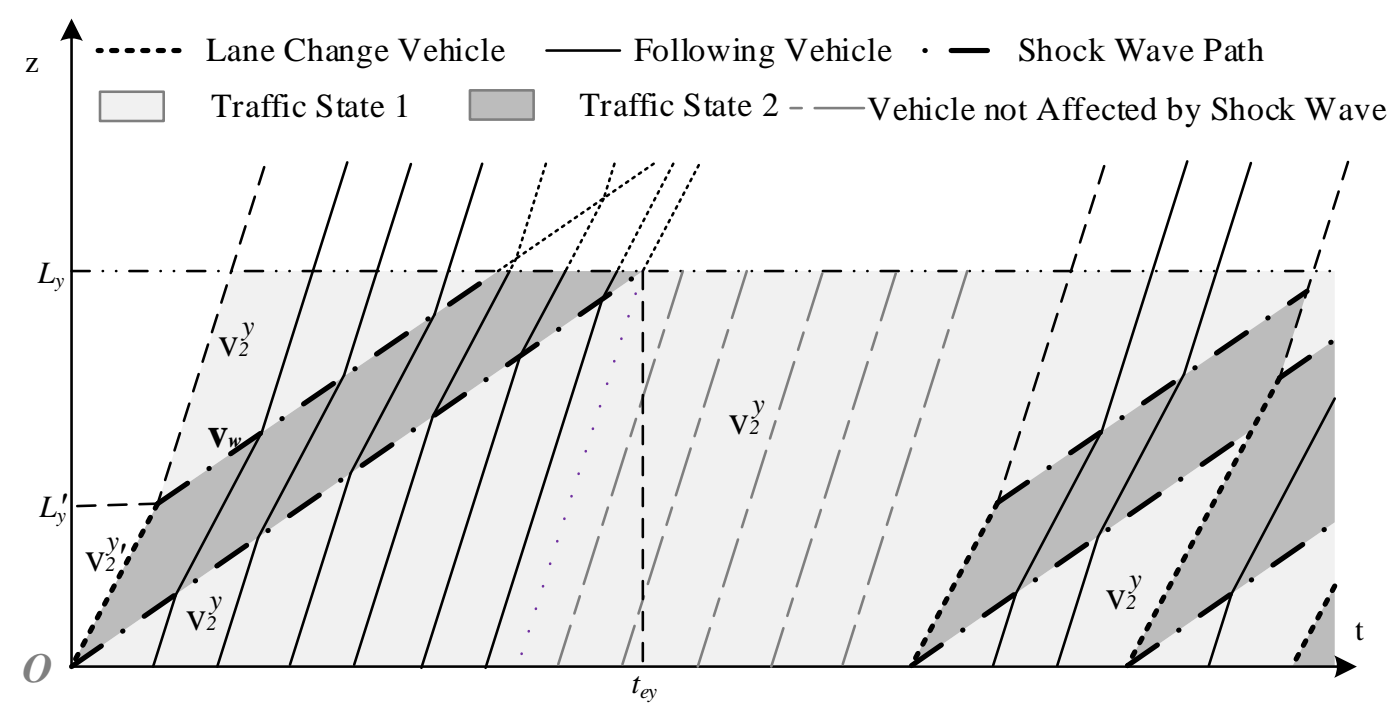

Figure 5. Forward shock wave path in a traffic with low density of vehicles with D-MLC intention

To do this, we first investigate the D-MLC group (denoted as $\mathrm{G}^{*}$ ), in which all the following vehicles are affected by a shock wave just before the last vehicle in $\mathrm{G}^{*}$ reaches to the boundary of the yellow zone (i.e. the point $\left(L_{y}, t_{e y}\right)$ in Figure 5. Then this study introduces another value $m^{*}$, which represents the maximum number of the following vehicles that are affected by a forward shock wave generated by the leading vehicle in $\mathrm{G}^{*}$. According to the geometry relationship given in Figure 5, 
Equation (37) provides the estimation of $m^{*}$.

$$
m^{*}=\frac{L_{y}}{v_{w}}\left(v_{2}^{y}-v_{w}\right) k_{2}^{y}
$$

Where, $\frac{L_{y}}{v_{w}}\left(v_{2}^{y}-v_{w}\right)$ represents the relative movement between the shock wave and the traffic flow during the course of the shock wave goes through the yellow zone; multiplying it with the traffic density $k_{2}^{y}$ gives us the value of $m^{*}$. Note that the average traffic density $\left(k_{2}^{y}\right)$ and initial speed $\left(v_{2}^{y}\right)$ on the subject lane (Lane 2) in the yellow zone are used to represent the traffic flow state.

Denoting $m_{a f}$ as the number of the following vehicles which are affected by a forward shock wave, we have $m_{a f}=m$ if $m \leq m^{*}$; and $m_{a f}=m^{*}$ if $m>m^{*}$. Recall that $m$ is considered as a random variable which follows geometric distribution with the probability function $p(m)=\beta(1-$ $\beta)^{m-1} . E(m)=\sum_{m=1}^{+\infty} m \beta(1-\beta)^{m-1}$. Accordingly, the expected number of vehicles which are affected by a shock wave is $E\left(m_{a f}\right)=\sum_{m=1}^{m^{*}} m \beta(1-\beta)^{m-1}+m^{*} \sum_{m=m^{*}}^{+\infty} \beta(1-\beta)^{m-1}$. Hence, Equation (38) below provides the scaled expected time delay experienced by an affected following vehicle in a D-MLC group on Lane 2.

$$
\begin{aligned}
& \mathrm{T}_{2}^{y f}=\frac{E\left(m_{a f}\right)}{E(m)} E\left(t_{y f}\right) \\
& =\frac{\sum_{1}^{m^{*}} m \beta(1-\beta)^{m-1}+m^{*} \sum_{m^{*}}^{+\infty} \beta(1-\beta)^{m-1}}{\sum_{1}^{+\infty} m \beta(1-\beta)^{m-1}} E\left(t_{y f}\right) \\
& =\Lambda\left[\frac{1}{p}-\frac{N^{\prime}(1-p)^{N^{\prime}}}{1-(1-p)^{N^{\prime}}}\right]\left[1-(1-\beta)^{m^{*}}\right],
\end{aligned}
$$

\subsubsection{Time delay on Lane 2 due to the backward shock wave}

This section studies the effect of a backward shock wave generated by a D-MLC in the yellow zone. Given the assumption (I), a vehicle which does not complete its lane change intention in the green zone will conduct a D-MLC maneuver immediately after it enters the yellow zone. The generated backward shock wave will affect vehicles on Lane 2 in the green zone or even further upstream traffic. In the other words, the traffic flow in the green zone and its upstream will be co-affected by both S-MLC and DMLC maneuvers. The influence of the S-MLC maneuvers has been discussed in previous section. Here, we focus on the impact of the D-MLC maneuvers. Figure 6 presents our analysis of the backward shock waves. Specifically, when a vehicle with lane change intention enters to the yellow zone at the start point $\mathrm{O}$, it starts a D-MLC searching with a slow speed $v_{2}^{y \prime}$ and successes at point A, where the following vehicles restore their speed to original speed $v_{2}^{y}$, which is the average speed on Lane 2 in the green zone and can be calculated by Equation (20). Accordingly, this D-MLC maneuver will produce two shock waves: OB and AC. In reality, both of them may propagate backward across the intersection of the studied road segment and cause extra delay on its adjacent roads. This study only counts the delay of the vehicles on this road segment and ignores this extra delay due to the uncertainty of traffic flow at the intersections and adjacent roads. Moreover, Figure 6 indicates that each vehicle may experience several shock waves. Hence, the total delay experienced by a vehicle can be estimated by the product $N_{s w} E\left(t_{y b}\right)$, where $N_{s w}$ is the expected number of shock waves and $E\left(t_{y b}\right)$ is the expected time delay caused by each backward shock wave.

Along with this thought, we first present our method to estimate $N_{s w}$. It is observed that all following vehicles in one D-MLC group will run a uniform distance of $l_{s w}$ between two consecutive shock waves, assuming they all follow the constant speed $v_{2}^{y}$ to enter the yellow zone, and then slow down to the same speed $v_{2}^{y \prime}$ due to D-MLC maneuvers. Considering the average spacing between two consecutive D-MLC leading vehicls is $L_{s p}=\frac{m}{k_{2}^{g}\left(L_{g}\right)}$, we estimate the average value of $l_{s w}$ in Equation (39), according to the geometry relationship shown by the shadowed triangle in Figure 6.

$$
l_{s w}=\frac{v_{w}}{v_{w}+v_{2}^{g}} L_{s p}=\frac{v_{w}}{v_{w}+v_{2}^{g}} \frac{m}{k_{2}^{g}\left(L_{g}\right)}=\frac{m\left(v_{f}-v_{2}^{y}-v_{2}^{y^{\prime}}\right)}{k_{2}^{g}\left(L_{g}\right)\left(v_{f}-v_{2}^{y^{\prime}}\right)},
$$

where $k_{2}^{g}\left(L_{g}\right)$ represents the traffic density at the end point in the green zone on Lane 2. It can be calculated by Equation (19). This study thinks $k_{2}^{g}\left(L_{g}\right)$ is a reasonably good approximation to the 
density around the start area of the yellow zone. Since the backward shock will spread to the end of the road segment, then it will affect the area in $L_{S}=L_{M A X}-L_{y}$. Accordingly, we estimate $N_{s w}$ through Equation (40) below.

$$
N_{s w}=\frac{L_{s}}{l_{s w}}=\frac{L_{s} \beta k_{2}^{g}\left(L_{g}\right)\left(v_{f}-v_{2}^{y^{\prime}}\right)}{v_{f}-v_{2}^{y}-v_{2}^{y \prime}}
$$

Where, $m$ is estimated by its mean $1 / \beta$.

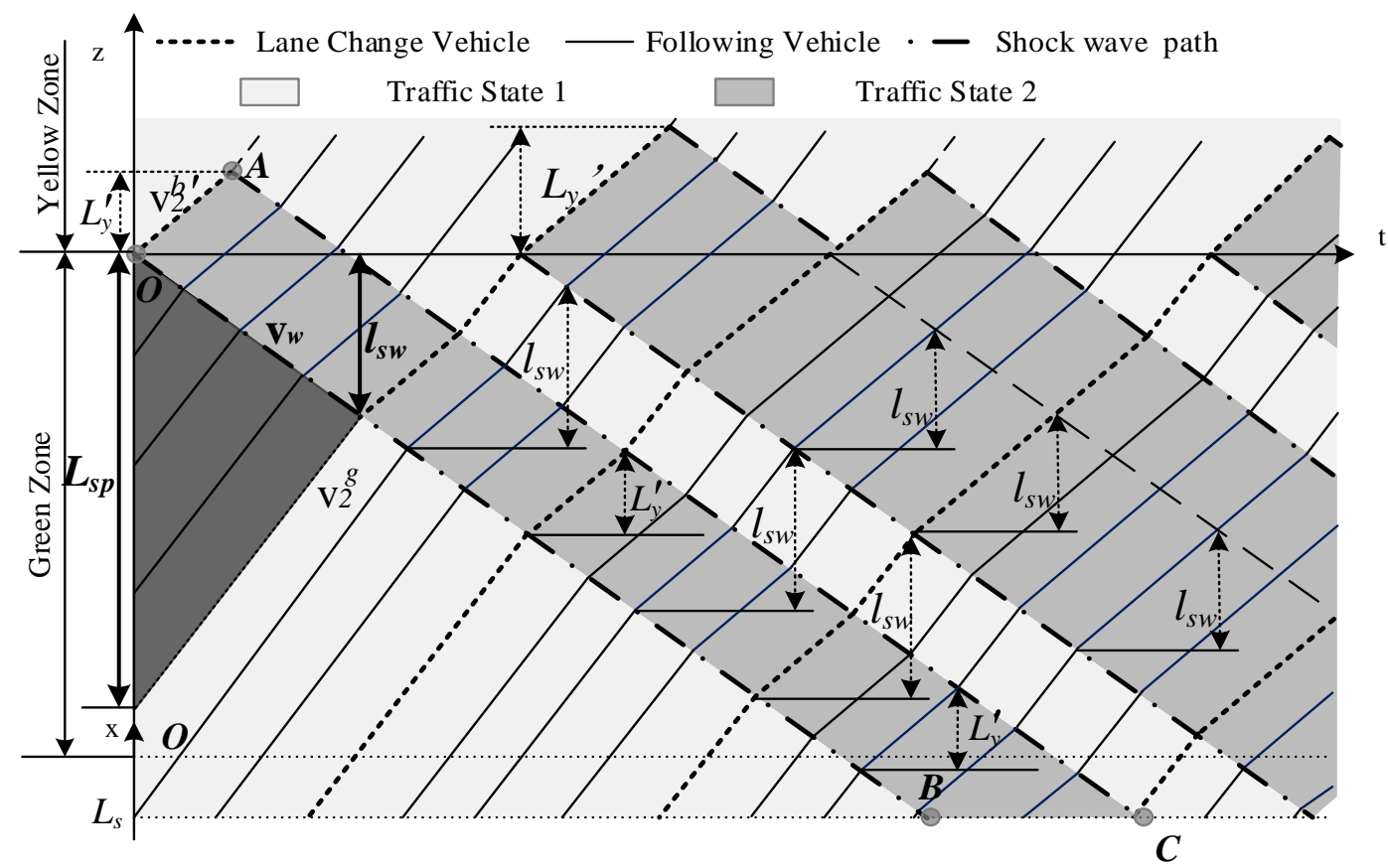

Figure 6. Illustration of backward shock wave

We next present our approach to approximate $E\left(t_{y b}\right)$. To do this, we observe another distance $L_{y}^{\prime}$ in Figure 6. $L_{y}^{\prime}$ is the length that a leading vehicle runs during the time period that it completes a successful D-MLC. Assuming that each vehicle conducting a D-MLC always decreases to the same speed $v_{2}^{y \prime}$ and their following vehicles always restore to the original $v_{2}^{y}$ (i.e. OB and AC are parallel in Figure 6), this study obtains that all vehicles affected by this shock wave will run the same distance during the time period. In addition, if there is no overlap between two consecutive shock waves, we have $L_{y}^{\prime} \leq l_{s w}$, otherwise, a vehicle will keep running through two shock waves in a low speed, then we have $L_{y}^{\prime} \geq l_{s w}$. Accordingly, $L_{y}^{\prime}$ and $l_{s w}$ are used to calculate the time delay under these two situations in Equations (41.1) and (41.2).

$$
t_{y b}=\left\{\begin{array}{l}
\frac{L_{y}^{\prime}}{v_{2}^{b \prime}}-\frac{L_{y}^{\prime}}{v_{2}^{i n}}, \quad \text { if } L_{y}^{\prime} \leq l_{s w} \\
\frac{l_{s w}}{v_{2}^{b^{\prime}}}-\frac{l_{s w}}{v_{2}^{i n}}, \quad \text { if } L_{y}^{\prime}>l_{s w}
\end{array}\right.
$$

In Equations (41.1) and (41.2), both $v_{2}^{y \prime}$ and $v_{2}^{\text {in }}$ are constant, but Equations (34) and (39) indicate that $L_{y}^{\prime}$ is a function of random variable $n$ (see its distribution given in (35)), and $l_{s w}$ is a function of random variable $m$ (see its distribution given in Equation (30) ). It has been recognized that both of these two random variables follow a geomantic distribution. Considering $m$ and $n$ are independent, this study thus has the joint probability distribution of $(m, n)$ in Equation (42).

$$
\mathrm{f}(\mathrm{m}, \mathrm{n})=\frac{\beta(1-\beta)^{m-1} p(1-p)^{n-1}}{\sum_{n=1}^{N^{\prime}}(1-p)^{n-1} p}, m \in[1,+\infty), n \in\left[1, N^{\prime}\right]
$$

Equations (34) and (39) further indicates that $\left(L_{y}^{\prime} \geq l_{s w}\right)$ is equivalent to $(\mathrm{m} \leq \gamma \mathrm{n})$ and $\left(L_{y}^{\prime}<l_{s w}\right)$ is equivalent to $(\mathrm{m}>\gamma \mathrm{n})$, where $\gamma=\frac{k_{2}^{g}\left(L_{g}\right) v_{2}^{y^{\prime}}\left(v_{f}-v_{2}^{y^{\prime}}\right)}{k_{1}^{y}\left(v_{f}-v_{2}^{g}-v_{2}^{y^{\prime}}\right)\left(v_{1}^{y}-v_{2}^{y \prime}\right)}$. Combining Equations (41.1), (41.2) and 
(42), this study obtains Equation (43) to calculate $E\left(t_{y b}\right)$, which represents the time delay caused by a backward shock wave.

$$
\begin{aligned}
& E\left(t_{y b}\right)=\sum_{n} \sum_{m}\left(\frac{L_{y}^{\prime}}{v_{2}^{y_{\prime}}}-\frac{L_{y}^{\prime}}{v_{2}^{\text {in }}}\right) \mathrm{f}(m, n \mid \mathrm{m} \leq \gamma \mathrm{n})+\sum_{n} \sum_{m}\left(\frac{l_{s w}}{v_{2}^{y_{\prime}}}-\frac{l_{s w}}{v_{2}^{\text {in }}}\right) \mathrm{f}(m, n \mid \mathrm{m}>\gamma \mathrm{n}) \\
& =\left[\sum_{n=1}^{N^{\prime}} \sum_{m=1}^{m=\gamma n}\left(\frac{l_{s w}}{v_{2}^{y_{1}}}-\frac{l_{s w}}{v_{2}^{\text {in }}}\right) \mathrm{f}(\mathrm{m}, \mathrm{n})+\sum_{n=1}^{N^{\prime}} \sum_{m=\gamma n}^{+\infty}\left(\frac{L_{y}^{\prime}}{v_{2}^{y^{\prime}}}-\frac{L_{y}^{\prime}}{v_{2}^{\text {in }}}\right) \mathrm{f}(\mathrm{m}, \mathrm{n})\right]
\end{aligned}
$$

Last, combining Equations (40) and (43), we obtain Equation (44) to estimate the average traffic delay resulting from the backward shock waves generated by the D-MLC maneuvers in the yellow zone. Equation (44) is adoptive to both the leading vehicle and the following vehicle in a D-MLC group.

$$
\mathrm{T}_{2}^{y b}=N_{s w} E\left(t_{y}\right)=\frac{L_{s}\left(v_{2}^{i n}-v_{2}^{y \prime}\right)}{v_{2}^{i n} v_{2}^{y \prime}}\left\{1-\frac{p(1-\beta)^{\gamma}\left[1-(1-p)^{N^{\prime}}(1-\beta)^{N^{\prime} \gamma}\right]}{\left[1-(1-p)(1-\beta)^{\gamma}\right]\left[1-(1-p)^{N^{\prime}}\right]}\right\}
$$

\subsection{Time delay caused by the D-MLC maneuvers}

A D-MLC maneuver can also affect three types of the traffic flow in the yellow zone. Type I flow includes the vehicles which will run through Lane 1 in the yellow zone. Type I flow includes two group vehicles: (i) the flow which enters Lane 1 at the entrance; it is equal to $q_{1 \rightarrow 1}$; (ii) the flow which enters Lane 1 in the green zone; it is equal to $q_{2 \rightarrow 1}^{g}=\alpha F_{g}\left(L_{g}\right) q_{2}^{i n}$. Type I flow will experience the delay $\left(t_{1 \rightarrow 1}^{y}\right)$ due to the traffic evolution on Lane 1 resulting from the D-MLC maneuvers in the yellow zone. Equation (29) presents the formulation to calculate the time delay, $t_{1 \rightarrow 1}^{y}$.

Type II flow includes the vehicles which go through Lane 2 in the yellow zone. It is the same to the flow $q_{2 \rightarrow 2}$ discussed for the Type II flow in the green zone, i.e., $q_{2 \rightarrow 2}=(1-\alpha) q_{2}^{\text {in }}+(1-$ $\left.F_{g}\left(L_{g}\right)\right)\left(1-F_{y}\left(L_{y}\right)\right) q_{2}^{i n}$. Type II flow will experience the time delay $\left(t_{2 \rightarrow 2}^{y}\right)$ caused by the shock waves generated by the D-MLC maneuvers in the yellow zone. Equations (38) and (44) will calculate $t_{2 \rightarrow 2}^{y}$ corresponding to the free flow or congested flow in the yellow zone.

Type III flow, denoted as $q_{2 \rightarrow 1}^{y}$, includes the vehicles which conduct D-MLC maneuvers in the yellow zone, i.e., $q_{2 \rightarrow 1}^{y}=F_{y}\left(L_{y}\right)\left(1-F_{g}\left(L_{g}\right)\right) \alpha q_{2}^{i n}$. The time delay of Type II flow can be calculated by Equation (45) below, given a D-MLC is conducted at the location $r \in\left[0, L_{y}\right]$.

$$
T_{2 \rightarrow 1}^{y}= \begin{cases}\int_{0}^{r}\left(\frac{d z}{\mathrm{v}_{2}^{y^{\prime}}}-\frac{d z}{\mathrm{v}_{2}^{i n}}\right)+\int_{r}^{L_{y}}\left(\frac{d z}{\mathrm{v}_{1}^{y}(z)}-\frac{d z}{\mathrm{v}_{2}^{i n}}\right) & \text { Forward shock wave } \\ \int_{0}^{r}\left(\frac{d z}{\mathrm{v}_{2}^{y^{\prime}}}-\frac{d z}{\mathrm{v}_{2}^{i n}}\right)+\int_{r}^{L_{y}}\left(\frac{d z}{\mathrm{v}_{1}^{y}(z)}-\frac{d z}{\mathrm{v}_{2}^{i n}}\right)+t_{2 \rightarrow 2}^{y} & \text { Backward shock wave }\end{cases}
$$

Under the forward shock wave, the first part of Equation (45) indicates the time delay caused by the deceleration of a vehicle to conduct a D-MLC, and the second part is the time delay due to traffic state evolution on Lane 1, i.e., Lane 1 will become denser since vehicles gradually switch to Lane 1 by the D-MLC maneuvers in the yellow zone. Under backward shock wave, the extra time delay $t_{2 \rightarrow 2}^{y}$ before they enter the yellow zone (i.e. in the green zone) is added.

Similar to the analysis used in the green zone, the location of a D-MLC (i.e., $r$ ) will affect the value of the time delay. Considering $r$ as a random variable with probability density function given in Equation (8), the time delay is calculated by Equations (46).

$$
\begin{aligned}
& t_{2 \rightarrow 1}^{y}=E\left(T_{2 \rightarrow 1}^{y}\right)=\int_{0}^{L_{y}} T_{2 \rightarrow 1}^{y} p_{y}(r) d r=\left.T_{2 \rightarrow 1}^{y} F_{y}(r)\right|_{0} ^{L_{y}}-\int_{0}^{L_{y}} F_{y}(r) d T_{2 \rightarrow 1}^{y} \\
& =\left.T_{2 \rightarrow 1}^{y} F_{y}(r)\right|_{0} ^{L_{y}}-\int_{0}^{L_{y}} F_{y}(r)\left(\frac{1}{v_{2}^{y^{\prime}}}-\frac{1}{\mathrm{v}_{1}^{y}(r)}\right) d r
\end{aligned}
$$

Last, Equation (47) calculates the average vehicle time delay resulting from the D-MLC maneuvers in the yellow zone. 


$$
E\left(T_{y}\right)=t_{1 \rightarrow 1}^{y}\left(\frac{q_{1 \rightarrow 1}+q_{2 \rightarrow 1}^{g}}{q_{1}^{i n}+q_{2}^{i n}}\right)+t_{2 \rightarrow 2}^{y}\left(\frac{q_{2 \rightarrow 2}}{q_{1}^{i n}+q_{2}^{i n}}\right)+t_{2 \rightarrow 1}^{y}\left(\frac{q_{2 \rightarrow 1}^{y}}{q_{1}^{\text {in }}+q_{2}^{i n}}\right)
$$

Appendix B presents the closed-form formulations for $t_{1 \rightarrow 1}^{y}$, and $t_{2 \rightarrow 1}^{y}$. Specifically, $t_{1 \rightarrow 1}^{y}$ is calculated in Equations (62) and (63) as Lane 1 is under free flow and congested flow conditions, respectively. $t_{2 \rightarrow 1}^{y}$ is calculated in Equation (64) under the condition that Lane 1 is under free flow and Lane 2 is under forward shock wave. $t_{2 \rightarrow 1}^{y}$ is calculated in Equation (65) under the condition that Lane 1 is under congestion flow and Lane 2 is under forward shock wave. $t_{2 \rightarrow 1}^{y}$ is calculated in Equation (66) under the condition that Lane 1 is under free flow and Lane 2 is under backward shock wave. $t_{2 \rightarrow 1}^{y}$ is calculated in Equation (67) under the condition that Lane 1 is under congestion flow and Lane 2 is under backward shock wave. $t_{2 \rightarrow 2}^{y}$ is calculated from shock wave analysis which does not need integration.

\subsection{Optimization model to identify the green zone and solution method}

$$
\begin{aligned}
& \text { OPT-II } \min \quad \mathrm{Z}=\left(t_{1 \rightarrow 1}^{g}+t_{1 \rightarrow 1}^{y}\right)\left(\frac{q_{1 \rightarrow 1}}{q_{1}^{\text {in }}+q_{2}^{i n}}\right)+\left(t_{2 \rightarrow 2}^{g}+t_{2 \rightarrow 2}^{y}\right)\left(\frac{q_{2 \rightarrow 2}}{q_{1}^{\text {in }}+q_{2}^{\text {in }}}\right) \\
& +\left(t_{2 \rightarrow 1}^{g}+t_{1 \rightarrow 1}^{y}\right)\left(\frac{q_{2 \rightarrow 1}^{g}}{q_{1}^{\text {in }}+q_{2}^{\text {in }}}\right)+\left(t_{2 \rightarrow 2}^{g}+t_{2 \rightarrow 1}^{y}\right)\left(\frac{q_{2 \rightarrow 1}^{y}}{q_{1}^{\text {in }}+q_{2}^{\text {in }}}\right) \\
& \text { s.t. } \quad 0 \leq \mathrm{L}_{g}+\mathrm{L}_{y} \leq \mathrm{L}_{\text {Max }} \\
& \mathrm{L}_{g}, \mathrm{~L}_{y} \geq 0 \\
& 0 \leq 1-(1-\mathrm{p})^{\frac{\mathrm{L}_{g}}{v_{2}}\left|v_{2}-v_{1}\right| k_{1}+1,} \leq 1 \\
& 0 \leq 1-(1-\mathrm{p})^{\frac{\mathrm{L}_{y}}{v_{2}}}\left|v_{2}^{y^{\prime}}-v_{1}\right| k_{1}, \leq 1 \\
& \sigma_{o}=\mathrm{F}_{g}\left(\mathrm{~L}_{g}\right)+\left(1-\mathrm{F}_{g}\left(\mathrm{~L}_{g}\right)\right) \mathrm{F}_{y}\left(\mathrm{~L}_{y}\right) \geq \sigma
\end{aligned}
$$

Plugging Equations (25) and (47) about the time delay that a vehicle experiences in the green and yellow zone, and Equations (7) and (8) that the lane change opportunities for a S-MLC and D-MLC in the green and yellow zones respectively, we will have the optimization model OPT-II. Again, Equation (48) indicates that all of the flow entering this highway segment will be affected by both S-MLC and D-MLC maneuvers, which lead to the respective gradual traffic evolution and shock waves. More exactly, the traffic flow going through Lane 1 without lane changes (i.e. $q_{1 \rightarrow 1}$ ) will experience the time delay $t_{1 \rightarrow 1}^{g}$ resulting from S-MLC maneuvers in the green zone, and the time delay $t_{1 \rightarrow 1}^{y}$ caused by D-MLC maneuvers in the yellow zone. The traffic flow going through Lane 2 without lane changes (i.e. $q_{2 \rightarrow 2}$ ) will experience the time delay $t_{2 \rightarrow 2}^{g}$ caused by S-MLC maneuvers and the time delay $t_{2 \rightarrow 2}^{y}$ caused by D-MLC maneuvers. The traffic flow which conducts S-MLC maneuvers in the green zone (i.e. $q_{2 \rightarrow 1}^{g}$ ) will experience the time delay $t_{2 \rightarrow 1}^{g}$ caused by S-MLC maneuvers and the time delay $t_{1 \rightarrow 1}^{y}$ caused by D-MLC maneuvers. The traffic flow which completes D-MLC maneuvers in the yellow zone (i.e. $q_{2 \rightarrow 1}^{y}$ ) will experience the time delay $t_{2 \rightarrow 2}^{g}$ caused by S-MLC maneuvers and the time delay $t_{2 \rightarrow 1}^{y}$ caused by D-MLC maneuvers.

Note that the relaxation phenomenon (Laval and Leclercq, 2008; Zheng et al., 2013), usually happening in a non-equilibrium lane change maneuvers ${ }^{5}$, is not considered by this study in developing the time delay formulation of the objective function for the main reasons below. Firstly, existing research on the relaxation phenomenon indicates that there is not a close relationship between the relaxation phenomenon and the occurrence location of such lane change maneuvers. In other words, wherever a non-equilibrium lane change happens, the relaxation phenomenon exists and causes certain time delay to the surrounding traffic. Therefore, the total traffic delay caused by relaxation phenomenon is mainly related to the volume of such lane change maneuvers rather than the location to perform them. Accordingly, the time delay resulting from relaxation phenomenon is almost a constant factor in the proposed optimization model. Then its effect does not significantly affect the choice of the location of advanced warning in the proposed study. This observation is also verified by the experimental analysis in Section 4.2.3. Secondly, the analysis of traffic state evolution resulting from smooth lane change

\footnotetext{
${ }^{5}$ Non-equilibrium: A lane change when the spacing between lane changer and its leader and /or its follower much smaller than the fundamental diagram spacing (Leclercq et al., 2007)
} 
maneuvers (see discussion in Section 3.2) partially covers the effect of the relaxation phenomenon (slowing down the average speed of surrounding traffic) from the macroscopic view. Ignoring the microscope process of relaxation phenomenon helps simplify the formulation of time delay in the proposed optimization model, and hence balances model accuracy and tractability. Thirdly, the research of Leclercq et al. (2007) indicated that most non-equilibrium lane change happened on on-ramp direction (from shoulder lane to center lane) rather than on off-ramp direction (from center lane to shoulder lane), and so does the relaxation phenomenon. Our experiments in Section 4.2.3 indicate this observation too.

Overall, this study thinks that even though the relaxation phenomenon is critical in studying the lane change impact in general, it does not significantly affect the solution of the proposed optimization model, which seeks to search the optimal location of advance warning nearby a highway off-ramp. To balance mathematical tractability and traffic realties, this study ignores the effect of the relaxation phenomenon. Our experiments in Section 4 indicate that this simplification indeed leads to acceptable errors in the solution.

It is noticed that OPT-II model only includes two decision variables $L_{g}$ and $L_{y}$, i.e., the lengths of the green zone and the yellow zone. This study thus uses the grid search method to search for the optimal solution. The grid search procedure are presented as follows.

1. Input traffic data, such as $q_{1}^{i n}, q_{2}^{i n}, v_{1}^{\text {in }}$ and $v_{2}^{\text {in }}$ and set the parameters such as $L_{M a x}$ and $\sigma$. Initialization $L_{g}=0$. Set the initial minimization time delay $Z_{\text {Min }}$ as infinity. Set step size, $\Delta L_{g}=1, \Delta L_{y}=1$ (meter).

2. While $L_{g} \leq L_{\text {Max }}$

1) Initialization $L_{y}=0$

2) While $L_{y} \leq L_{\operatorname{Max}}$ If $\left(L_{g}, L_{y}\right)$ satisfies all constrains, compute the time delay $Z$.

If the computed time delay $Z$ is smaller than $Z_{\text {Min }}$, update $Z_{\text {Min }}=Z$. Record the location, $L_{g, \text { Min }}=L_{g}, L_{y, \text { Min }}=L_{y}$. Else, do nothing.

Else, do noting

Update $L_{y}=L_{y}+\Delta L_{y}$. And go back to step 2).

3) Update $L_{g}=L_{g}+\Delta L_{g}$. And go back to step 2 .

3. Output optimization solution, $Z_{\text {Min }}, L_{g, \text { Min }}$, and $L_{y, \text { Min }}$

\section{Numerical Experiments}

\subsection{Experiment setup and design}

This study conducts the numerical experiments to demonstrate the performance of our approaches and explore insights. More specifically, we will validate the optimality of the lane change guidance provided by the OPT-II. We expect that if most of vehicles start their lane change intention at the beginning of the green zone, then most of them can complete smooth lane changes in the green zone, and the rest small portion of vehicles can finish lane changes in the yellow zone. More importantly, these lane change maneuvers lead to minimal negative impact on the fleet traffic delay.

The setup of the experiments is specified as follows. (i) All the experiments are conducted on a two-lane road segment developed in Paramics 6.9.3. As shown in Figure 7, the highway segment has one entrance and an exit. An off-ramp is located at the end of the right hand side lane (Lane 1). The length of the road segment before the off-ramp is $2.2 \mathrm{~km}$. The speed limit for main traffic (Lane 1 and Lane 2) and off-ramp traffic is $95 \mathrm{~km} / \mathrm{h}$ and $45 \mathrm{~km} / \mathrm{h}$ respectively. The capacity for the main road is 2400 vehicle per hour per lane. (ii) A advance warning for the off-ramp is located at the distance of $w$ upstream to the off-ramp. In the simulation model, vehicles are only permitted to perform lane changes after they pass the location of the advance warning. (iii) The proposed experiments are conducted under different traffic regimes ranging from LOS A to LOS F (according to HCM 2010), which correspond to the traffic flow changing from $2500 \mathrm{vph}$ to $5000 \mathrm{vph}$ with step size $500 \mathrm{vph}$. Under each traffic flow scenario, $20 \%$ of the total traffic flow needs to merge to the off-ramp lane, i.e., $(\alpha=20 \%)$. It is expected that the optimal advance warning will ensure $99 \%$ of them to success in the suggested the green and the yellow zones. Thus, the threshold $\sigma$ used in Equation (53) of the OPT is set as 99\%. (iv) The grid 
search algorithm for solving the OPT model is implemented by MATLAB 7.1 and run on a computer with processor: Intel(R) Core(TM) i7-3770 CPU @ 3.40GHz and RAM: 8.0 GB.

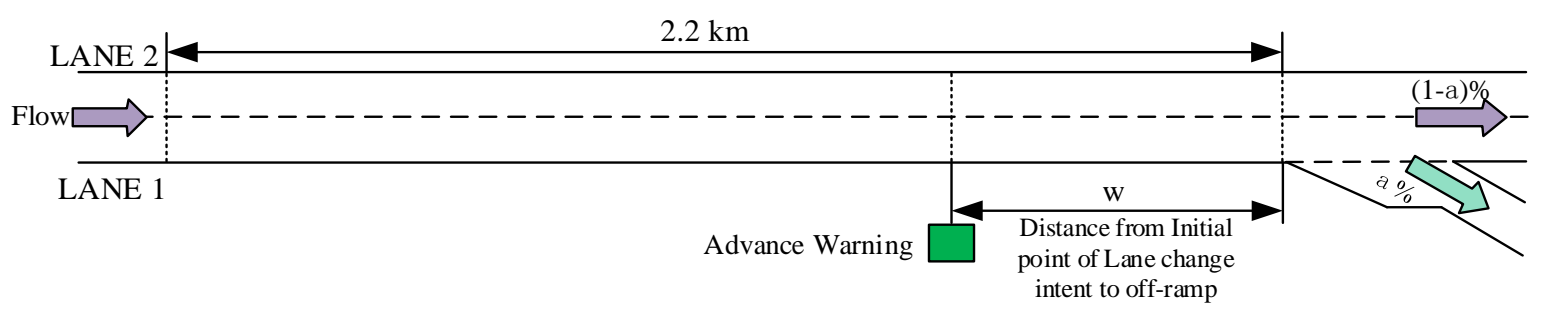

Figure 7. The highway segment model in the simulation

\subsection{Experimental and Results}

4.2.1 Validation of the green and yellow zones obtained from the OPT

This study first exams if the green and yellow zones obtained from the solution of the OPT model exist in the traffic flow, corresponding to the advance warning set at the beginning of the green zone. This validation is conducted with several experimental steps below.

Step 1, Run the OPT model as traffic lanes under different traffic flow regimes from LOS A to LOS F. Table 1 presents the lane change zones of the OPT from LOS A to LOS F. We give more comprehensive discussion of the green and yellow zone distribution here. (i) Under very sparse traffic, such as LOS A-B, the OPT solution suggests locating the advance warning at the place almost right before the off-ramp $\left(L_{g}=0 ; L_{y}=0\right)$, which won't lead significant traffic time delay $\left(T_{o}=0 \mathrm{~s}\right)$. As a result, both the green and yellow zones downstream of the advance warning vanish. In other words, vehicle may conduct MLC at any location upstream to the off-ramp without interrupting traffic and a particular advance warning is not necessary. (ii) Under free traffic low, such as LOS C, the optimal solution of the OPT model suggests setting the advance warning at the location of $\left(L_{g}=212 ; L_{y}=0\right)$, which only leads to the green zone in the downstream of the advance waning. (iii) Under mild traffic congestion, such as LOS D-E, the optimal solution of the OPT model suggests locating the advance warning, which makes the green and yellow zones coexist in the downstream of the advance warning. Moreover, as traffic becomes more and more congested, the location of the advance warning moves further away from the off-ramp (see $w=499$ under LOS D and $w=977$ under LOS E).

Table 1. The optimal location of the green and yellow zones, and the advance warning from the OPT

\begin{tabular}{|c|c|c|c|c|}
\hline \multicolumn{2}{|c|}{ Traffic condition } & \multicolumn{2}{|c|}{ Lane change zone } & $\begin{array}{c}\text { Location of the advance warning } \\
w=L_{y}+L_{g}\end{array}$ \\
\hline Flow & LOS & $L_{g}$ & $L_{y}$ & 0 \\
\hline$<2000$ & A & 0 & 0 & 0 \\
\hline 2000 & B & 0 & 0 & 212 \\
\hline 2500 & C & 212 & 0 & 499 \\
\hline 3000 & D & 319 & 180 & 643 \\
\hline 3500 & D & 440 & 203 & 977 \\
\hline 4000 & E & 639 & 338 & 2200 \\
\hline 4500 & E & 1635 & 565 & 2200 \\
\hline 5000 & F & 1231 & 969 & \\
\hline
\end{tabular}

Step 2, Implements the optimal location of the advance warning into the simulation model through locating the advance warning of the off-ramp to the beginning of the green zone, i.e. $w=L_{g}+L_{y}$ in Table 1. Then, we run the simulation model under corresponding traffic flow scenarios.

Step 3, Analysis the lane change performance in the green and yellow zones in the simulation model and validate if they are consistent to the corresponding definitions for the green and yellow zones first given in the problem formulation in Section 2. Namely, we will exam if the green and yellow zones in the simulation grant enough lane change opportunities; moreover, the green zone protects enough smooth lane changes without speed deceleration. To do this, we compare the values of the three items obtained from the OPT model and the simulation under each traffic regime: (i) $\sigma$, successful lane 
change probability/percentages in the green and yellow zones, (ii) $F_{g}$, the successful lane change probability without deceleration in the green zone, (iii) $F_{y}$, the successful lane change probability/percentages with deceleration in the yellow zone. The results are discussed as follows.

Table 2 below presents the results about $\sigma$, where $\sigma_{s}$ and $\sigma_{o}$ represent the values from the simulation and the OPT model respectively. As we mentioned in the experiment setup, $\sigma_{o}$ in the OPT is $99 \%$. The results in Table 2 show that the value of $\sigma_{s}$ is greater than the threshold of $\sigma_{o}$ under each traffic regime. Thus, we claim that the green zone and yellow zone together grant enough lane change opportunity for vehicles to finish the MLC maneuvers in the simulation.

Table 2. Successful lane change probability in the green zone and yellow zone

\begin{tabular}{|c|c|c|c|}
\hline \multicolumn{2}{|c|}{ Traffic condition } & \multicolumn{2}{c|}{ Total successful lane change rate in the green and yellow zones } \\
\hline Flow & LOS & $\sigma_{o}$ & $\sigma_{s}$ \\
\hline$<2000$ & A & OPT model threshold & $100 \%$ \\
\hline 2000 & B & $\geq 99 \%$ & $100 \%$ \\
\hline 2500 & C & & $100 \%$ \\
\hline 3000 & D & & $99.90 \%$ \\
\hline 3500 & D & & $99.09 \%$ \\
\hline 4000 & E & & $99.11 \%$ \\
\hline 4500 & E & & $99.43 \%$ \\
\hline 5000 & F & & $99.32 \%$ \\
\hline
\end{tabular}

Table 3 below presents the results about $F_{g}$, where $F_{o, g}$ represents the probability to conduct SMLC in the green zone obtained from the OPT model; $F_{s, g, w / o}$ and $F_{s, g, w}$ represent the percentage of the vehicles which successfully conduct respective S-MLC and D-MLC maneuvers in the zone $\left[L_{y}, L_{g}+L_{y}\right]$ (considering the off-ramp as the origin; the $\mathrm{x}$-axis points to the upstream of the off-ramp) during the course of the simulation. The results in Table 3 show that both $F_{o, g}$ and $F_{s, g, w / o}$ vary from $99 \%$ to $69 \%$ as the traffic congestion changes from LOS C to LOS F. In addition, $F_{s, g, w / o}$ is reasonably close to $F_{o, g}$ under each traffic regime, even though the relative error increases with the traffic congestion level and ends at a value of $-7.27 \%$ at LOS F. These observation confirms two critical points under our expectation. (i) It confirms the consistency between our mathematical formulation and the simulation results. (ii) The green zone under each traffic regime provides sufficient opportunities for vehicles to conduct S-MLC maneuvers. Thus, we conclude that the zone $\left[L_{y}, L_{g}+L_{y}\right]$ in the simulation consists with the green zone identified by the OPT model.

Table 3 Successful lane change probability in the green zone

\begin{tabular}{|c|c|c|c|c|c|}
\hline \multirow{2}{*}{ Traffic condition } & \multicolumn{4}{|c|}{ Lane change success rate in the green zone } \\
\cline { 4 - 6 } & \multirow{2}{*}{$F_{o, g}$} & \multicolumn{3}{|c|}{ Simulation } \\
\cline { 4 - 6 } Flow & LOS & & $F_{s, g, w / o}$ & Relative error & $F_{s, g, w}$ \\
\hline 2500 & $\mathrm{C}$ & 0.99 & 0.9881 & $-0.19 \%$ & 0.0119 \\
\hline 3000 & $\mathrm{D}$ & 0.8988 & 0.8864 & $-1.38 \%$ & 0.1021 \\
\hline 3500 & $\mathrm{D}$ & 0.7862 & 0.7659 & $-2.58 \%$ & 0.1948 \\
\hline 4000 & $\mathrm{E}$ & 0.8089 & 0.7906 & $-2.26 \%$ & 0.1767 \\
\hline 4500 & $\mathrm{E}$ & 0.8224 & 0.7787 & $-5.31 \%$ & 0.1926 \\
\hline 5000 & $\mathrm{~F}$ & 0.7401 & 0.6863 & $-7.27 \%$ & 0.1853 \\
\hline
\end{tabular}

Moreover, it is noticed that the value of $F_{s, g, w / o}$ is always a little bit smaller than the value of $F_{o, g}$. After analyzing the trajectories of vehicles, it is found that D-MLC maneuvers also occur in the green zone in the simulation. As the traffic congestion level increases, this impact becomes more significant. Moreover, some lane change maneuvers with acceleration also happens. The existing of DMLC and LC with acceleration maneuvers in the green zone are not consistent to our assumption about the green zone to develop the mathematical formulations. Thus, it leads to the difference between 
$F_{s, g, w / o}$ and $F_{o, g}$. The good point is that the percentage of the vehicles which violate the assumption is not very high (about $1 \%$ under LOS C, and 19\% under LOS F). Thus, most of vehicles complete SMLC maneuvers in the green zone. Overall, the experimental results indicate that the OPT model can efficiently identify the green zone existing in the traffic flow, which protects most of vehicles to do SMLC under a given traffic condition, even though we cannot control the lane change behavior in the simulation.

Table 4 below presents the results about $F_{y}$, in which $F_{o, y}$ obtained from the OPT model represents the opportunity that a vehicle completes a D-MLC in the yellow zone; $F_{s, y, w}$ and $F_{s, y, w / o}$ represents the percentage of the vehicles which successfully conduct respective D-MLC and S-MLC maneuvers in the zone of $\left[0, L_{y}\right]$ (considering the off-ramp at the origin; the x-axis points to the upstream of the off-ramp) during the course of the simulation. They are calculated through statistic method according to vehicle trajectory data.

The results in Table 4 indicate that the value of $F_{o, y}$ is much larger than the actual percentage $F_{s, y, w}$ under each traffic regime. Through analyzing vehicle trajectory data, it is recognized that this big difference results from the D-MLC in the green zone. Because many vehicles already successfully compete lane changes in the green zone, only a few rest of vehicles need to complete lane change in the zone $\left[0, L_{y}\right]$. However, after examining all lane change maneuvers in the zone, this study found that most of successful lane change maneuvers (almost greater than $80 \%$ ) in the zone $\left[0, L_{y}\right]$ are D-MLC maneuvers. As the level of traffic congestion increases, the percentage of the D-MLC maneuvers becomes higher in this zone. This observation indicates that $\left[0, L_{y}\right]$ corresponds to the yellow zone identified by the OPT model.

Overall, the experimental results in Tables 1-4 demonstrate that if vehicles start to perform lane change after they pass the advance warning located at $w=L_{g}+L_{y}$ upstream to the off-ramp, the zone $[0, w]$ will protect sufficient opportunities for vehicles to successfully complete lane changes under different traffic congestion levels. Moreover, most of vehicles can complete S-MLC maneuvers in the green zone and the rest of vehicles will conduct the D-MLC maneuvers in the yellow zone. Thus, we confirm the existence and correctness of the green and yellow zones proposed by the OPT model.

Table 4 Successful lane change probability in the yellow zone

\begin{tabular}{|c|c|c|c|c|c|}
\hline \multirow{2}{*}{\multicolumn{2}{|c|}{ Traffic condition }} & \multicolumn{4}{|c|}{ Lane change success rate in the yellow zone } \\
\hline & & \multirow{2}{*}{$F_{o, y}$} & \multicolumn{3}{|c|}{ Simulation } \\
\hline Flow & LOS & & $F_{S, y, w}$ & $F_{s, y, w / o}$ & Percentage of with deceleration \\
\hline 2500 & $\mathrm{C}$ & 0 & 0 & 0 & 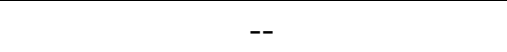 \\
\hline 3000 & $\mathrm{D}$ & 0.0912 & 0.00833 & 0.00217 & $79.37 \%$ \\
\hline 3500 & $\mathrm{D}$ & 0.2038 & 0.02434 & 0.00586 & $80.61 \%$ \\
\hline 4000 & $\mathrm{E}$ & 0.1811 & 0.0193 & 0.0045 & $81.09 \%$ \\
\hline 4500 & $\mathrm{E}$ & 0.1676 & 0.01978 & 0.00322 & $85.99 \%$ \\
\hline 5000 & $\mathrm{~F}$ & 0.2499 & 0.1113 & 0.0103 & $91.53 \%$ \\
\hline
\end{tabular}

4.2.2 Validate the optimality of the advance warning

This study next validates the optimality of the green and yellow zones (i.e. the location of the advance warning). We expect that the fleet traffic delay is minimized if most of the lane change occurs in the green and yellow zones downstream of the advance warning. To do this, our experiments need one more step: screening the fleet traffic flow delay through manually moving the location of the advance warning in the simulation model from $0 \mathrm{~m}$ to 2200 meters away from the off-ramp with step size 200 meters. The similar experiments are run under traffic regimes LOS A to LOS F. Figure 8 presents the variation of the time delay as the location of the advance warning was moved away from the off-ramp under each of the different traffic conditions. The point of the lowest delay on each curve represents the optimal location of the advance warning under the corresponding traffic condition. Then, the optimal location which results in the minimum traffic delay under each LOS is recorded. Note that the curves for the experiments with traffic flow less than $2500 \mathrm{vph}$ are not included since no traffic delay occurs no matter where the advance warning is put. 


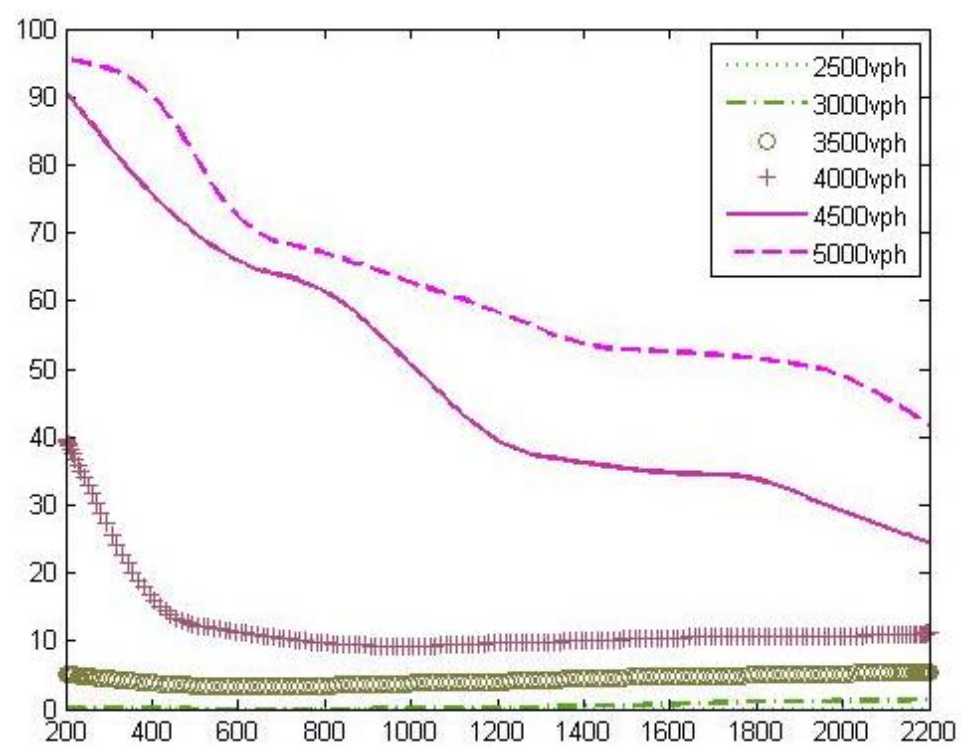

Figure 8 . Time delay from the simulation vs. the location of the advance warning

The experiments results are presented in Table 5, where $w_{s}$ and $w_{o}$ represent the optimal location of the advance warning found in the simulation and by the OPT respectively, and $T_{S}$ and $T_{o}$ represent the corresponding minimum time delays. Below highlights the key observations.

When the traffic flow is less than $2500 \mathrm{vph}$, traffic service is under LOS A or B the traffic delay in the simulation is not sensitive to the location of the advance warning. This is consistent to the optimal solution of the OPT model in Table 1 , which suggests $w_{0}=0$, meaning no advance warning is needed. When the traffic flow rate increases to $2500 \mathrm{vph} \sim 3000 \mathrm{vph}$, traffic service is in LOS C or LOS D, $w_{o}$ is in the optimal range of $w_{s}$ (LOS C: $w_{o}=212$ which is in the range of $w_{s}=(0,2200)$; LOS D: $w_{o}=449$ which is in the range of $\left.w_{s}=(200,1200)\right)$. When the traffic flow is above 3000vph, traffic service is in LOS E or LOS F, $w_{o}$ is consistent to $w_{s}$ very well under each traffic regime. Therefore, the experimental results indicate that $w_{S}$ and $w_{o}$ fit well under all traffic regimes. In addition, it is observed that as traffic becomes more congested, the advance warning should be further away from the off-ramp. At the meantime, it is noticed that the green zone moves away from the off-ramp. These observations are consistent to our intuition. Table 5 also indicates that $T_{S}$ and $T_{o}$ corresponding to each pair of $w_{S}$ and $w_{O}$ are close, and the relative errors are smaller than $2 \%$ as traffic flow varies from 2500 to $4000 \mathrm{vph}$. The relative errors increase to $9.43 \%$ and $4.72 \%$ as the traffic flow rates are 4500 and 5000 respectively. Thus, the minimum time delay $T_{o}$ of the OPT is consistent to the minimum time delay in the simulation. Note that, the time delay in table 5 is the average time delay for a vehicle in traffic flow.

Table 5 Optimal location of the advance warning and time delay

\begin{tabular}{|c|c|c|c|c|c|c|c|}
\hline \multicolumn{2}{|c|}{ Traffic condition } & \multicolumn{2}{|c|}{ The Location of Advance Warning } & \multicolumn{3}{c|}{ Time delay (s) } \\
\hline Flow & LOS & $w_{o}$ & $w_{S}$ & Relative error & $T_{o}$ & $T_{S}$ & Relative error \\
\hline$<2000$ & $\mathrm{~A}$ & 0 & $0-2200$ & 0 & 0 & 0 & -- \\
\hline 2000 & $\mathrm{~B}$ & 0 & $0-2200$ & 0 & 0 & 0 & -- \\
\hline 2500 & $\mathrm{C}$ & 212 & $0-2200$ & 0 & 0.0003 & 0 & -- \\
\hline 3000 & $\mathrm{D}$ & 499 & $200-1200$ & 0 & 0.297 & 0 & - \\
\hline 3500 & $\mathrm{E}$ & 643 & 600 & $7.17 \%$ & 3.0824 & 3.13224 & $-1.59 \%$ \\
\hline 4000 & $\mathrm{E}$ & 977 & 1000 & $-2.30 \%$ & 9.0759 & 9.17039 & $-1.03 \%$ \\
\hline 4500 & $\mathrm{~F}$ & 2200 & 2200 & 0 & 26.8014 & 24.4926 & $9.43 \%$ \\
\hline 5000 & $\mathrm{~F}$ & 2200 & 2200 & 0 & 39.7336 & 41.7035 & $-4.72 \%$ \\
\hline
\end{tabular}

These observations from Table 5 conclude that the OPT model is able to provide the optimal location to set the advance warning, which will guide the lane change maneuvers and lead to minimum traffic dely. Thus, this study successfully validate the optimality of the location of the advance warning (i.e., the green and yellow zones) obtained from the OPT, and demonstrates the efficiency of the proposed 
approach. Overall, the experimental experiments validate the efficiency of the proposed approaches. Note that we also above experiment on lane change rate $\alpha=10 \%$ condition. The result is similar. So we only present $\alpha=20 \%$ here.

\subsubsection{The effect on capacity drop and traffic oscillation}

To provide more comprehensive insights for the effect of the proposed approach, this study investigates the mitigation of capacity drop and traffic oscillation by placing the advance warning at the optimal location, even though they are not explicitly considered in this study.

To detect traffic capacity, the simulation model for new experiments put loop detectors on target lane and off-ramp just downstream to the bottleneck (see Figure 9), so that the two detectors will catch all traffic flow out of the bottleneck. To ensure flow reaching capacity can be observed, a large entering flow such as $5000 \mathrm{vph}$ is set and the flow capacity at both subject lane and target lane are set as $2400 \mathrm{vph}$. Next, the experiments collect traffic flow data at the loop detectors over the simulation running time (60 minutes after 30 minutes warming up) under ten scenarios, in which the advance warning in the simulation model was located at 200 to 3000 meters away from the off-ramp with step size 400 meters. Table 6 presents observed traffic capacity at the detected points and the corresponding capacity drop under different scenarios. It is noticed that at the advance warning is put at the optimal location, $2200 \mathrm{~m}$ away the off-ramp, the capacity drop reaches minimum value $8.00 \%$. The experiment showed that placing advance warning at optimal location can efficiently mitigate the capacity drop.

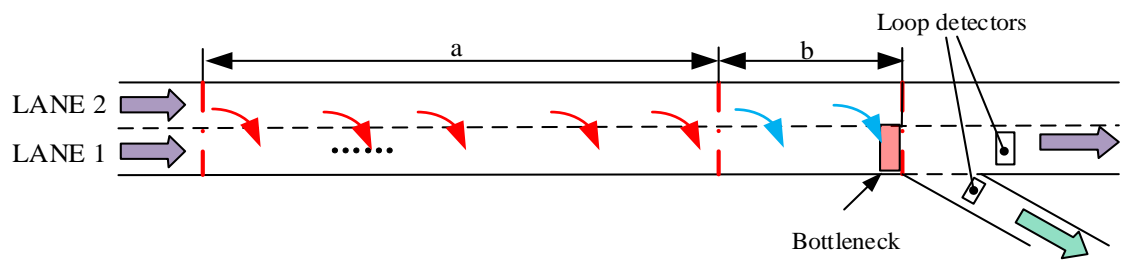

Figure 9. Loop detector set in capacity drop experiment

Table 6 Capacity drop as advance warning was given at different locations

\begin{tabular}{|c|c|c|c|}
\hline Location $(\mathrm{m})$ & Observed Capacity & Original Capacity & Capacity drop \\
\hline 200 & 1946.6 & 2400 & $-18.89 \%$ \\
\hline 600 & 2009 & 2400 & $-16.29 \%$ \\
\hline 1000 & 2124.6 & 2400 & $-11.48 \%$ \\
\hline 1400 & 2174.8 & 2400 & $-9.38 \%$ \\
\hline 1800 & 2193 & 2400 & $-8.63 \%$ \\
\hline 2200 & 2208 & 2400 & $-8.00 \%$ \\
\hline 2600 & 2206.8 & 2400 & $-8.05 \%$ \\
\hline 3000 & 2199 & 2400 & $-8.38 \%$ \\
\hline
\end{tabular}

To detect traffic oscillation, the new experiments set entering flow as $4000 \mathrm{vph}$, which leads to unstable traffic flow with LOS E. Thus, traffic flow oscillation is often triggered so that can be observed in the experiments. According to our previous experiments, the optimal advance warning location is $977 \mathrm{~m}$ upstream to the off-ramp. Four groups of loop detectors are setting on both Lane 1 and Lane 2 to collect the aggregated speed data in every $5 \mathrm{~s}$ at $2000 \mathrm{~m}$ (D1), 1500m (D2), 1000m (D3) and 500m (D4) upstream to the off-ramp (see Figure 10). The traffic oscillation is presented by the discrete Fourier transform spectra (DFT spectra) under different frequencies, which is obtained by performing standard discrete Fourier transform (DFT) for the detrended traffic speed (Li et al., 2010). The magnitude of DFT spectra at each frequency indicates the magnitude of the speed oscillation at such frequency. The experiments are run under three scenarios, in which the advance warning is set at the optimal location, $977 \mathrm{~m}$ (Scenario 1), 400m (Scenario 2), and 1600m (Scenario 3) upstream to the off-ramp, respectively. The average results of ten runs for Lane 2 (the subject lane) and Lane 1 (the target lane) are showed in Figure 10 and Figure 11, respectively. 


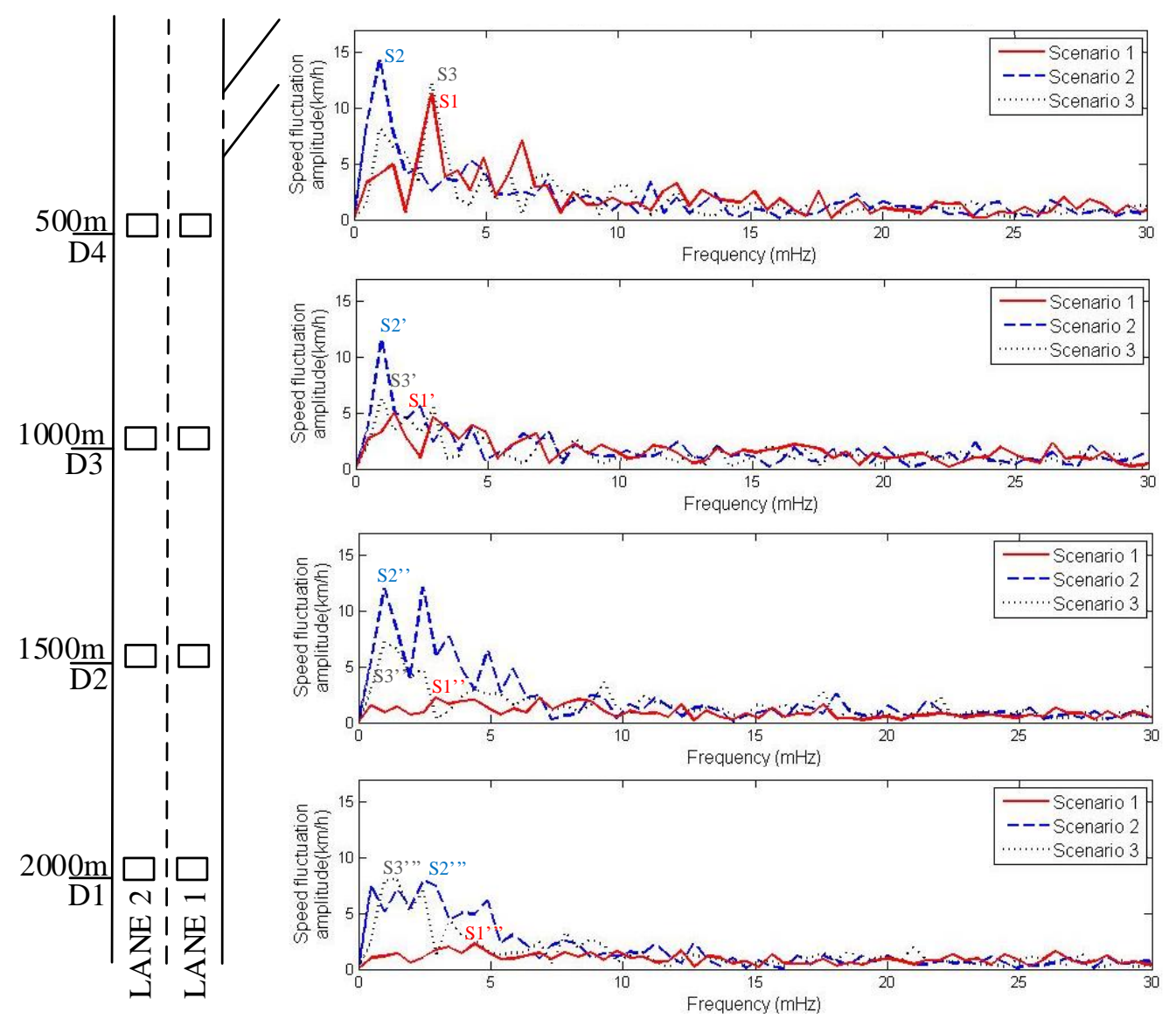

Figure 10. DFT spectra for detrended speed in Lane 2

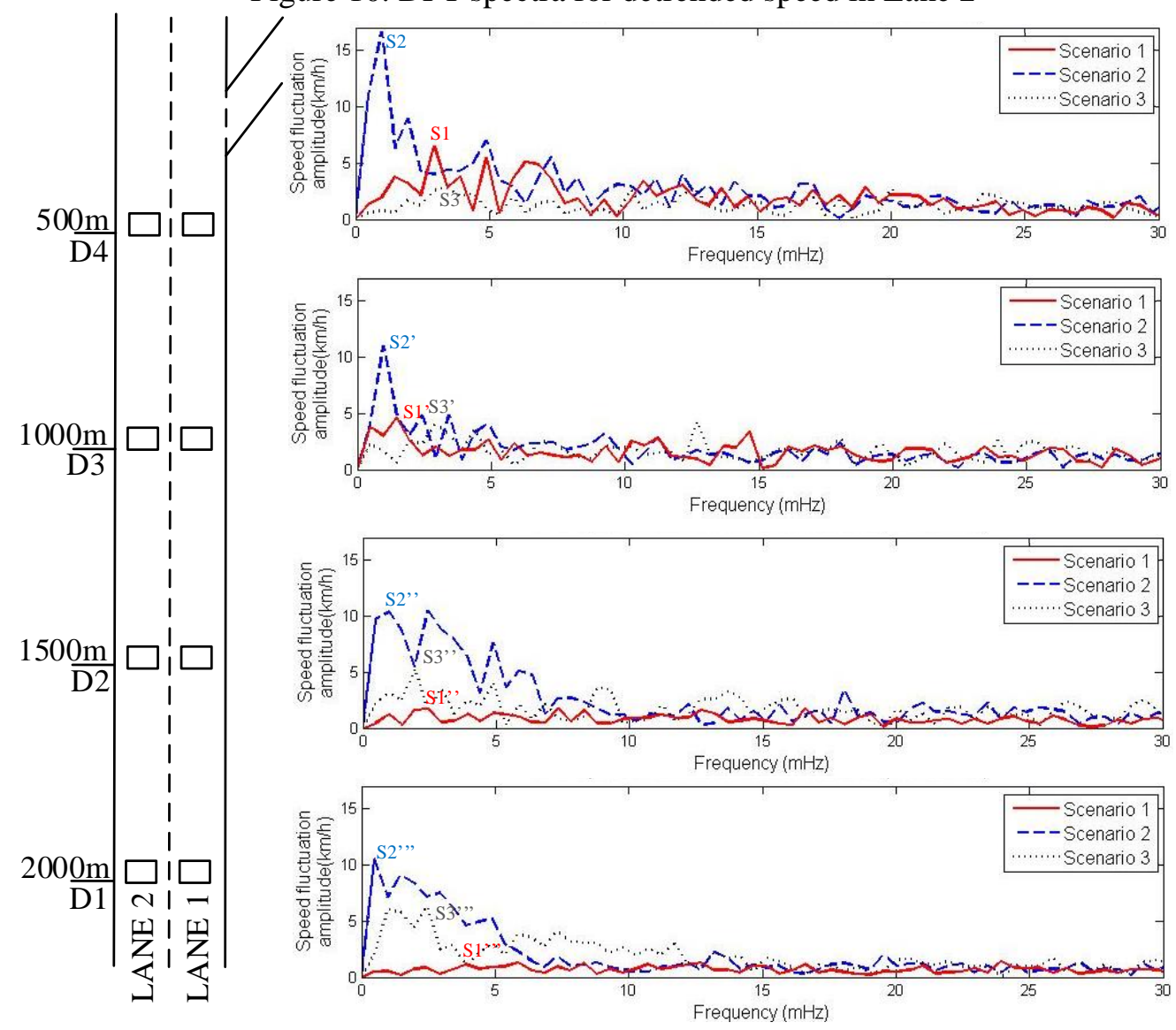

Figure 11. DFT spectra for detrended speed data in Lane 1 
The results in Figure 10 and Figure 11 indicate that placing the advance warning at the optimal location significantly mitigates traffic oscillation at different locations on both subject lane and target lane, even though traffic oscillation is not explicitly considered in the proposed optimization model. Specifically, Figure 10 indicates that the magnitude of speed fluctuation in Scenario 1 (such as the peak value $S 1=11.4 \mathrm{~km} / \mathrm{h}$ at D4) is lower than in Scenario 2 (such as the peak value $\mathrm{S} 2=14.5 \mathrm{~km} / \mathrm{h}$ at D4) and Scenario 3 (such as the peak value S3= $12.1 \mathrm{~km} / \mathrm{h}$ at D4) at locations D1-D4 under most of frequencies, even though at very few speed variation frequencies, the magnitude of fluctuation of Scenario 1 is slightly higher than Scenario 3 at locations D3 and D4. The similar observations are shown in Figure 11 too. Therefore, placing advance warning at the optimal location will mitigate traffic oscillation as well.

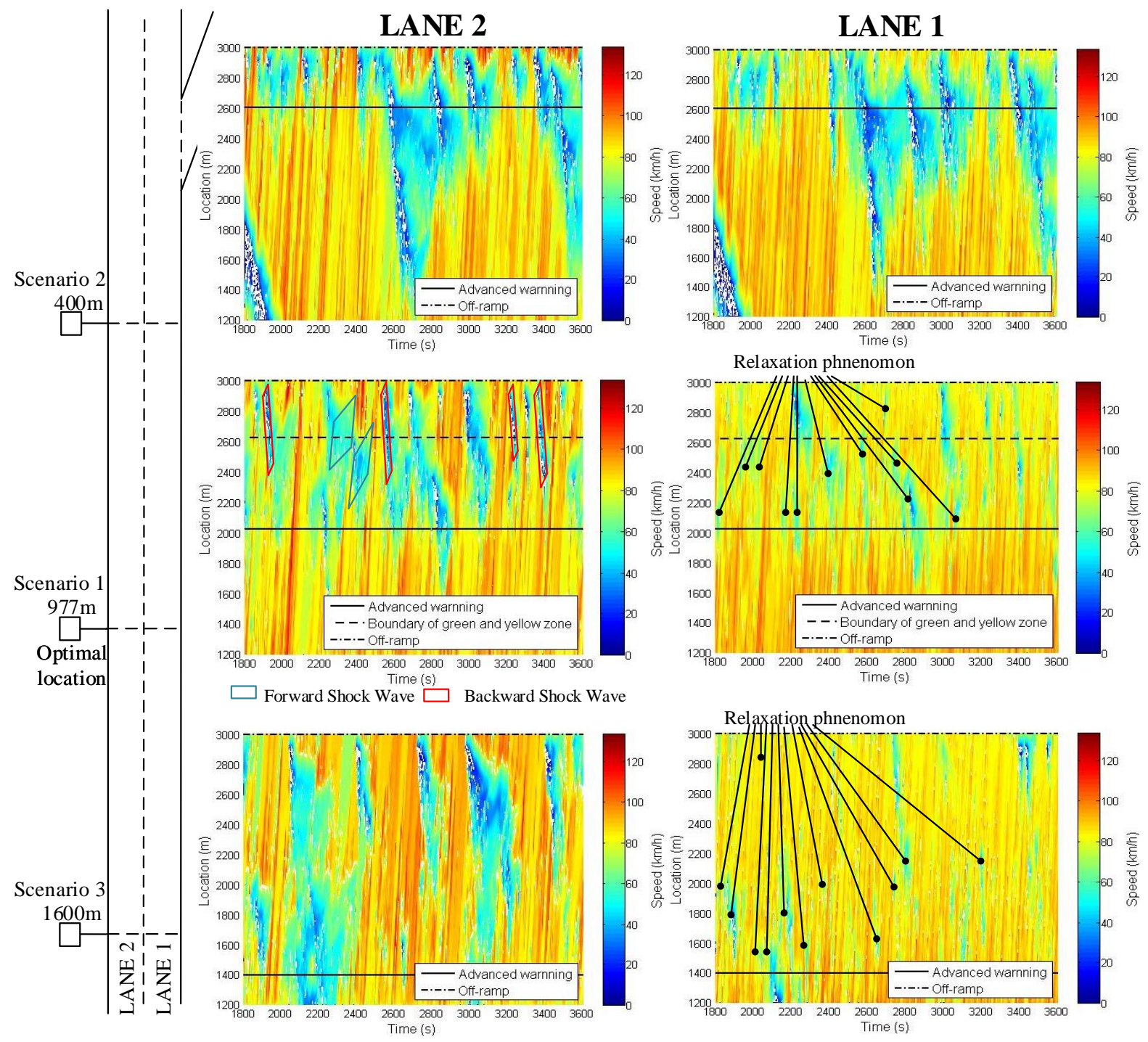

Figure 12. Speed-location-time diagram under different scenarios

This study last discusses the observations on the shock waves, gradual traffic state change and relaxation phenomenon shown in our experiments, according to the speed-location-time diagram shown in Figure 12, which is based on the trajectories data in the experiments to detect traffic oscillation. The solid dark line (the dash dot line) in each figure represents the location of the advancing warning (the off-ramp). The dash line in each figure of Scenario 1 represents the boundary of the green and yellow zones. Mainly, we present the following two key observations, which support some of our assumptions on lane change impact modeling.

First of all, the results in Scenario 1 indicate that D-MLC maneuvers which cause both forward and backward shock waves (see the dark blue areas in the figures), mainly occur in the yellow zone of Lane 2, while S-MLC maneuvers which cause gradual traffic slowing down (see the areas whose color 
changes from yellow to orange in the figures) mainly occur in the green zone of Lane 2. Moreover, it is observed that as the advance warning is moving away from the off-ramp (from $400 \mathrm{~m}$ in Scenario 2 to $1600 \mathrm{~m}$ in Scenario 3), severe shock waves on Lane 2 become less and less. This indicates the shrinking of the yellow zone and the expanding of the green zone in the area downstream of the advance warning on Lane 2. All these observations are consistent to our lane change impact analysis and modeling developed in Sections 3.2 and 3.4 for the green and yellow zones on Lane 2. At the same time, it is observed that both the green and yellow zones on Lane 1 in Scenarios 1 to 3 do not have significant shock waves. Instead, the traffic experiences a gradual evolution (the traffic gradually slowing down in the area from the advanced warning to the off-ramp; the corresponding color in this area changes from orange to yellow in the figures), which is consistent to our analysis for Lane 1 in Section 3.2.

Second, we provide the discussions on the observed relaxation phenomenon illustrated in Scenarios 1 to 3 in Figure 12, in which the lane changer or/and its following vehicle slightly decelerated to "relax" to a more comfortable spacing. First of all, it was noticed that the relaxation phenomenon scattered in the whole area downstream of the advance warning with similar amount (51 and 46 times of the relaxation phenomenon in Scenario 1 and Scenario 3 respectively) and they triggered similar deceleration processes. This observations suggested that the occurrence and the effect of the relaxation phenomenon are not closely related to the locations of lane change maneuvers. Moreover, based on our experiment setting, there are about 195 vehicles will conduct MLC from Lane 2 to the off-ramp. Thus, we observed around $25 \%$ of lane change involving the relaxation phenomenon in Scenario 1 and Scenario 3. It again confirmed the finding given in Leclercq et al. (2007) that the relaxation phenomenon will not happen very frequently in off-ramp condition. These observations support our discussion on ignoring the relaxation phenomenon in the proposed study.

\section{Conclusion}

Currently, when a driver performs a lane change nearby an off-ramp, he/she may start his/her lane change intention based on the advance warning which is located upstream to the off-ramp according engineering experiences, or fully depending on drives' previous driving experiences. However, due to traffic dynamics, neither of them work efficiently to avoid improper/rush lane change behaviors. Consequently, they frequently occur in the vicinity of the off-ramp and jeopardize traffic safety and efficacy. Even though this issue around work zones obtained more attentions in literature, the state of the art shows that we are lack of rigorous methods to find the optimal location of the advancing warning to efficiently guide the mandatory lane change maneuvers at off-ramp, lane division or lane merge/closure. This view motivates the proposed research.

This study seeks to develop the mathematical approaches, which identify the optimal location to set an advance warning to protect enough safe and smooth lane change opportunities as well as to sustain traffic efficiency in the vicinity of a highway off-ramp. The proposed approaches consider that the area downstream of the advance warning is consisted of two zones: the green and yellow zones corresponding to their respective most likely lane change maneuvers: S-MLC and D-MLC. Thus, to find the optimal location of the advance warning is equally to identify the location and length of the corresponding green and yellow zones. An optimization model is established to search the optimal green and yellow zones with the objective to minimize the time delay resulting from both S-MLC and DMLC maneuvers, subject to the constraints related to safe lane change opportunities and road length limits. The main challenges introduced in the optimization model are capturing lane change opportunity and the time delay resulting from S-MLC and D-MLC maneuvers. This study develops the probabilistic method combined with the gap acceptance model to address the former challenge. And then, this study analyzes the traffic state evaluation process resulting from the S-MLC maneuvers. Through combining the traffic state evolution with macroscopic traffic flow models, this study develops the closed-form formulations to capture the variation of traffic state (flow rate, density and volume) in spatial dimension. Furthermore, shock wave analysis is adopted to analyze the time delay caused by D-MLC maneuvers in the yellow zone. The developed optimization model is specified by the above formulations and solved by the grid search algorithm. Numerical experiments are conducted based on the simulation model in Paramics 6.9.3 to validate the efficiency of the proposed approaches. The experiment results indicate that our approaches work efficiently to identify the optimal location to set the advancing warning for the highway off-ramp so that the traffic delay resulting from MLC is minimized. The corresponding green and yellow zones obtained from the OPT model are consistent with the simulation results very 
well under different traffic regimes. Moreover, our experiments indicate that placing the advance warning at the optimal location will efficiently mitigate the capacity drop and traffic oscillation as well.

Several promising future research is stemmed from this study. First, the proposed methodology can be transferred to the cases of lane merge/closure at work zones beyond the scenario of the off-ramp studied in this paper. The current formulations to capture the time delay resulting from S-MLC and DMLC need to be revisited. Second, the proposed methodology can be further developed to address the mandatory lane change on a road segment with more than two lanes. The immediate challenge is to capture the traffic state evolution of the lane in the middle position, which includes both switch-in flow and switch-out flow. In addition, the analyses of the shock wave may be more complicated. Third, the present study only counts the traffic variation over the spatial dimension. A promising future work is to integrate spatial-temporal traffic flow dynamics, which is expected to give more efficient lane change guidance in real-time, but leads to the challenges in the formulation development and stability. This research team interests in working on them in near future.

\section{Acknowledgments}

This research is partially supported by the National Science Foundation Award: \# 1436786. The authors are grateful to the reviewers' efforts and their valuable comments.

\section{Reference}

Ahmed, K. I., 1999. Modeling Drivers' Acceleration and Lane-changing Behavior, PhD Dissertation, Department of Civil and Environmental Engineering, MIT.

Ahn, S., Cassidy, M. J., 2007. Freeway traffic oscillations and vehicle lane-change maneuvers. Proc., 17Th International Symposium of Transportation and Traffic Theory, 691-710, Amsterdam, Elsevier

Ahn, S., Laval, J., Cassidy, M.J., 2010. Merging and diverging effects on freeway traffic oscillations: theory and observation. Transportation Research Record: Journal of the Transportation Research Board 2188, 1-8.

Ardelt, M., Coester, C., Kaempchen, N., 2012. Highly automated driving on freeways in real traffic using a probabilistic framework. Intelligent Transportation Systems, IEEE Transactions on, 13(4), 1576-1585.

Cassidy, M.J., Anani, S.B., Haigwood, J.M., 2002. Study of freeway traffic near an off-ramp. Transportation Research Part A 36 (6), 563-572

Choudhury, C. F., 2002. Modeling Lane-changing Behavior in Presence of Exclusive Lanes. Master degree thesis. Department of Civil and Environmental Engineering, MIT

Daganzo, C.F., 1999. Remarks on traffic flow modeling and its applications. In: Brilon, W., Huber, F., Schreckenberg, M., Wallentowitz, H. (Eds.), Proceedings of Traffic and Mobility: Simulation, Economics and Environment. Springer Verlag, pp. 105-115

Daganzo, C. F., 2002a. A behavioral theory of multi-lane traffic flow. Part I: Long homogeneous freeway sections. Transportation Research Part B: Methodological, 36(2), 131-158.

Daganzo, C. F., 2002b. A behavioral theory of multi-lane traffic flow. Part II: Merges and the onset of congestion. Transportation Research Part B: Methodological, 36(2), 159-169.

FHWA., 1998. CORSIM User Mannual (1.04 ed). US DOT, Office of Safety and Traffic Operation R\&D, Intelligent Systems and Technology Division (HSR-10), McLean, Virginia.

FHWA, 2009. Manual on uniform traffic control devices (2009 ed.). Washington, D.C.: U.S. Dept. of Transportation.

Gurupackiam, S., Jones Jr, S. L., Turner, D. S., 2010. Characterization of Arterial Traffic Congestion Through Analysis of Operational Parameters (Gap Acceptance and Lane Changing), UTCA Report Number 07112.

Halati, A., Lieu, H., Walker, S., 1997. CORSIM - Corridor Traffic Simulation Model, in Proceedings of the Traffic Congestion and Traffic Safety in the 21st Century Conference, pp. 570-576.

Hou, Y., Edara, P., Sun, C., 2012. A Genetic Fuzzy System for Modeling Mandatory Lane Changing. 2012 15th International IEEE Conference on Intelligent Transportation Systems. Anchorage, Alaska, USA, September 16-19, 2012

Jin, W. L., Zhang, Y., 2005. Paramics simulation of periodic oscillations caused by network geometry. Transportation Research Record: Journal of the Transportation Research Board 1934, 188-196. 
Jin, W. L., 2009. Asymptotic traffic dynamics arising in diverge-merge networks with two intermediate links. Transportation Research Part B 43 (5), 575-595.

Jin, W. L. 2010. A kinematic wave theory of lane-changing traffic flow. Transportation research part B: methodological, 44(8), 1001-1021.

Jin, W. L. 2013. A multi-commodity Lighthill-Whitham-Richards model of lane-changing traffic flow. Transportation Research Part B: Methodological, 57, 361-377.

Jula, H., Kosmatopoulos, E. B., Ioannou, P., 2000. Collision avoidance analysis for lane changing and merging. Vehicular Technology, IEEE Transactions on, 49(6), 2295-2308.

Laval, J. A., Daganzo, C. F., 2006. Lane-changing in traffic streams. Transportation Research Part B: Methodological, 40(3), 251-264.

Laval, J. A., Leclercq, L. 2008. Microscopic modeling of the relaxation phenomenon using a macroscopic lane-changing model. Transportation Research Part B: Methodological, 42(6), 511-522.

Leclercq, L., Chiabaut, N., Laval, J., Buisson, C. 2007. Relaxation phenomenon after lane changing: experimental validation with NGSIM data set. Transportation Research Record: Journal of the Transportation Research Board, 1999, 79-85.

Li, X., Peng, F., Ouyang, Y. 2010. Measurement and estimation of traffic oscillation properties. Transportation Research Part B: Methodological, 44(1), 1-14.

Li, X., Ouyang, Y., 2011. Characterization of traffic oscillation propagation under nonlinear carfollowing laws. Transportation research part B: methodological, 45(9), 1346-1361.

HCM 2010: Highway Capacity Manual. Washington, D.C.: Transportation Research Board, 2010.

Mauch, M., Cassidy, M. J., 2002. Freeway traffic oscillations: observations and predictions. In: Taylor, M.A.P. (Ed.), 15th International Symposium on Transportation and Traffic Theory. PergamoonElsevier, Oxford, UK, pp. 653-674.

McCoy, P., Pesti, G., 2001. Dynamic late merge-control concept for work zones on rural interstate highways. Transportation Research Record: Journal of the Transportation Research Board, (1745), 20-26

Michigan DOT, 2004. Development and Evaluation of an advanced dynamic lane merge traffic control system for 3 to 2 lane transition areas in work zones (No. Research Report RC-1451).

Munoz, J. C., Daganzo, C. F. 2002. The bottleneck mechanism of a freeway diverge. Transportation Research Part A: Policy and Practice, 36(6), 483-50

Park, H., Smith, B. L., 2012. Investigating benefits of intellidrive in freeway operations: Lane changing advisory case study. Journal of Transportation Engineering, 138(9), 1113-1122.

Radwan, E., Zaidi, Z., Harb, R., 2011. Operational Evaluation of Dynamic Lane Merging In Work Zones with Variable Speed Limits. Procedia-Social and Behavioral Sciences, 16, 460-469.

Schofield, K., 2005. U.S. Patent No. 6,882,287. Washington, DC: U.S. Patent and Trademark Office.

Talmadge, S., Chu, R., Eberhard, C., Jordan, K., Moffa, P., 2000. Development of performance specifications for collisions avoidance systems for lane change crashes (No. HS-809 414,).

Tarko, A. P., Shamo, D., Wasson, J., 1999. Indiana Lane Merge System for Work Zones on Rural Freeways. Journal of Transportation Engineering, Vol. 125, No. 5, pp.415-420

Toledo, T., Koutsopoulos, H., Ben-Akiva, M. 2003. Modeling integrated lane-changing behavior. Transportation Research Record: Journal of the Transportation Research Board, (1857), 30-38.

Toledo, T., Koutsopoulos, H., Ben-Akiva, M., 2007. Integrated driving behavior modeling. Transportation Research Part C: Emerging Technologies, 15(2), 96-112.

Gerlough, D. L., Huber, M. J. 1975. Traffic Flow Theory - A Monograph. Transportation Research Board, Special Report 165, Washington D. C.

Vahidi, A., Eskandarian, A., 2003. Research advances in intelligent collision avoidance and adaptive cruise control. Intelligent Transportation Systems, IEEE Transactions on, 4(3), 143-153.

Zheng, Z., Ahn, S., Chen, D., Laval, J., 2013. The effects of lane-changing on the immediate follower: Anticipation, relaxation, and change in driver characteristics. Transportation research part C: emerging technologies, 26, 367-379.

Zheng, Z., 2014. Recent developments and research needs in modeling lane changing. Transportation research part B: methodological, 60, 16-32. 


\section{Appendix A}

The closed-form formulations for the time delay in the green zone will be given below. All the integration formulations are solved by Wolfram Mathematica 9.0.

\section{(1). Time delay $t_{1 \rightarrow 1}^{g}$ under Free flow condition}

$$
\begin{aligned}
t_{1 \rightarrow 1}^{g}=\int_{0}^{L_{g}}\left(\frac{d x}{v_{1}^{g}(\mathrm{x})}\right. & \left.-\frac{d x}{v_{1}^{g}(0)}\right) \\
& =\left\{2 \operatorname{ArcTan}\left(\frac{\sqrt{\mathrm{c}_{1}-4 F_{g}\left(L_{g}\right) \alpha q_{2}^{i n}}}{\mathrm{c}_{2}}\right) \sqrt{k_{j} \mathrm{c}_{2}}\right. \\
& \left.-\left[2 \operatorname{ArcTanh}\left[\sqrt{\frac{\mathrm{c}_{1}-4 F_{g}\left(L_{g}\right) \alpha q_{2}^{i n}}{k_{j} v_{f}}}\right]-L_{g} \mathrm{c}_{3}+\log \left[q_{1}^{i n}+F_{a}\left(L_{a}\right) \alpha q_{2}^{i n}\right]\right] k_{j} \sqrt{v_{f}}\right\} \\
& /\left(2 \mathrm{c}_{3} \sqrt{v_{f}}\left(q_{1}^{i n}+\alpha q_{2}^{i n}\right)\right) \\
& -\left\{2 \operatorname{ArcTan}\left(\frac{\sqrt{\mathrm{c}_{1}-4 p \alpha q_{2}^{i n}}}{\mathrm{c}_{2}}\right) \sqrt{k_{j} \mathrm{c}_{2}}\right. \\
& \left.-\left[2 \operatorname{ArcTanh}\left[\sqrt{\frac{\mathrm{c}_{1}-4 p \alpha q_{2}^{i n}}{k_{j} v_{f}}}\right]+\log \left[q_{1}^{i n}+p \alpha q_{2}^{i n}\right]\right] k_{j} \sqrt{v_{f}}\right\} /\left(2 \mathrm{c}_{3} \sqrt{v_{f}}\left(q_{1}^{i n}+\alpha q_{2}^{i n}\right)\right) \\
& -\frac{L_{g}}{v_{1}^{g}(0)}
\end{aligned}
$$

Where:

$$
\begin{gathered}
\mathrm{c}_{1}=-4 q_{1}^{i n}+k_{j} v_{f} \\
\mathrm{c}_{2}=\sqrt{-4 q_{1}^{i n}+k_{j} v_{f}-4 \alpha q_{2}^{i n}} \\
\mathrm{c}_{3}=\frac{\left|v_{2}^{g}-v_{1}^{g}\right| k_{1}^{g} \log [1-p]}{v_{2}^{g}}
\end{gathered}
$$

(2). Time delay $t_{1 \rightarrow 1}^{g}$ under congestion flow condition

$$
\begin{aligned}
t_{1 \rightarrow 1}^{g}=\int_{0}^{L_{g}}\left(\frac{d x}{v_{1}^{g}(\mathrm{x})}\right. & \left.-\frac{d x}{v_{1}^{g}(0)}\right) \\
& =\left\{2 \operatorname{ArcTan}\left(\frac{\sqrt{\mathrm{c}_{1}-4 F_{g}\left(L_{g}\right) \alpha q_{2}^{i n}}}{\mathrm{c}_{2}}\right) \sqrt{k_{j} \mathrm{c}_{2}}\right. \\
& \left.-\left[2 \operatorname{ArcTanh}\left[\sqrt{\frac{\mathrm{c}_{1}-4 F_{g}\left(L_{g}\right) \alpha q_{2}^{i n}}{k_{j} v_{f}}}\right]+L_{g} \mathrm{c}_{3}-\log \left[q_{1}^{i n}+F_{g}\left(L_{g}\right) \alpha q_{2}^{i n}\right]\right] k_{j} \sqrt{v_{f}}\right\} \\
& /\left(2 \mathrm{c}_{3} \sqrt{v_{f}}\left(q_{1}^{i n}+\alpha q_{2}^{i n}\right)\right) \\
& -\left\{2 \operatorname{ArcTan}\left(\frac{\sqrt{\mathrm{c}_{1}-4 p \alpha q_{2}^{i n}}}{\mathrm{c}_{2}}\right) \sqrt{k_{j} \mathrm{c}_{2}}\right. \\
& \left.-\left[2 \operatorname{ArcTanh}\left[\sqrt{\frac{\mathrm{c}_{1}-4 p \alpha q_{2}^{i n}}{k_{j} v_{f}}}\right]-\log \left[q_{1}^{i n}+p \alpha q_{2}^{i n}\right]\right] k_{j} \sqrt{v_{f}}\right\} /\left(2 \mathrm{c}_{3} \sqrt{v_{f}}\left(q_{1}^{i n}+\alpha q_{2}^{i n}\right)\right) \\
& -\frac{L_{g}}{v_{1}^{g}(0)}
\end{aligned}
$$


(3). Time delay $t_{2 \rightarrow 2}^{g}$ under free flow condition

$$
\begin{aligned}
t_{2 \rightarrow 2}^{g}=\int_{0}^{L_{g}}\left(\frac{d x}{v_{2}^{g}(x)}\right. & \left.-\frac{d x}{v_{2}^{g}(0)}\right)= \\
& =\left\{2 \operatorname{ArcTan}\left(\frac{\sqrt{\mathrm{c}_{4}+4 F_{g}\left(L_{g}\right) \alpha q_{2}^{i n}}}{\mathrm{c}_{5}}\right) \sqrt{k_{j} \mathrm{c}_{5}}\right. \\
& \left.-\left[2 \operatorname{ArcTanh}\left[\sqrt{\frac{\mathrm{c}_{4}+4 F_{g}\left(L_{g}\right) \alpha q_{2}^{i n}}{k_{j} v_{f}}}\right]-L_{g} \mathrm{c}_{3}+\log \left[q_{2}^{i n}-F_{g}\left(L_{g}\right) \alpha q_{2}^{i n}\right]\right] k_{j} \sqrt{v_{f}}\right\} \\
& /\left(2 c_{3} \sqrt{v_{f}}\left(q_{2}^{i n}-\alpha q_{2}^{i n}\right)\right) \\
& -\left\{2 \operatorname{ArcTan}\left(\frac{\sqrt{\mathrm{c}_{4}+4 p \alpha q_{2}^{i n}}}{\mathrm{c}_{5}}\right) \sqrt{k_{j}} \mathrm{c}_{5}\right. \\
& \left.-\left[2 \operatorname{ArcTanh}\left[\sqrt{\frac{\mathrm{c}_{4}+4 p \alpha q_{2}^{i n}}{k_{j} v_{f}}}\right]+\log \left[q_{2}^{i n}-p \alpha q_{2}^{i n}\right]\right] k_{j} \sqrt{v_{f}}\right\} /\left(2 c_{3} \sqrt{v_{f}}\left(q_{2}^{i n}-\alpha q_{2}^{i n}\right)\right) \\
& -\frac{L_{g}}{v_{2}^{g}(0)}
\end{aligned}
$$

Where:

$$
\begin{gathered}
\mathrm{c}_{4}=-4 q_{2}^{i n}+k_{j} v_{f} \\
\mathrm{c}_{5}=\sqrt{--4 q_{2}^{i n}+k_{j} v_{f}+4 \alpha q_{2}^{i n}}
\end{gathered}
$$

(4). Time delay $t_{2 \rightarrow 2}^{g}$ under congestion flow condition

$$
\begin{aligned}
t_{2 \rightarrow 2}^{g}=\int_{0}^{L_{g}}\left(\frac{d x}{v_{2}^{g}(x)}\right. & \left.-\frac{d x}{v_{2}^{g}(0)}\right)= \\
= & \left\{2 \operatorname{ArcTan}\left(\frac{\sqrt{c_{4}+4 F_{g}\left(L_{g}\right) \alpha q_{2}^{i n}}}{c_{5}}\right) \sqrt{k_{j} c_{5}}\right. \\
& \left.-\left[2 \operatorname{ArcTanh}\left[\sqrt{\frac{c_{4}+4 F_{g}\left(L_{g}\right) \alpha q_{2}^{i n}}{k_{j} v_{f}}}\right]+L_{a} c_{3}-\log \left[q_{2}^{i n}-F_{g}\left(L_{g}\right) \alpha q_{2}^{i n}\right]\right] k_{j} \sqrt{v_{f}}\right\} \\
& /\left(2 c_{3} \sqrt{v_{f}}\left(q_{2}^{i n}-\alpha q_{2}^{i n}\right)\right) \\
& -\left\{2 \operatorname{ArcTan}\left(\frac{\sqrt{c_{4}+4 p \alpha q_{2}^{i n}}}{c_{5}}\right) \sqrt{k_{j}} c_{5}\right. \\
& \left.-\left[2 \operatorname{ArcTanh}\left[\sqrt{\frac{c_{4}+4 p \alpha q_{2}^{i n}}{k_{j} v_{f}}}\right]-\log \left[q_{2}^{i n}-p \alpha q_{2}^{i n}\right]\right] k_{j} \sqrt{v_{f}}\right\} /\left(2 c_{3} \sqrt{v_{f}}\left(q_{2}^{i n}-\alpha q_{2}^{i n}\right)\right) \\
& -\frac{L_{g}}{v_{2}^{a}(0)}
\end{aligned}
$$




\section{(5). Time delay $t_{2 \rightarrow 1}^{g}$ when both Lane 1 and Lane 2 are under free flow condition}

$$
\begin{aligned}
& t_{2 \rightarrow 1}^{g}=E\left(T_{2 \rightarrow 1}^{g}\right)=\int_{0}^{L_{g}} T_{2 \rightarrow 1}^{g} P_{g}(r) d r=\int_{0}^{L_{g}} T_{2 \rightarrow 1}^{g} d F_{g}(r)=\left.T_{2 \rightarrow 1}^{g} F_{g}(r)\right|_{0} ^{L_{g}}-\int_{0}^{L_{g}} F_{g}(r) d T_{2 \rightarrow 1}^{g} \\
& =\left.T_{2 \rightarrow 1}^{g} F_{g}(r)\right|_{0} ^{L_{g}}-\int_{0}^{L_{g}} F_{g}(r)\left(\frac{1}{v_{2}^{g}(r)}-\frac{1}{v_{1}^{g}(r)}\right) d r \\
& =t_{2 \rightarrow 2}^{g} * F_{g}\left(L_{g}\right)-t_{1 \rightarrow 1}^{g} * \mathrm{p}-\frac{\log \left[q_{2}^{i n}-\alpha F_{g}\left(L_{g}\right) q_{2}^{i n}\right] k_{j}}{2 c_{3}(1-\alpha) \alpha q_{2}^{i n}}-\frac{\operatorname{ArcTanh}\left[\frac{\sqrt{c_{1}-4 \alpha F_{g}\left(L_{g}\right) q_{2}^{i n}}}{c_{2}}\right] \sqrt{k_{j}} c_{2}}{c_{3} \sqrt{v_{f}}\left(q_{1}^{i n}+\alpha q_{2}^{i n}\right)} \\
& -\frac{\log \left[q_{1}^{i n}+\alpha F_{g}\left(L_{g}\right) q_{2}^{i n}\right] k_{j} q_{1}^{i n}}{2 c_{3}\left(q_{1}^{i n}+\alpha q_{2}^{i n}\right) \alpha q_{2}^{i n}}+\frac{L_{g} k_{j}\left(q_{1}^{i n}-q_{2}^{i n}+2 \alpha q_{2}^{i n}\right)}{2\left(q_{2}^{i n}-\alpha q_{2}^{i n}\right)\left(q_{1}^{i n}+\alpha q_{2}^{i n}\right)}+\frac{\operatorname{ArcTanh}\left[\frac{\sqrt{c_{4}+4 \alpha F_{g}\left(L_{g}\right) q_{2}^{i n}}}{c_{5}}\right] \sqrt{k_{j}} c_{5}}{c_{3} \sqrt{v_{f}}\left(q_{2}^{i n}-\alpha q_{2}^{i n}\right)} \\
& -\frac{\operatorname{ArcTanh}\left[\sqrt{\frac{c_{4}+4 \alpha F_{g}\left(L_{g}\right) q_{2}^{i n}}{k_{j} v_{f}}}\right] k_{j} \quad \operatorname{ArcTanh}\left[\sqrt{\frac{c_{1}-4 \alpha F_{g}\left(L_{g}\right) q_{2}^{i n}}{k_{j} v_{f}}}\right] k_{j} q_{1}^{i n}}{c_{3}\left(q_{2}^{i n}-\alpha q_{2}^{i n}\right)(-\alpha)}-\frac{k_{j} \sqrt{\frac{c_{1}-4 \alpha F_{g}\left(L_{g}\right) q_{2}^{i n}}{k_{j} v_{f}}}}{c_{3} k_{j} v_{f}\left(q_{1}^{i n}+\alpha q_{2}^{i n}\right) q_{2}^{i n}} \\
& +\frac{k_{j} \sqrt{\frac{c_{4}+4 \alpha F_{g}\left(L_{g}\right) q_{2}^{i n}}{k_{j} v_{f}}}}{c_{3} \alpha q_{2}^{i n}}+\frac{\log \left[q_{2}^{i n}-p \alpha q_{2}^{i n}\right] k_{j} q_{2}^{i n}}{2 c_{3}\left(q_{2}^{i n}-\alpha q_{2}^{i n}\right) \alpha q_{2}^{i n}}+\frac{\operatorname{ArcTanh}\left[\frac{\sqrt{c_{1}-4 p \alpha q_{2}^{i n}}}{c_{2}}\right] \sqrt{k_{j}} c_{2}}{c_{3} \sqrt{v_{f}}\left(q_{1}^{i n}+\alpha q_{2}^{i n}\right)} \\
& +\frac{\log \left[q_{1}^{i n}+p \alpha q_{2}^{i n}\right] k_{j} q_{1}^{i n}}{2 c_{3} \alpha q_{2}^{i n}\left(q_{1}^{i n}+\alpha q_{2}^{i n}\right)}-\frac{\operatorname{ArcTanh}\left[\frac{\sqrt{c_{4}+4 p \alpha q_{2}^{i n}}}{c_{5}}\right] \sqrt{k_{j}} c_{5} \quad \operatorname{ArcTanh}\left[\sqrt{\frac{c_{4}+4 p \alpha q_{2}^{i n}}{k_{j} v_{f}}}\right] k_{j}}{c_{3} \sqrt{v_{f}}\left(q_{2}^{i n}-\alpha q_{2}^{i n}\right)}+\frac{c_{3}\left(q_{2}^{i n}-\alpha q_{2}^{i n}\right)(-\alpha)}{c^{i n}} \\
& +\frac{\operatorname{ArcTanh}\left[\sqrt{\frac{c_{1}-4 p \alpha q_{2}^{i n}}{k_{j} v_{f}}}\right] k_{j} q_{1}^{i n}}{c_{3}\left(q_{1}^{i n}+\alpha q_{2}^{i n}\right) \alpha q_{2}^{i n}}-\frac{k_{j} \sqrt{\frac{c_{1}-4 p \alpha q_{2}^{i n}}{k_{j} v_{f}}}}{c_{3} \alpha q_{2}^{i n}}-\frac{k_{j} \sqrt{\frac{c_{4}+4 p \alpha q_{2}^{i n}}{k_{j} v_{f}}}}{c_{3} \alpha q_{2}^{i n}}
\end{aligned}
$$


(6). Time delay $t_{2 \rightarrow 1}^{g}$ when both Lane 1 and Lane 2 are under congestion flow

$$
\begin{aligned}
& t_{2 \rightarrow 1}^{g}=E\left(T_{2 \rightarrow 1}^{g}\right)=\int_{0}^{L_{g}} T_{2 \rightarrow 1}^{g} P_{g}(r) d r=\int_{0}^{L_{g}} T_{2 \rightarrow 1}^{g} d F_{g}(r)=\left.T_{2 \rightarrow 1}^{g} F_{g}(r)\right|_{0} ^{L_{g}}-\int_{0}^{L_{g}} F_{g}(r) d T_{2 \rightarrow 1}^{g} \\
& =\left.T_{2 \rightarrow 1}^{g} F_{g}(r)\right|_{0} ^{L_{g}}-\int_{0}^{L_{g}} F_{g}(r)\left(\frac{1}{v_{2}^{g}(r)}-\frac{1}{v_{1}^{g}(r)}\right) d r \\
& =t_{2 \rightarrow 2}^{g} * F_{g}\left(L_{g}\right)-t_{1 \rightarrow 1}^{g} * \mathrm{p}-\frac{\log \left[q_{2}^{i n}-\alpha F_{g}\left(L_{g}\right) q_{2}^{i n}\right] k_{j}}{2 c_{3}(1-\alpha) \alpha q_{2}^{i n}}+\frac{\operatorname{ArcTanh}\left[\frac{\sqrt{c_{1}-4 \alpha F_{g}\left(L_{g}\right) q_{2}^{i n}}}{c_{2}}\right] \sqrt{k_{j}} c_{2}}{c_{3} \sqrt{v_{f}}\left(q_{1}^{i n}+\alpha q_{2}^{i n}\right)} \\
& -\frac{\log \left[q_{1}^{i n}+\alpha F_{g}\left(L_{g}\right) q_{2}^{i n}\right] k_{j} q_{1}^{i n}}{2 c_{3}\left(q_{1}^{i n}+\alpha q_{2}^{i n}\right) \alpha q_{2}^{i n}}+\frac{L_{g} k_{j}\left(q_{1}^{i n}-q_{2}^{i n}+2 \alpha q_{2}^{i n}\right)}{2\left(q_{2}^{i n}-\alpha q_{2}^{i n}\right)\left(q_{1}^{i n}+\alpha q_{2}^{i n}\right)}-\frac{\operatorname{ArcTanh}\left[\frac{\sqrt{c_{4}+4 \alpha F_{g}\left(L_{g}\right) q_{2}^{i n}}}{c_{5}}\right] \sqrt{k_{j}} c_{5}}{c_{3} \sqrt{v_{f}}\left(q_{2}^{i n}-\alpha q_{2}^{i n}\right)} \\
& +\frac{\operatorname{ArcTanh}\left[\sqrt{\frac{c_{4}+4 \alpha F_{g}\left(L_{g}\right) q_{2}^{i n}}{k_{j} v_{f}}}\right] k_{j} \quad \operatorname{ArcTanh}\left[\sqrt{\frac{c_{1}-4 \alpha F_{g}\left(L_{g}\right) q_{2}^{i n}}{k_{j} v_{f}}}\right] k_{j} q_{1}^{i n}}{c_{3}\left(q_{2}^{i n}-\alpha q_{2}^{i n}\right)(-\alpha)}+\frac{k_{j} \sqrt{\frac{c_{1}-4 \alpha F_{g}\left(L_{g}\right) q_{2}^{i n}}{k_{j} v_{f}}}}{c_{3} k_{j} v_{f}\left(q_{1}^{i n}+\alpha q_{2}^{i n}\right) q_{2}^{i n}} \\
& -\frac{k_{j} \sqrt{\frac{c_{4}+4 \alpha F_{g}\left(L_{g}\right) q_{2}^{i n}}{k_{j} v_{f}}}}{c_{3} \alpha q_{2}^{i n}}+\frac{\log \left[q_{2}^{i n}-p \alpha q_{2}^{i n}\right] k_{j} q_{2}^{i n}}{2 c_{3}\left(q_{2}^{i n}-\alpha q_{2}^{i n}\right) \alpha q_{2}^{i n}}-\frac{\operatorname{ArcTanh}\left[\frac{\sqrt{c_{1}-4 p \alpha q_{2}^{i n}}}{c_{2}}\right] \sqrt{k_{j}} c_{2}}{c_{3} \sqrt{v_{f}}\left(q_{1}^{i n}+\alpha q_{2}^{i n}\right)} \\
& +\frac{\log \left[q_{1}^{i n}+p \alpha q_{2}^{i n}\right] k_{j} q_{1}^{i n}}{2 c_{3} \alpha q_{2}^{i n}\left(q_{1}^{i n}+\alpha q_{2}^{i n}\right)}+\frac{\operatorname{ArcTanh}\left[\frac{\sqrt{c_{4}+4 p \alpha q_{2}^{i n}}}{c_{5}}\right] \sqrt{k_{j}} c_{5}}{c_{3} \sqrt{v_{f}}\left(q_{2}^{i n}-\alpha q_{2}^{i n}\right)}-\frac{\operatorname{ArcTanh}\left[\sqrt{\frac{c_{4}+4 p \alpha q_{2}^{i n}}{k_{j} v_{f}}}\right] k_{j}}{c_{3}\left(q_{2}^{i n}-\alpha q_{2}^{i n}\right)(-\alpha)} \\
& -\frac{\operatorname{ArcTanh}\left[\sqrt{\frac{c_{1}-4 p \alpha q_{2}^{i n}}{k_{j} v_{f}}}\right] k_{j} q_{1}^{i n}}{c_{3}\left(q_{1}^{i n}+\alpha q_{2}^{i n}\right) \alpha q_{2}^{i n}}+\frac{k_{j} \sqrt{\frac{c_{1}-4 p \alpha q_{2}^{i n}}{k_{j} v_{f}}}}{c_{3} \alpha q_{2}^{i n}}+\frac{k_{j} \sqrt{\frac{c_{4}+4 p \alpha q_{2}^{i n}}{k_{j} v_{f}}}}{c_{3} \alpha q_{2}^{i n}}
\end{aligned}
$$


(7). Time delay $t_{2 \rightarrow 1}^{g}$ when Lane 1 is under free flow and Lane 2 is under congestion flow:

$$
\begin{aligned}
& t_{2 \rightarrow 1}^{g}=E\left(T_{2 \rightarrow 1}^{g}\right)=\int_{0}^{L_{g}} T_{2 \rightarrow 1}^{g} P_{g}(r) d r=\int_{0}^{L_{g}} T_{2 \rightarrow 1}^{g} d F_{g}(r)=\left.T_{2 \rightarrow 1}^{g} F_{g}(r)\right|_{0} ^{L_{g}}-\int_{0}^{L_{g}} F_{g}(r) d T_{2 \rightarrow 1}^{g} \\
& =\left.T_{2 \rightarrow 1}^{g} F_{g}(r)\right|_{0} ^{L_{g}}-\int_{0}^{L_{g}} F_{g}(r)\left(\frac{1}{v_{2}^{g}(r)}-\frac{1}{v_{1}^{g}(r)}\right) d r \\
& =t_{2 \rightarrow 2}^{g} * F_{g}\left(L_{g}\right)-t_{1 \rightarrow 1}^{g} * \mathrm{p}-\frac{\log \left[q_{2}^{i n}-\alpha F_{g}\left(L_{g}\right) q_{2}^{i n}\right] k_{j}}{2 c_{3}(1-\alpha) \alpha q_{2}^{i n}}+\frac{\operatorname{ArcTanh}\left[\frac{\sqrt{c_{1}-4 \alpha F_{g}\left(L_{g}\right) q_{2}^{i n}}}{c_{2}}\right] \sqrt{k_{j}} c_{2}}{c_{3} \sqrt{v_{f}}\left(q_{1}^{i n}+\alpha q_{2}^{i n}\right)} \\
& -\frac{\log \left[q_{1}^{i n}+\alpha F_{g}\left(L_{g}\right) q_{2}^{i n}\right] k_{j} q_{1}^{i n}}{2 c_{3}\left(q_{1}^{i n}+\alpha q_{2}^{i n}\right) \alpha q_{2}^{i n}}+\frac{L_{g} k_{j}\left(q_{1}^{i n}-q_{2}^{i n}+2 \alpha q_{2}^{i n}\right)}{2\left(q_{2}^{i n}-\alpha q_{2}^{i n}\right)\left(q_{1}^{i n}+\alpha q_{2}^{i n}\right)}+\frac{\operatorname{ArcTanh}\left[\frac{\sqrt{c_{4}+4 \alpha F_{g}\left(L_{g}\right) q_{2}^{i n}}}{c_{5}}\right] \sqrt{k_{j}} c_{5}}{c_{3} \sqrt{v_{f}}\left(q_{2}^{i n}-\alpha q_{2}^{i n}\right)} \\
& -\frac{\operatorname{ArcTanh}\left[\sqrt{\frac{c_{4}+4 \alpha F_{g}\left(L_{g}\right) q_{2}^{i n}}{k_{j} v_{f}}}\right] k_{j} \quad \operatorname{ArcTanh}\left[\sqrt{\frac{c_{1}-4 \alpha F_{g}\left(L_{g}\right) q_{2}^{i n}}{k_{j} v_{f}}}\right] k_{j} q_{1}^{i n}}{c_{3}\left(q_{2}^{i n}-\alpha q_{2}^{i n}\right)(-\alpha)}+\frac{k_{j} \sqrt{\frac{c_{1}-4 \alpha F_{g}\left(L_{g}\right) q_{2}^{i n}}{k_{j} v_{f}}}}{c_{3} k_{j} v_{f}\left(q_{1}^{i n}+\alpha q_{2}^{i n}\right) q_{2}^{i n}} \\
& +\frac{k_{j} \sqrt{\frac{c_{4}+4 \alpha F_{g}\left(L_{g}\right) q_{2}^{i n}}{k_{j} v_{f}}}}{c_{3} \alpha q_{2}^{i n}}+\frac{\log \left[q_{2}^{i n}-p \alpha q_{2}^{i n}\right] k_{j} q_{2}^{i n}}{2 c_{3}\left(q_{2}^{i n}-\alpha q_{2}^{i n}\right) \alpha q_{2}^{i n}}-\frac{\operatorname{ArcTanh}\left[\frac{\sqrt{c_{1}-4 p \alpha q_{2}^{i n}}}{c_{2}}\right] \sqrt{k_{j}} c_{2}}{c_{3} \sqrt{v_{f}}\left(q_{1}^{i n}+\alpha q_{2}^{i n}\right)} \\
& +\frac{\log \left[q_{1}^{i n}+p \alpha q_{2}^{i n}\right] k_{j} q_{1}^{i n}}{2 c_{3} \alpha q_{2}^{i n}\left(q_{1}^{i n}+\alpha q_{2}^{i n}\right)}-\frac{\operatorname{ArcTanh}\left[\frac{\sqrt{c_{4}+4 p \alpha q_{2}^{i n}}}{c_{5}}\right] \sqrt{k_{j}} c_{5}}{c_{3} \sqrt{v_{f}}\left(q_{2}^{i n}-\alpha q_{2}^{i n}\right)}+\frac{\operatorname{ArcTanh}\left[\sqrt{\frac{c_{4}+4 p \alpha q_{2}^{i n}}{k_{j} v_{f}}}\right] k_{j}}{c_{3}\left(q_{2}^{i n}-\alpha q_{2}^{i n}\right)(-\alpha)} \\
& -\frac{\operatorname{ArcTanh}\left[\sqrt{\frac{c_{1}-4 p \alpha q_{2}^{i n}}{k_{j} v_{f}}}\right] k_{j} q_{1}^{i n}}{c_{3}\left(q_{1}^{i n}+\alpha q_{2}^{i n}\right) \alpha q_{2}^{i n}}+\frac{k_{j} \sqrt{\frac{c_{1}-4 p \alpha q_{2}^{i n}}{k_{j} v_{f}}}}{c_{3} \alpha q_{2}^{i n}}-\frac{k_{j} \sqrt{\frac{c_{4}+4 p \alpha q_{2}^{i n}}{k_{j} v_{f}}}}{c_{3} \alpha q_{2}^{i n}}
\end{aligned}
$$


(8). Time delay $t_{2 \rightarrow 1}^{g}$ when Lane 1 is under congestion flow and Lane 2 is under free flow.

$$
\begin{aligned}
& t_{2 \rightarrow 1}^{g}=E\left(T_{2 \rightarrow 1}^{g}\right)=\int_{0}^{L_{g}} T_{2 \rightarrow 1}^{g} P_{g}(r) d r=\int_{0}^{L_{g}} T_{2 \rightarrow 1}^{g} d F_{g}(r)=\left.T_{2 \rightarrow 1}^{g} F_{g}(r)\right|_{0} ^{L_{g}}-\int_{0}^{L_{g}} F_{g}(r) d T_{2 \rightarrow 1}^{g} \\
& =\left.T_{2 \rightarrow 1}^{g} F_{g}(r)\right|_{0} ^{L_{g}}-\int_{0}^{L_{g}} F_{g}(r)\left(\frac{1}{v_{2}^{g}(r)}-\frac{1}{v_{1}^{g}(r)}\right) d r \\
& =t_{2 \rightarrow 2}^{g} * F_{g}\left(L_{g}\right)-t_{1 \rightarrow 1}^{g} * \mathrm{p}-\frac{\log \left[q_{2}^{i n}-\alpha F_{g}\left(L_{g}\right) q_{2}^{i n}\right] k_{j}}{2 c_{3}(1-\alpha) \alpha q_{2}^{i n}}-\frac{\operatorname{ArcTanh}\left[\frac{\sqrt{c_{1}-4 \alpha F_{g}\left(L_{g}\right) q_{2}^{i n}}}{c_{2}}\right] \sqrt{k_{j}} c_{2}}{c_{3} \sqrt{v_{f}}\left(q_{1}^{i n}+\alpha q_{2}^{i n}\right)}
\end{aligned}
$$

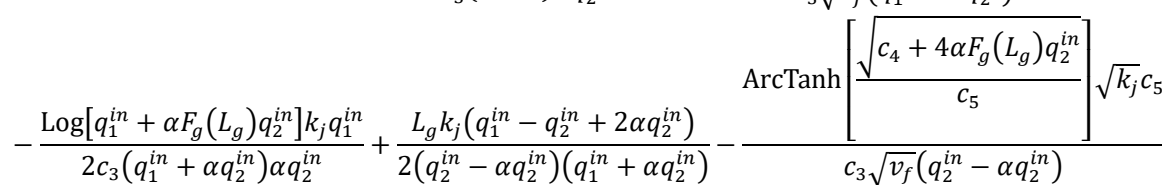

$$
\begin{aligned}
& +\frac{\operatorname{ArcTanh}\left[\sqrt{\frac{c_{4}+4 \alpha F_{g}\left(L_{g}\right) q_{2}^{i n}}{k_{j} v_{f}}}\right] k_{j} \quad \operatorname{ArcTanh}\left[\sqrt{\frac{c_{1}-4 \alpha F_{g}\left(L_{g}\right) q_{2}^{i n}}{k_{j} v_{f}}}\right] k_{j} q_{1}^{i n}}{c_{3}\left(q_{2}^{i n}-\alpha q_{2}^{i n}\right)(-\alpha)}-\frac{k_{j} \sqrt{\frac{c_{1}-4 \alpha F_{g}\left(L_{g}\right) q_{2}^{i n}}{k_{j} v_{f}}}}{c_{3} k_{j} v_{f}\left(q_{1}^{i n}+\alpha q_{2}^{i n}\right) q_{2}^{i n}}+\frac{c_{2} \alpha q_{2}^{i n}}{c_{n}} \\
& -\frac{k_{j} \sqrt{\frac{c_{4}+4 \alpha F_{g}\left(L_{g}\right) q_{2}^{i n}}{k_{j} v_{f}}}}{c_{3} \alpha q_{2}^{i n}}+\frac{\log \left[q_{2}^{i n}-p \alpha q_{2}^{i n}\right] k_{j} q_{2}^{i n}}{2 c_{3}\left(q_{2}^{i n}-\alpha q_{2}^{i n}\right) \alpha q_{2}^{i n}}+\frac{\operatorname{ArcTanh}\left[\frac{\sqrt{c_{1}-4 p \alpha q_{2}^{i n}}}{c_{2}}\right] \sqrt{k_{j}} c_{2}}{c_{3} \sqrt{v_{f}}\left(q_{1}^{i n}+\alpha q_{2}^{i n}\right)} \\
& +\frac{\log \left[q_{1}^{i n}+p \alpha q_{2}^{i n}\right] k_{j} q_{1}^{i n}}{2 c_{3} \alpha q_{2}^{i n}\left(q_{1}^{i n}+\alpha q_{2}^{i n}\right)}+\frac{\operatorname{ArcTanh}\left[\frac{\sqrt{c_{4}+4 p \alpha q_{2}^{i n}}}{c_{5}}\right] \sqrt{k_{j}} c_{5}}{c_{3} \sqrt{v_{f}}\left(q_{2}^{i n}-\alpha q_{2}^{i n}\right)}-\frac{\operatorname{ArcTanh}\left[\sqrt{\frac{c_{4}+4 p \alpha q_{2}^{i n}}{k_{j} v_{f}}}\right] k_{j}}{c_{3}\left(q_{2}^{i n}-\alpha q_{2}^{i n}\right)(-\alpha)} \\
& +\frac{\operatorname{ArcTanh}\left[\sqrt{\frac{c_{1}-4 p \alpha q_{2}^{i n}}{k_{j} v_{f}}}\right] k_{j} q_{1}^{i n}}{c_{3}\left(q_{1}^{i n}+\alpha q_{2}^{i n}\right) \alpha q_{2}^{i n}}-\frac{k_{j} \sqrt{\frac{c_{1}-4 p \alpha q_{2}^{i n}}{k_{j} v_{f}}}}{c_{3} \alpha q_{2}^{i n}}+\frac{k_{j} \sqrt{\frac{c_{4}+4 p \alpha q_{2}^{i n}}{k_{j} v_{f}}}}{c_{3} \alpha q_{2}^{i n}}
\end{aligned}
$$




\section{Appendix B}

The closed-form formulations for the time delay in the yellow zone will be given below. All the integration formulations are solved by Wolfram Mathematica 9.0.

\section{(1). Time delay $t_{1 \rightarrow 1}^{y}$ under Free flow condition}

$$
\begin{aligned}
t_{1 \rightarrow 1}^{y}=\int_{0}^{L_{y}}\left(\frac{d y}{v_{1}^{y}(\mathrm{y})}\right. & \left.-\frac{d y}{v_{1}^{g}(0)}\right) \\
& =\left\{2 \operatorname{ArcTan}\left(\frac{\sqrt{\mathrm{c}_{6}-4 F_{y}\left(L_{y}\right) \beta q_{2}^{y}(0)}}{\mathrm{c}_{7}}\right) \sqrt{k_{j}} \mathrm{c}_{7}\right. \\
& \left.-\left[2 \operatorname{ArcTanh}\left[\sqrt{\frac{\mathrm{c}_{6}-4 F_{y}\left(L_{y}\right) \beta q_{2}^{y}(0)}{k_{j} v_{f}}}\right]-L_{y} \mathrm{c}_{8}+\log \left[q_{1}^{y}(0)+F_{y}\left(L_{y}\right) \beta q_{2}^{y}(0)\right]\right] k_{j} \sqrt{v_{f}}\right\} \\
& /\left(2 \mathrm{c}_{8} \sqrt{v_{f}}\left(q_{1}^{y}(0)+\beta q_{2}^{y}(0)\right)\right) \\
& -\left\{2 \operatorname{ArcTan}\left(\frac{\sqrt{\mathrm{c}_{6}-4 \beta q_{2}^{y}(0)}}{\mathrm{c}_{7}}\right) \sqrt{k_{j} \mathrm{c}_{7}}\right. \\
& \left.-\left[2 \operatorname{ArcTanh}\left[\sqrt{\frac{\mathrm{c}_{6}-4 \beta q_{2}^{y}(0)}{k_{j} v_{f}}}\right]+\log \left[q_{1}^{y}(0)+\beta q_{2}^{y}(0)\right]\right] k_{j} \sqrt{v_{f}}\right\} \\
& /\left(2 \mathrm{c}_{8} \sqrt{v_{f}}\left(q_{1}^{y}(0)+\beta q_{2}^{y}(0)\right)\right)-\frac{L_{y}}{v_{1}^{g}(0)}
\end{aligned}
$$

Where:

$$
\begin{gathered}
\mathrm{c}_{6}=-4 q_{1}^{y}(0)+k_{j} v_{f} \\
\mathrm{c}_{7}=\sqrt{-4 q_{1}^{y}(0)+k_{j} v_{f}-4 \beta q_{2}^{y}(0)} \\
c_{8}=\frac{\left|v_{2}^{y \prime}-v_{1}^{y}\right| k_{1}^{y} \log [1-p]}{v_{2}^{y \prime}} \\
q_{1}^{y}(0)=q_{1}^{i n}+\alpha F_{g}\left(L_{g}\right) q_{2}^{i n} \\
q_{2}^{y}(0)=q_{2}^{i n}-\alpha F_{g}\left(L_{g}\right) q_{2}^{i n} \\
\beta=\frac{\alpha\left[1-F_{g}\left(L_{g}\right)\right]}{1-\alpha F_{g}\left(L_{g}\right)}
\end{gathered}
$$


(2). Time delay $t_{1 \rightarrow 1}^{y}$ under congestion flow condition:

$$
\begin{aligned}
t_{1 \rightarrow 1}^{y}=\int_{0}^{L_{y}}\left(\frac{d y}{v_{1}^{y}(y)}\right. & \left.-\frac{d y}{v_{1}^{g}(0)}\right) \\
& =\left\{2 \operatorname{ArcTan}\left(\frac{\sqrt{\mathrm{c}_{6}-4 F_{y}\left(L_{y}\right) \beta q_{2}^{y}(0)}}{\mathrm{c}_{7}}\right) \sqrt{k_{j} \mathrm{c}_{7}}\right. \\
& \left.-\left[2 \operatorname{ArcTanh}\left[\sqrt{\frac{\mathrm{c}_{6}-4 F_{y}\left(L_{y}\right) \beta q_{2}^{y}(0)}{k_{j} v_{f}}}\right]+L_{y} \mathrm{c}_{8}-\log \left[q_{1}^{y}(0)+F_{y}\left(L_{y}\right) \beta q_{2}^{y}(0)\right]\right] k_{j} \sqrt{v_{f}}\right\} \\
& /\left(2 \mathrm{c}_{8} \sqrt{v_{f}}\left(q_{1}^{y}(0)+\beta q_{2}^{y}(0)\right)\right) \\
& -\left\{2 \operatorname{ArcTan}\left(\frac{\sqrt{\mathrm{c}_{6}-4 \beta q_{2}^{y}(0)}}{\mathrm{c}_{7}}\right) \sqrt{k_{j} \mathrm{c}_{7}}\right. \\
& -\left[2 \operatorname{ArcTanh}\left[\sqrt{\frac{\mathrm{c}_{6}-4 \beta q_{2}^{y}(0)}{k_{j} v_{f}}}\right]-\log \left[q_{1}^{y}(0)+\beta q_{2}^{y}(0)\right] k_{j} \sqrt{v_{f}}\right\} \\
& /\left(2 \mathrm{c}_{8} \sqrt{v_{f}}\left(q_{1}^{y}(0)+\beta q_{2}^{y}(0)\right)\right)-\frac{L_{y}}{v_{1}^{g}(0)}
\end{aligned}
$$

(3). Time delay $t_{2 \rightarrow 1}^{y}$ when Lane 1 under free flow condition and lane 2 is under forward shock wave condition

$$
\begin{aligned}
& t_{2 \rightarrow 1}^{y}=E\left(T_{2 \rightarrow 1}^{y}\right)=\int_{0}^{L_{y}} T_{2 \rightarrow 1}^{y} P_{y}(r) d r=\int_{0}^{L_{y}} T_{2 \rightarrow 1}^{y} d F_{y}(r)=\left.T_{2 \rightarrow 1}^{y} F_{y}(r)\right|_{0} ^{L_{y}}-\int_{0}^{L_{y}} F_{y}(r) d T_{2 \rightarrow 1}^{y} \\
& =\left.T_{2 \rightarrow 1}^{y} F_{y}(r)\right|_{0} ^{L_{y}}-\int_{0}^{L_{y}} F_{y}(r)\left(\frac{1}{v_{2}^{y \prime}}-\frac{1}{v_{1}^{y}(r)}\right) d r \\
& =\left(\frac{L_{y}}{v_{2}^{y \prime}}-\frac{L_{y}}{v_{2}^{i n}}\right) * F_{y}\left(L_{y}\right)-t_{1 \rightarrow 1}^{y}+\frac{F_{y}\left(L_{y}\right)-1}{c_{8} v_{2}^{y \prime}} \\
& +\left\{2 \beta \operatorname{ArcTan}\left(\frac{\sqrt{\mathrm{c}_{6}-4 F_{y}\left(L_{y}\right) \beta q_{2}^{y}(0)}}{\mathrm{c}_{7}}\right) \sqrt{k_{j} \mathrm{c}_{7}} q_{2}^{y}(0)\right. \\
& -k_{j} \sqrt{v_{f}} \beta q_{2}^{y}(0)\left(2 \sqrt{\frac{c_{6}-4 F_{y}\left(L_{y}\right) \beta q_{2}^{y}(0)}{k_{j} v_{f}}}-L_{y} c_{8}\right) \\
& -k_{j} \sqrt{v_{f}} q_{1}^{y}(0)\left(2 \sqrt{\frac{\mathrm{c}_{6}-4 F_{y}\left(L_{y}\right) \beta q_{2}^{y}(0)}{k_{j} v_{f}}}-2 \operatorname{ArcTanh}\left[\sqrt{\frac{\mathrm{c}_{6}-4 F_{y}\left(L_{y}\right) \beta q_{2}^{y}(0)}{k_{j} v_{f}}}\right]\right. \\
& \left.\left.-\log \left[q_{1}^{y}(0)+F_{y}\left(L_{y}\right) \beta q_{2}^{y}(0)\right]\right)\right\} /\left(2 c_{8} \sqrt{v_{f}}\left(q_{1}^{y}(0)+\beta q_{2}^{y}(0)\right) q_{2}^{y}(0)\right)
\end{aligned}
$$

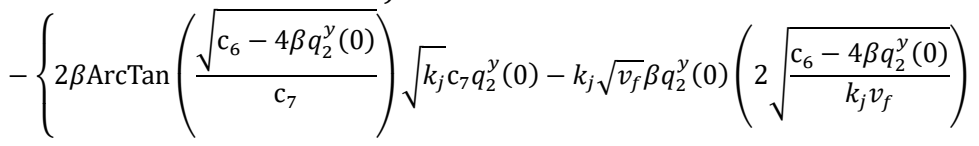

$$
\begin{aligned}
& \left.-k_{j} \sqrt{v_{f}} q_{1}^{y}(0)\left(2 \sqrt{\frac{c_{6}-4 \beta q_{2}^{y}(0)}{k_{j} v_{f}}}-2 \operatorname{ArcTanh}\left[\sqrt{\frac{c_{6}-4 \beta q_{2}^{y}(0)}{k_{j} v_{f}}}\right]-\log \left[q_{1}^{y}(0)+\beta q_{2}^{y}(0)\right]\right)\right\} \\
& /\left(2 \mathrm{c}_{8} \sqrt{v_{f}}\left(q_{1}^{y}(0)+\beta q_{2}^{y}(0)\right) q_{2}^{y}(0)\right)
\end{aligned}
$$


(4). Time delay $t_{2 \rightarrow 1}^{y}$ when Lane 1 under congestion flow condition and lane 2 is under forward shock wave condition

$$
\begin{aligned}
t_{2 \rightarrow 1}^{y}=E\left(T_{2 \rightarrow 1}^{y}\right)=\int_{0}^{L_{y}} & T_{2 \rightarrow 1}^{y} P_{y}(r) d r=\int_{0}^{L_{y}} T_{2 \rightarrow 1}^{y} d F_{y}(r)=\left.T_{2 \rightarrow 1}^{y} F_{y}(r)\right|_{0} ^{L_{y}}-\int_{0}^{L_{y}} F_{y}(r) d T_{2 \rightarrow 1}^{y} \\
& =\left.T_{2 \rightarrow 1}^{y} F_{y}(r)\right|_{0} ^{L_{y}}-\int_{0}^{L_{y}} F_{y}(r)\left(\frac{1}{v_{2}^{y \prime}}-\frac{1}{v_{1}^{y}(r)}\right) d r \\
& =\left(\frac{L_{y}}{v_{2}^{y \prime}}-\frac{L_{y}}{v_{2}^{i n}}\right) * F_{y}\left(L_{y}\right)-t_{1 \rightarrow 1}^{y}+\frac{F_{y}\left(L_{y}\right)-1}{c_{8} v_{2}^{y \prime}} \\
& +\left\{-2 \beta \operatorname{ArcTan}\left(\frac{\sqrt{c_{6}-4 F_{y}\left(L_{y}\right) \beta q_{2}^{y}(0)}}{c_{7}}\right) \sqrt{k_{j} c_{7}} q_{2}^{y}(0)\right. \\
& +k_{j} \sqrt{v_{f}} \beta q_{2}^{y}(0)\left(2 \sqrt{\frac{c_{6}-4 F_{y}\left(L_{y}\right) \beta q_{2}^{y}(0)}{k_{j} v_{f}}}+L_{y} c_{8}\right) \\
& +k_{j} \sqrt{v_{f}} q_{1}^{y}(0)\left(2 \sqrt{\frac{c_{6}-4 F_{y}\left(L_{y}\right) \beta q_{2}^{y}(0)}{k_{j} v_{f}}}-2 \operatorname{ArcTanh}\left[\sqrt{\frac{c_{6}-4 F_{y}\left(L_{y}\right) \beta q_{2}^{y}(0)}{k_{j} v_{f}}}\right]\right. \\
& \left.+\log \left[q_{1}^{y}(0)+F_{y}\left(L_{y}\right) \beta q_{2}^{y}(0)\right]\right) /\left(2 c_{8} \sqrt{v_{f}}\left(q_{1}^{y}(0)+\beta q_{2}^{y}(0)\right) q_{2}^{y}(0)\right) \\
& -\left\{-2 \beta \operatorname{ArcTan}\left(\frac{\sqrt{c_{6}-4 \beta q_{2}^{y}(0)}}{c_{7}}\right) \sqrt{k_{j}} c_{7} q_{2}^{y}(0)+k_{j} \sqrt{v_{f} \beta q_{2}^{y}(0)}\left(2 \sqrt{\frac{c_{6}-4 \beta q_{2}^{y}(0)}{k_{j} v_{f}}}\right)\right. \\
& \left.+k_{j} \sqrt{v_{f}} q_{1}^{y}(0)\left(2 \sqrt{\frac{c_{6}-4 \beta q_{2}^{y}(0)}{k_{j} v_{f}}}-2 \operatorname{ArcTanh}\left[\sqrt{\frac{c_{6}-4 \beta q_{2}^{y}(0)}{k_{j} v_{f}}}\right]+\log \left[q_{1}^{y}(0)+\beta q_{2}^{y}(0)\right]\right)\right\} \\
& /\left(2 c_{8} \sqrt{v_{f}}\left(q_{1}^{y}(0)+\beta q_{2}^{y}(0)\right) q_{2}^{y}(0)\right)
\end{aligned}
$$

(5). Time delay $t_{2 \rightarrow 1}^{y}$ when Lane 1 under free flow condition and lane 2 is under backward shock wave condition

$$
\begin{aligned}
t_{2 \rightarrow 1}^{y}=E\left(T_{2 \rightarrow 1}^{y}\right)=\int_{0}^{L_{y}} & T_{2 \rightarrow 1}^{y} P_{y}(r) d r=\int_{0}^{L_{y}} T_{2 \rightarrow 1}^{y} d F_{y}(r)=\left.T_{2 \rightarrow 1}^{y} F_{y}(r)\right|_{0} ^{L_{y}}-\int_{0}^{L_{y}} F_{y}(r) d T_{2 \rightarrow 1}^{y} \\
& =\left.T_{2 \rightarrow 1}^{y} F_{y}(r)\right|_{0} ^{L_{y}}-\int_{0}^{L_{y}} F_{y}(r)\left(\frac{1}{v_{2}^{y \prime}}-\frac{1}{v_{1}^{y}(r)}\right) d r \\
& =\left(\frac{L_{y}}{v_{2}^{y \prime}}-\frac{L_{y}}{v_{2}^{i n}}\right) * F_{y}\left(L_{y}\right)-t_{1 \rightarrow 1}^{y}+\frac{F_{y}\left(L_{y}\right)-1}{\mathrm{c}_{8} v_{2}^{y_{\prime}}} \\
& +\left\{2 \beta \operatorname{ArcTan}\left(\frac{\sqrt{\mathrm{c}_{6}-4 F_{y}\left(L_{y}\right) \beta q_{2}^{y}(0)}}{\mathrm{c}_{7}}\right) \sqrt{k_{j} \mathrm{c}_{7} q_{2}^{y}(0)}\right. \\
& -k_{j} \sqrt{v_{f}} \beta q_{2}^{y}(0)\left(2 \sqrt{\frac{\mathrm{c}_{6}-4 F_{y}\left(L_{y}\right) \beta q_{2}^{y}(0)}{k_{j} v_{f}}}-L_{y} \mathrm{c}_{8}\right) \\
& -k_{j} \sqrt{v_{f}} q_{1}^{y}(0)\left(2 \sqrt{\frac{\mathrm{c}_{6}-4 F_{y}\left(L_{y}\right) \beta q_{2}^{y}(0)}{k_{j} v_{f}}}-2 \operatorname{ArcTanh}\left[\sqrt{\frac{\mathrm{c}_{6}-4 F_{y}\left(L_{y}\right) \beta q_{2}^{y}(0)}{k_{j} v_{f}}}\right]\right. \\
& \left.-\log \left[q_{1}^{y}(0)+F_{y}\left(L_{y}\right) \beta q_{2}^{y}(0)\right]\right) /\left(2 c_{8} \sqrt{v_{f}}\left(q_{1}^{y}(0)+\beta q_{2}^{y}(0)\right) q_{2}^{y}(0)\right) \\
& -\left\{2 \beta \operatorname{ArcTan}\left(\frac{\sqrt{c_{6}-4 \beta q_{2}^{y}(0)}}{\mathrm{c}_{7}}\right) \sqrt{k_{j} c_{7} q_{2}^{y}(0)-k_{j} \sqrt{v_{f}} \beta q_{2}^{y}(0)}\left(2 \sqrt{\frac{\mathrm{c}_{6}-4 \beta q_{2}^{y}(0)}{k_{j} v_{f}}}\right)\right. \\
& \left.-k_{j} \sqrt{v_{f}} q_{1}^{y}(0)\left(2 \sqrt{\frac{\mathrm{c}_{6}-4 \beta q_{2}^{y}(0)}{k_{j} v_{f}}}-2 \operatorname{ArcTanh}\left[\sqrt{\frac{\mathrm{c}_{6}-4 \beta q_{2}^{y}(0)}{k_{j} v_{f}}}\right]-\log \left[q_{1}^{y}(0)+\beta q_{2}^{y}(0)\right]\right)\right\} \\
& /\left(2 c_{8} \sqrt{v_{f}}\left(q_{1}^{y}(0)+\beta q_{2}^{y}(0)\right) q_{2}^{y}(0)\right)+T_{2}^{y b}
\end{aligned}
$$

Where, $\mathrm{T}_{2}^{y b}$ is based on Equation (44). 
(6). Time delay $t_{2 \rightarrow 1}^{y}$ when Lane 1 under congestion flow condition and lane 2 is under backward shock wave condition

$$
\begin{aligned}
t_{2 \rightarrow 1}^{y}=E\left(T_{2 \rightarrow 1}^{y}\right)=\int_{0}^{L_{y}} & T_{2 \rightarrow 1}^{y} P_{y}(r) d r=\int_{0}^{L_{y}} T_{2 \rightarrow 1}^{y} d F_{y}(r)=\left.T_{2 \rightarrow 1}^{y} F_{y}(r)\right|_{0} ^{L_{y}}-\int_{0}^{L_{y}} F_{y}(r) d T_{2 \rightarrow 1}^{y} \\
& =\left.T_{2 \rightarrow 1}^{y} F_{y}(r)\right|_{0} ^{L_{y}}-\int_{0}^{L_{y}} F_{y}(r)\left(\frac{1}{v_{2}^{y \prime}}-\frac{1}{v_{1}^{y}(r)}\right) d r \\
& =\left(\frac{L_{y}}{v_{2}^{y \prime}}-\frac{L_{y}}{v_{2}^{i n}}\right) * F_{y}\left(L_{y}\right)-t_{1 \rightarrow 1}^{y}+\frac{F_{y}\left(L_{y}\right)-1}{c_{8} v_{2}^{y \prime}} \\
& +\left\{-2 \beta \operatorname{ArcTan}\left(\frac{\sqrt{c_{6}-4 F_{y}\left(L_{y}\right) \beta q_{2}^{y}(0)}}{c_{7}}\right) \sqrt{k_{j} c_{7}} q_{2}^{y}(0)\right. \\
& +k_{j} \sqrt{v_{f}} \beta q_{2}^{y}(0)\left(2 \sqrt{\frac{c_{6}-4 F_{y}\left(L_{y}\right) \beta q_{2}^{y}(0)}{k_{j} v_{f}}}+L_{y} c_{8}\right) \\
& +k_{j} \sqrt{v_{f}} q_{1}^{y}(0)\left(2 \sqrt{\frac{c_{6}-4 F_{y}\left(L_{y}\right) \beta q_{2}^{y}(0)}{k_{j} v_{f}}}-2 \operatorname{ArcTanh}\left[\sqrt{\frac{c_{6}-4 F_{y}\left(L_{y}\right) \beta q_{2}^{y}(0)}{k_{j} v_{f}}}\right]\right. \\
& \left.+\log \left[q_{1}^{y}(0)+F_{y}\left(L_{y}\right) \beta q_{2}^{y}(0)\right]\right) /\left(2 c_{8} \sqrt{v_{f}}\left(q_{1}^{y}(0)+\beta q_{2}^{y}(0)\right) q_{2}^{y}(0)\right) \\
& -\left\{-2 \beta \operatorname{ArcTan}\left(\frac{\sqrt{c_{6}-4 \beta q_{2}^{y}(0)}}{\mathrm{c}_{7}}\right) \sqrt{k_{j}} c_{7} q_{2}^{y}(0)+k_{j} \sqrt{v_{f} \beta q_{2}^{y}(0)}\left(2 \sqrt{\frac{c_{6}-4 \beta q_{2}^{y}(0)}{k_{j} v_{f}}}\right)\right. \\
& \left.+k_{j} \sqrt{v_{f}} q_{1}^{y}(0)\left(2 \sqrt{\frac{c_{6}-4 \beta q_{2}^{y}(0)}{k_{j} v_{f}}}-2 \operatorname{ArcTanh}\left[\sqrt{\frac{c_{6}-4 \beta q_{2}^{y}(0)}{k_{j} v_{f}}}\right]+\log \left[q_{1}^{y}(0)+\beta q_{2}^{y}(0)\right]\right)\right\} \\
& /\left(2 c_{8} \sqrt{v_{f}}\left(q_{1}^{y}(0)+\beta q_{2}^{y}(0)\right) q_{2}^{y}(0)\right)+T_{2}^{y b}
\end{aligned}
$$

